\title{
Conditioning the Estimating Ultimate Recovery of Shale Wells to Reservoir and Completion Parameters
}

\author{
Maher Jasim Alabboodi
}

Follow this and additional works at: https://researchrepository.wvu.edu/etd

\section{Recommended Citation}

Alabboodi, Maher Jasim, "Conditioning the Estimating Ultimate Recovery of Shale Wells to Reservoir and Completion Parameters" (2016). Graduate Theses, Dissertations, and Problem Reports. 5044.

https://researchrepository.wvu.edu/etd/5044

This Thesis is protected by copyright and/or related rights. It has been brought to you by the The Research Repository @ WVU with permission from the rights-holder(s). You are free to use this Thesis in any way that is permitted by the copyright and related rights legislation that applies to your use. For other uses you must obtain permission from the rights-holder(s) directly, unless additional rights are indicated by a Creative Commons license in the record and/ or on the work itself. This Thesis has been accepted for inclusion in WVU Graduate Theses, Dissertations, and Problem Reports collection by an authorized administrator of The Research Repository @ WVU. For more information, please contact researchrepository@mail.wvu.edu. 
Conditioning the Estimating Ultimate Recovery of Shale Wells to Reservoir and Completion Parameters

\author{
Maher Jasim Alabboodi \\ Thesis submitted to the \\ Benjamin M. Statler College of Engineering and Mineral Resources \\ at West Virginia University
}

In partial fulfillment of the requirements for the degree of

Master of Science

in

Petroleum and Natural Gas Engineering

Shahab Mohaghegh, PhD, Chair

Samuel Ameri, Prof.

Dengliang Gao, Ph.D.

Department of Petroleum and Natural Gas Engineering

Morgantown, West Virginia

2016

Keywords: Decline Curves Analysis, Estimated Ultimate Recovery, Artificial Neural

Network, Pattern Recognition.

Copyright 2016 Maher Jasim Alabboodi 


\section{Abstract \\ Conditioning the Estimating Ultimate Recovery of Shale Wells to Reservoir and Completion Parameters}

\section{Maher Jasim Alabboodi}

In the last years, gas production from shale has increased significantly in the United States. Therefore, many studies have been focused on shale formation in different areas such as fracturing, reservoir simulation, forecasting and so on. Forecasting production or estimating ultimate recovery (EUR) is considered to be one of the most important items in the production development planning. The certainty in EUR calculation is questionable because there are different parameters that impact production and consequently the EUR such as rock properties and well completion design.

Different methods to calculate EUR have been used in the industry. Traditionally, the decline curve analysis method by Arps (1945) was considered to be the best common tool for estimating ultimate recovery (EUR) and reserves. However, the Arps' equations over estimate of reserves when they are applied to unconventional reservoirs (extremely low permeability formation). The reason is that Arps' equations only work for Boundary Dominated Flow (BDF) decline. On the other hand, many research papers show that the production from the unconventional tight reservoirs is distinguished by an extended period of late transient flow, until reaching the boundary-dominated flow. To overcome these problems and improve the unconventional reservoir's production forecast, researchers have developed new empirical methods which are being implemented in all flow regimes.

These new and traditional methods have been applied in this research to calculate the EUR for more than 200 shale wells. The results of EUR will be subjected to study and condition with rock properties, well characteristics and completion's design parameters. The porosity, total organic carbon, net thickness and water saturation are the main rock properties that are considered in this research. Furthermore, the impact of different well design configurations (for instance, well trajectories, completion and hydraulic fracturing variable) on EUR will be inspected this study. In addition, it will be determined from this research whether reservoir or completion parameters have the most impact on EUR. This study will provide the natural gas professionals insight and clarification regarding the effects of rock properties and well design configurations on estimating the ultimate recovery for gas shale. 


\section{Dedication}

To my Father and Mother 


\section{Acknowledgements}

Firstly, I would like to express my sincere gratitude to my advisor, Dr. Shahab Mohaghegh, for his excellent guidance, caring, patience, and providing me with excellent data for doing my research. In addition, I express my gratitude to my committee members, Professor Samuel Ameri and Dr. Dengliang Gao, for academic support.

In addition, I would like to express my deepest appreciation to my sponsor, The Higher Committee for Education Development in Iraq, for giving me the opportunity to complete my graduate studies and for providing me with financial support during my study.

To all relatives, friends and others who in one way or another shared their support and help, thank you. I owe a deep sense of gratitude to my special friend, Mr. Robert Jones, who stood by me always in good and bad days in Morgantown.

I would also like to thank my family who were supporting me and encouraging me with their best wishes. They were always there cheering me up and stood by me through the good and bad days. Last but not least, I am extremely thankful to my God who gave me everything to do this project. 


\section{Table of Contents}

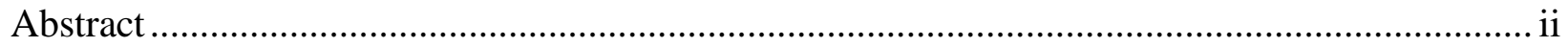

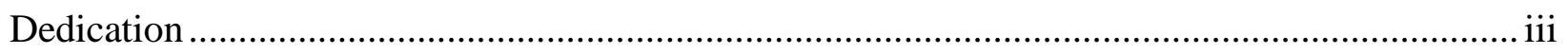

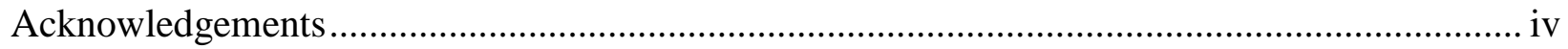

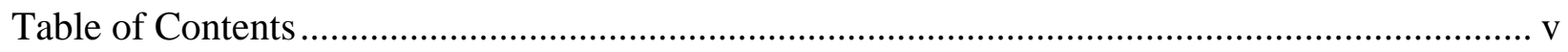

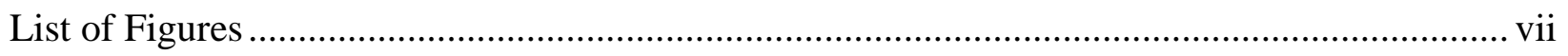

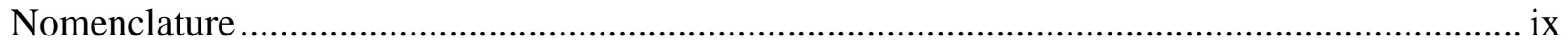

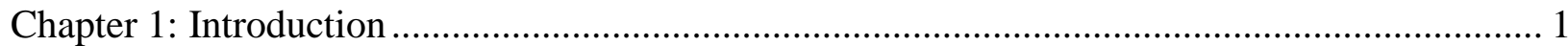

1.1 Introduction to the estimated ultimate recovery................................................. 1

1.2 Unconventional Gas Shale Resources in the United States ....................................... 2

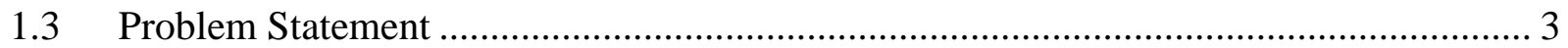

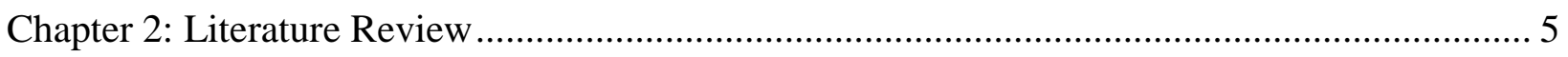

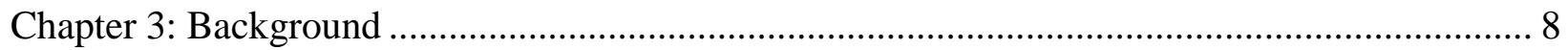

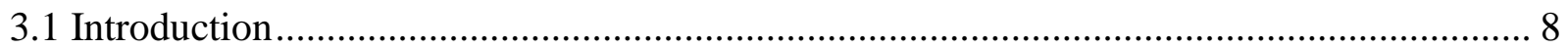

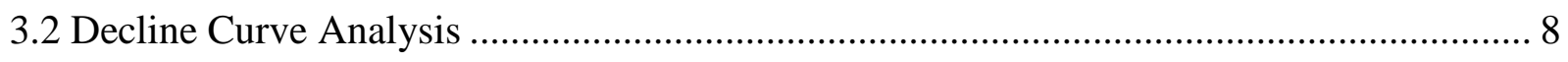

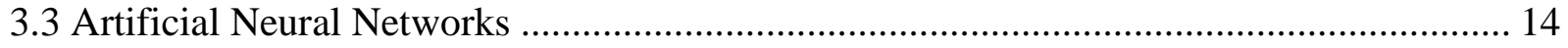

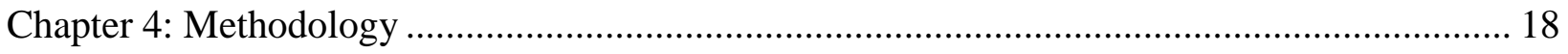

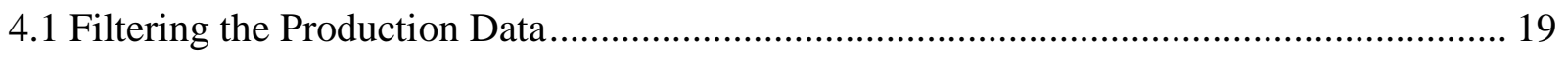

4.2. Calculating the Estimated Ultimate Recovery (EUR) ............................................. 20

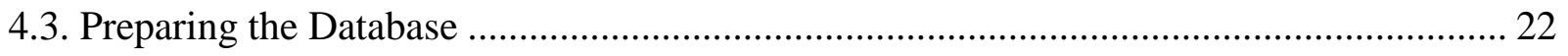

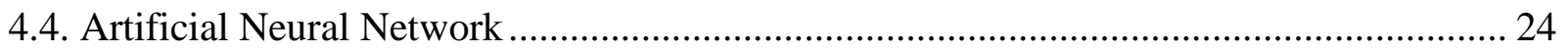

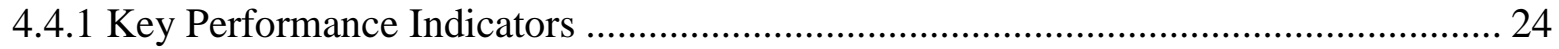

4.4.2 Data Partitioning and Neural Network Training ............................................... 26

4.4.3 Fuzzy Pattern Recognition analyses ................................................................. 31

4.4.4 Generating Type Curve ............................................................................. 34

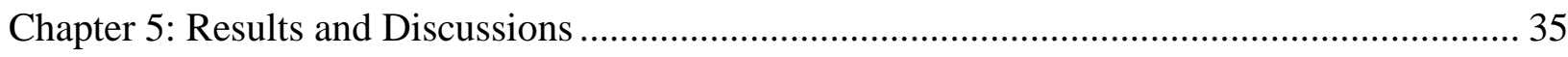

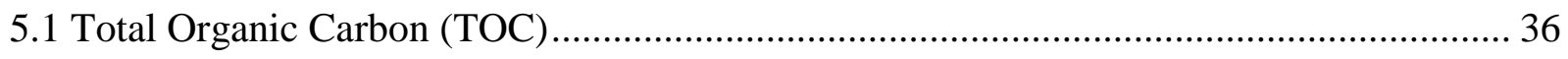

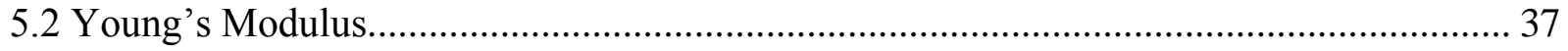

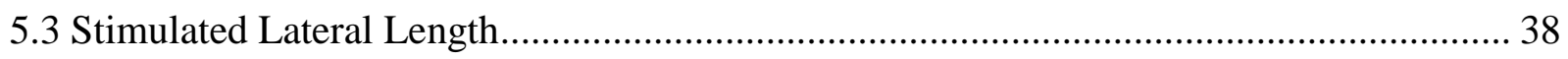

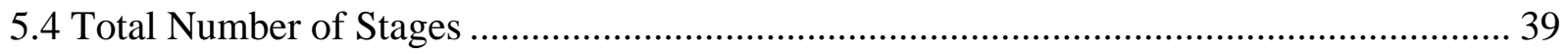




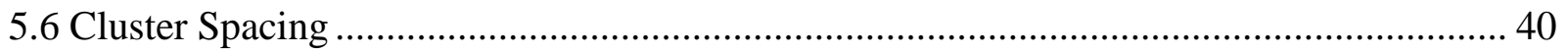

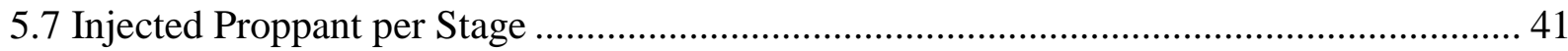

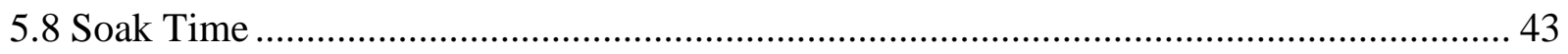

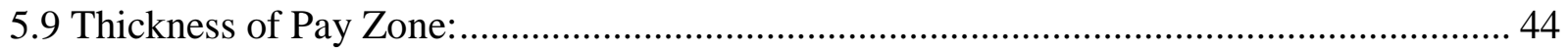

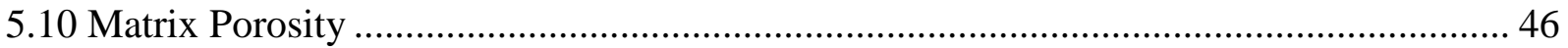

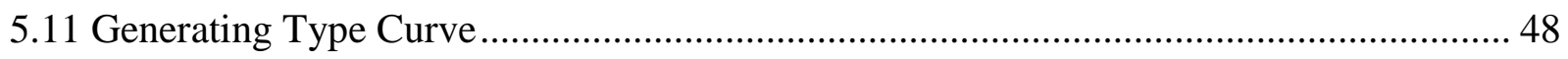

5.11.1 EUR as a Function of Measured Depth and Porosity in Type Curve ....................... 48

5.11.2 EUR as a Function of True Vertical Depth and Lateral Length in Type Curve ........ 49

5.11.3 EUR as a Function of Lateral Length and Number of Stages in Type Curve ........... 50

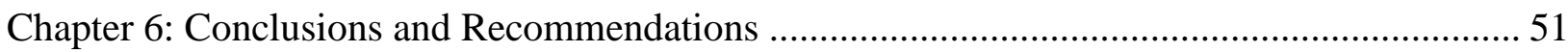

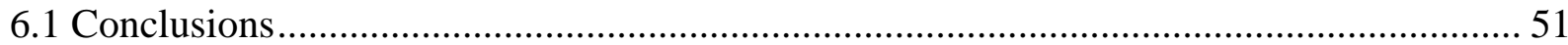

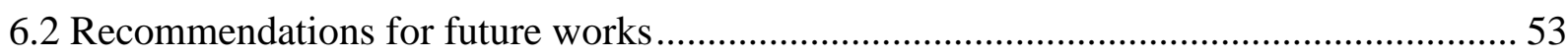

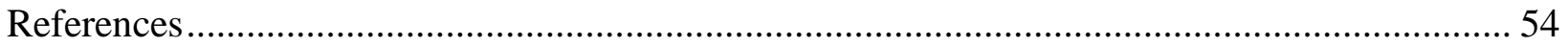

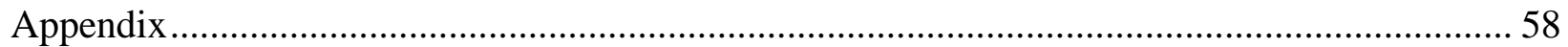

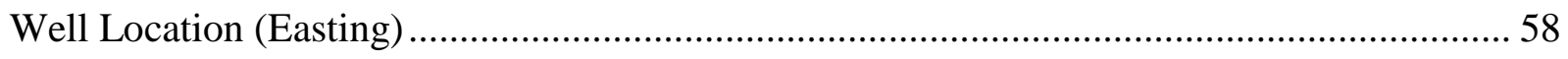

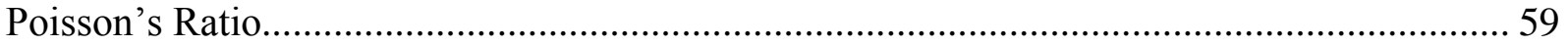

Total Organic Carbon (Well Quality Analysis) ........................................................................ 60

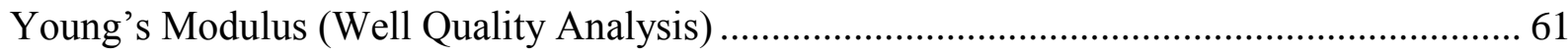

Stimulated Lateral Length (Well Quality Analysis) …………………………………........... 62

Number of Stages (Well Quality Analysis) ............................................................................. 63

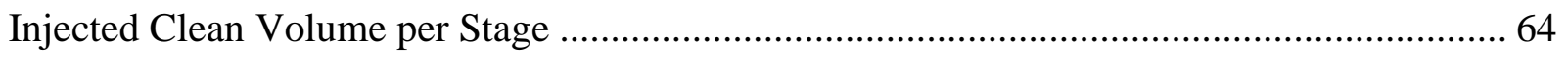

EUR as a Function of Lateral Length and Cluster Spacing in Type Curve:.............................. 65

EUR as a Function of Lateral Length and Clean Volume in Type Curve: ................................. 66

EUR as a Function of Lateral Length and Net Thickness in Type Curve: ............................... 67 


\section{List of Figures}

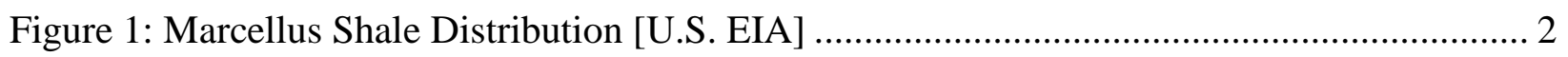

Figure 2: Reservoir and completion parameters …………................................................... 4

Figure 3: Applying Arps' equations in conventional reservoir [Courtesy of Reference 1] ............ 9

Figure 4: Applying Arps' equations in unconventional reservoir [Courtesy of Reference 1] ...... 10

Figure 5: Stretched Exponential Decline Model Behavior [Courtesy of Reference 2] ................ 13

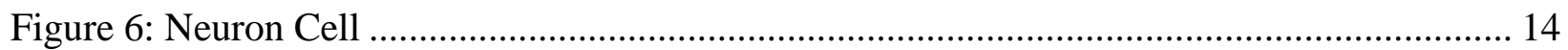

Figure 7: Mathematical structure for neuron of Artificial Neural Networks................................. 15

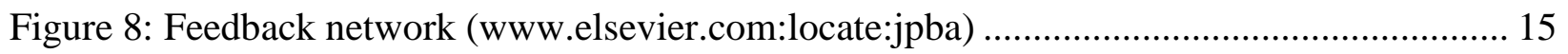

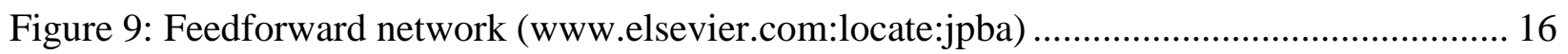

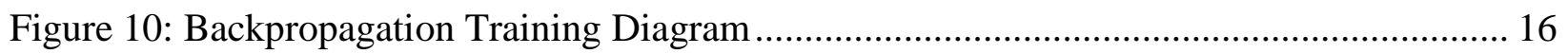

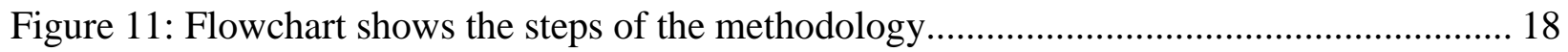

Figure 12: Applying the Four Models on Field Data- Well \#10030-1 ………………................ 21

Figure 13: Native and Design Parameters ………………..................................................... 23

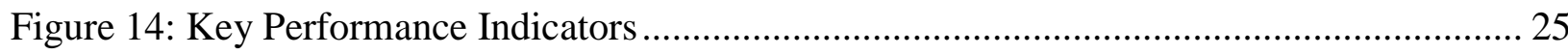

Figure 15: Design for the Back-Propagation Neural Network ……………………………........ 27

Figure 16: The training process of the Neural Network (PLE MODEL) ……………………..... 28

Figure 17: Backpropagation result for the trained NN-All Cases- Scatter Plot............................ 29

Figure 18: Backpropagation result for the trained NN-All Cases- Scatter Plot............................ 29

Figure 19: The impact of well location (Easting) on EUR for the models TED, PLE, SEPD and

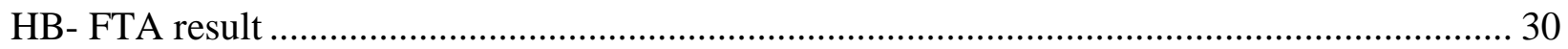

Figure 20: Backpropagation result for the trained NN-Training Data -Scatter Plot...................... 30

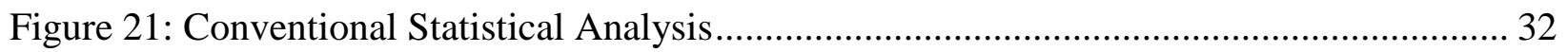

Figure 22: Fuzzy Trend Recognition- 10 years PLE EUR vs. \% Porosity ..................................... 33

Figure 23: Well Quality Analysis based on porosity and 10 years PLE EUR............................... 34

Figure 24: The impact of TOC on EUR for the models TED, PLE, SEPD and HB- FTA result. 36

Figure 25: The impact of Young's Modulus on EUR for the models TED, PLE, SEPD and HB-

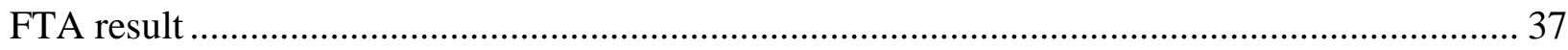

Figure 26: The impact of Stimulated Lateral Length on EUR for the models TED, PLE, SEPD

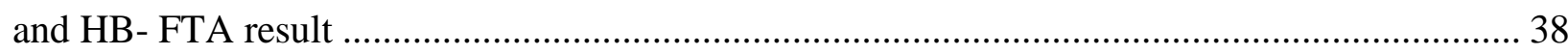

Figure 27: The impact of Number of Stages on EUR for the models TED, PLE, SEPD and HB-

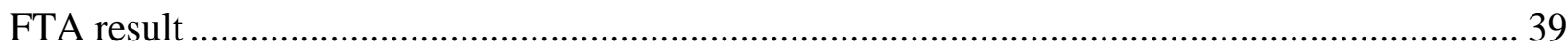

Figure 28: The impact of Cluster Spacing on EUR for the models TED, PLE, SEPD and HB-

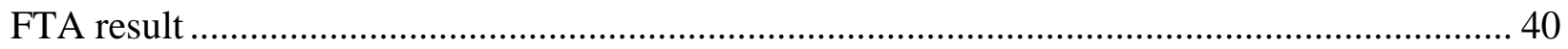

Figure 29: Well Quality Analysis of Cluster Spacing for models TED, PLE, SEPD and HB ..... 41

Figure 30: The impact of Injected Proppant per stage on EUR for the models TED, PLE, SEPD

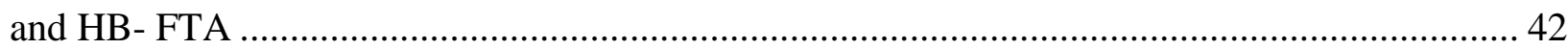

Figure 31: Well Quality Analysis of Injected Proppant per Stage for EUR models TED, PLE,

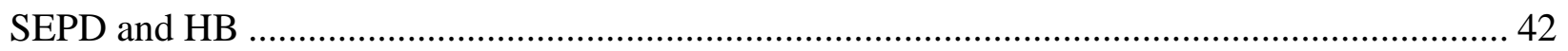


Figure 32: The impact of Soak Time on EUR for the models TED, PLE, SEPD and HB- FTA result.

Figure 33: Well Quality Analysis of Soak Time for EUR models TED, PLE, SEPD and HB .... 44 Figure 34: The impact of Net Thickness on EUR for the models TED, PLE, SEPD and HB- FTA result.

Figure 35: Well Quality Analysis of Net Thickness for EUR models TED, PLE, SEPD and HB45 Figure 36: The impact of Matrix Porosity on EUR for the models TED, PLE, SEPD and HBFTA result 46

Figure 37: Well Quality Analysis of Matrix Porosity for EUR models TED, PLE, SEPD and HB

.

Figure 38: EUR as a Function of Measured Depth and Porosity in Type Curve........................ 48

Figure 39: EUR as a Function of True Vertical Depth and Lateral Length in Type Curve.......... 49

Figure 40: EUR as a Function of Lateral Length and Number of Stages in Type Curve............ 50

Figure 41: The Average Influence Degree of Native and Design Parameters on EUR.............. 51

Figure 42: The impact of well location (Easting) on EUR for the models TED, PLE, SEPD and

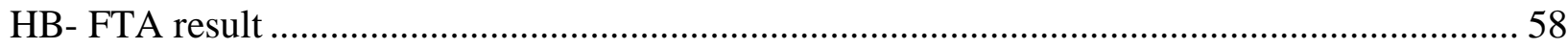

Figure 43: The impact of Poisson's Ratio on EUR for the models TED, PLE, SEPD and HB-

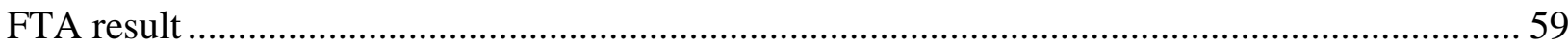

Figure 44: Well Quality Analysis of Total Organic Carbon for EUR models TED, PLE, SEPD

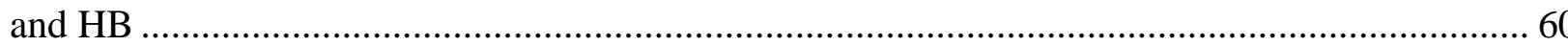

Figure 45: Well Quality Analysis of Young's Modulus for EUR models TED, PLE, SEPD and

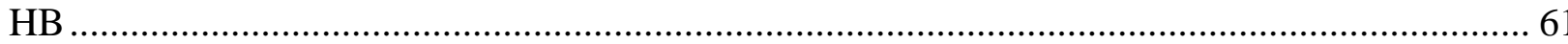

Figure 46: Well Quality Analysis of Lateral Length for EUR models TED, PLE, SEPD and HB

Figure 47: Well Quality Analysis of Number of Stages for EUR models TED, PLE, SEPD and HB

Figure 48: Well Quality Analysis of Injected Clean Volume per Stage for EUR models TED, PLE, SEPD and HB 64

Figure 49: EUR as a Function of Lateral Length and Cluster Spacing in Type Curve ............... 65

Figure 50: EUR as a Function of Lateral Length and Clean Volume in Type Curve.................. 66

Figure 51: EUR as a Function of Lateral Length and Net Thickness in Type Curve.................. 67 


\section{Nomenclature}

EUR: Estimated Ultimate Recovery

DCA: Decline Curve Analysis

BDF: Boundary-Dominated Flow

PLE: Power Law Exponential Method

SEPD: Stretched Exponential Production Decline Method

HB: Hyperbolic Method

TED: Tail End Exponential Method

ANNs: Artificial Neural Networks

KPI: Key Performance Indicator

FPR: Fuzzy Pattern Recognition

FTA: Fuzzy Trend Analysis

WQA: Well Quality Analysis

TOC: Total Organic Carbon 


\section{Chapter 1: Introduction}

\subsection{Introduction to the estimated ultimate recovery}

For decades, oil and natural gas energy has continued to be the main energy resource around the world. Petroleum reservoirs are defined based on the rocks characteristics to conventional and unconventional reservoirs. In general, Conventional reservoirs have high permeability and mostly produce naturally with high flow rate at the initial reservoir pressure. In contrast, unconventional reservoirs have very low permeability and porosity which typically need stimulation job to increase the permeability and producing. However, recently, advances in horizontal drilling technology and the development of hydraulic fracturing technology have improved access to unconventional reservoirs deposits and make these reservoirs the main gas resources in United States.

In consequence, evaluating the amount of oil and natural gas recoverable is very important for both economic and operational purposes. Many methods have been developed to estimate the oil and natural gas recoverable in the reservoirs. One of most used technique in the industry is the decline curve analysis which used to calculate the estimated ultimate recovery (EUR). Decline curve analysis is defined as a graphical technique used for analyzing declining production rates and forecasting future performance of oil and gas wells. Different equations derived from the main concept of decline curve are developed to achieve this purpose. The accuracy in this technique depends on the equation that used to calculate the EUR.

Traditionally, Arps' equations have been used to calculate the EUR for conventional reservoir when exhibit boundary-dominated flow regime. However, these equations show unreasonable result when applied in unconventional reservoir due to low permeability that causes long periods of transient flow. To overcome this problem, several techniques have been developed to calculate the EUR in unconventional reservoirs such as Power Law Exponential by Ilk et al. (2008) and Stretched Exponential Decline by Valko (2009). In addition, in 2010, Duong has developed a new empirical model based on long-term liner flow in tight gas reservoir. Duong considers his approach easy and simple to use for predicting the future rate and EUR.

Different parameters such as reservoir characteristic or well completion design impact in the flow rate of the production from the reservoirs and consequently affect the estimated ultimate recovery for these reservoirs. Study the effects of these parameters on the RUR results provide an understanding of which completion design or drilling spot should be applied. 


\subsection{Unconventional Gas Shale Resources in the United States}

In recent past, natural gas sealed in tight, impermeable shale which called unconventional reservoirs was considered uneconomical source to produce. However, using new technologies in drilling and reservoir stimulation techniques have changed these thoughts by significantly increasing the gas production from the uneconomical reservoirs. In United States, the most prompt development of the unconventional reservoirs has been in The Barnett Shale formation in Texas. Though, Marcellus Shale formation, which is in the northeastern part, considered the largest unconventional gas resource in the United States.

Unconventional gas shales describe as fine grained, sedimentary rocks, organic rich. Therefore, both the sources and the reservoirs of the natural gas found in the shales formations. In the shales formation, gas occupies pore spaces while organic matter adsorbs gas on its surface. On the other hand, in conventional reservoir, oil and natural gas generate in porous sandstone and carbonate reservoirs. Then, the oil and natural gas are migrated upward from its organic source until an impermeable cap-rock trapped it in the reservoir.

Recently, the Marcellus Shale formations stand out as a significant new and major source of natural gas production in the United States. It extends from Ohio through West Virginia, western Maryland, and into Pennsylvania and New York. Figure (1) shows the distribution of Marcellus Shale formations in the United States

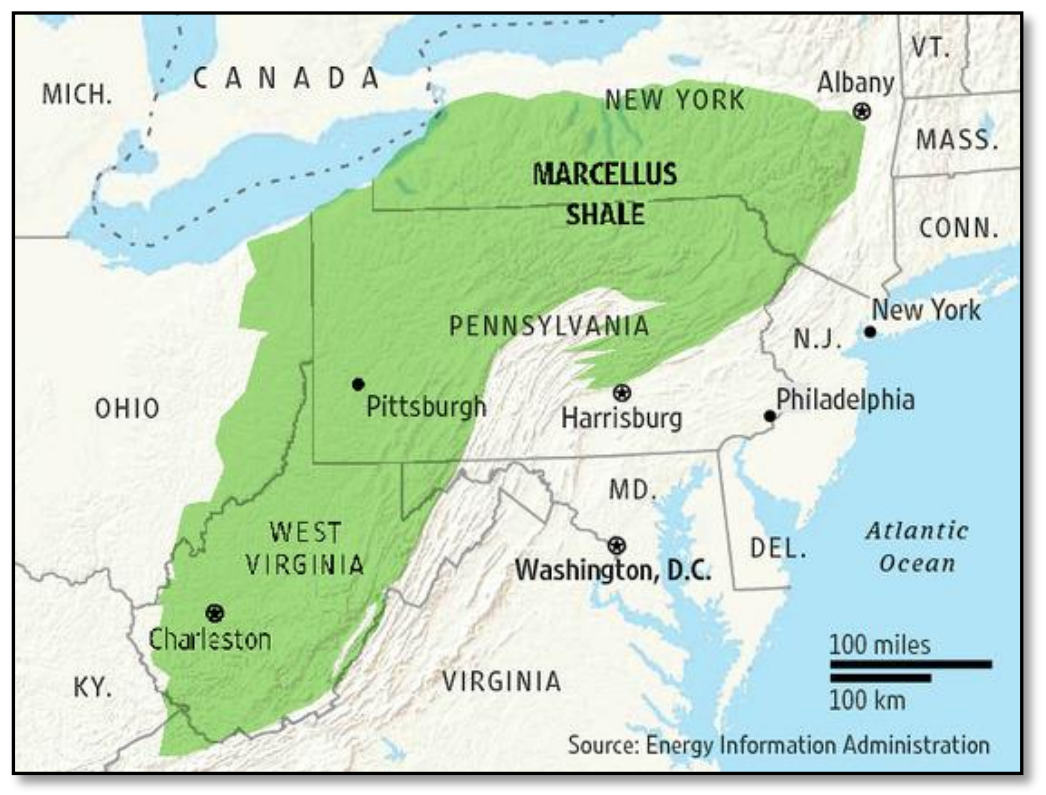

Figure 1: Marcellus Shale Distribution [U.S. EIA] 


\subsection{Problem Statement}

Hydrocarbon production from unconventional shale gas reservoirs has become more common in the past decade. Therefore, prediction of hydrocarbon recovery or estimated ultimate recovery (EUR) for these reservoirs throughout the life of wells is considered the main step in development planning. Decline curve analysis technique is the most popular graphical-mathematical method in the industry used to calculate EUR.

The traditional decline curve technique shows unreasonable results when applied in unconventional reservoirs because of the complexities in petrophysical and mechanical properties of these reservoirs. Consequently, new decline curve models have been developed specifically for tight gas reservoirs. However, both the traditional and new techniques do not consider reservoir characteristics and completion design parameters. But, in reality, reservoir characteristics and completion design parameters have significant impact on future production behavior. Accordingly, study the impacts of these parameters on Estimated Ultimate Recovery (EUR) will provide a better understanding of the reserves estimation.

The main objective of this research is to condition the estimated ultimate recovery of shale wells to reservoir and completion parameters. In addition, it will be determined from this research whether reservoir or completion parameters have the most impact on EUR. The case study includes a large number of wells in a shale asset. The first step is to calculate the EUR for more than 200 shale wells by using traditional and new methods of decline curve analysis.

The results of EUR will be subjected to study and condition with rock properties, well characteristics and completion's design parameters by using artificial neural networks technology. The porosity, total organic carbon, net thickness and water saturation are the main rock properties that are considered in this research. Furthermore, the impact of different well design configurations (for instance, well trajectories, completion and hydraulic fracturing variable) on EUR will be inspected in this study. 


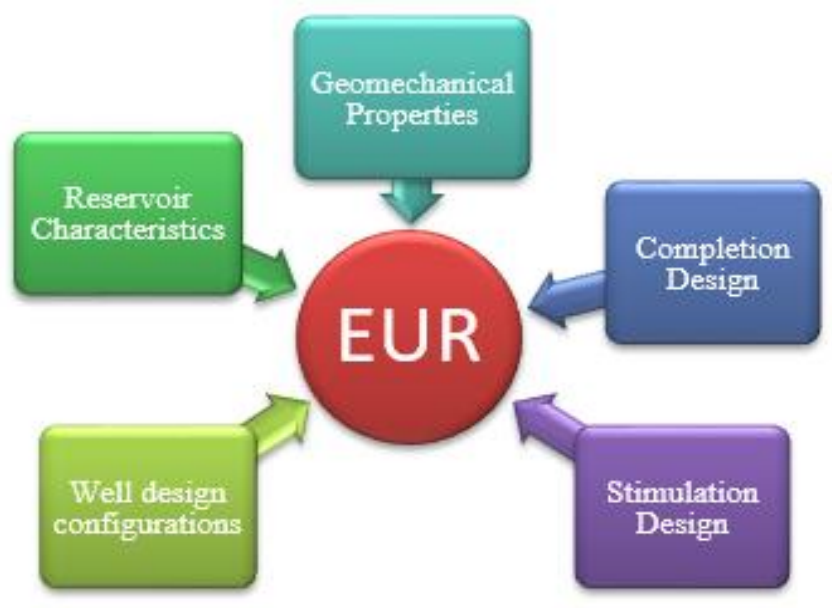

Figure 2: Reservoir and completion parameters

This study differs from other published studies in that the focus was not so much on making comparison in decline curve methods or their results of Estimated Ultimate Recovery (EUR), but rather was on investigating the impacts of different parameters on EUR values.

This study will provide the natural gas professionals insight and clarification regarding the effects of rock properties and well design configurations on estimating the ultimate recovery for gas shale. Also in this study, it will be determined which parameters (reservoir or completion) have more impact on EUR which will give additional understanding to the petroleum industry when planning future well development. 


\section{Chapter 2: Literature Review}

In recent past, unconventional hydrocarbon resources have become very an important energy source in United States and worldwide. Unlike conventional reservoirs, forecasting the future production of unconventional reservoirs is considered a big challenge because of the complexities in petrophysical and mechanical properties of these reservoirs. Therefore, using same conventional tools and techniques that have been used in conventional reservoirs to predict production in unconventional reservoirs is not completely successful idea [Mohaghegh, 2014].

Consequently, various reserve estimation methods have been published in the literature ranging from basic material balance calculations to decline-curve analysis. Reservoir Numerical model which has been used in estimate future production of conventional reservoirs and provide reasonable results shows unreasonable results when applied in unconventional reservoirs [A.J.Clark, 2011]. The reason that some assumptions in this modeling are inconsistent with real field data of these unconventional reservoirs such as pressure initialization and radial transient flow [Duong, 2010].

Simultaneously, empirical techniques which are mathematical relationships of the decline curve analysis have been developed to compute the reserve estimation of the reservoirs. Traditionally, Arps' equation that published by J.J. Arps was commonly used to forecast reserves in conventional reservoirs:

$$
q=q_{i}\left(1+b D_{i} t\right)^{\frac{-1}{b}}
$$

In this approach, Arps considered the equation as empirical, but then realized that the exponent (b) can be influenced by the reservoir flow condition. The degree of the curvature of the semi-log decline is determined by the value of $b$. It becomes a straight line at $b=0$ and increasing the curvature at higher values of $b$. Arps declared that the value of $b$ has to be in range between zero to 1 , but didn't discuss of getting $b$ greater than 1 .

After the Unconventional Reservoirs Revolution, applying Arps' technique for tight gas reservoir showed that the exponent $b$ is greater than 1 [Maley, 1985].As a result the forecasting was overestimation and unreasonable. In that time (around 80s) it was inevitable to apply Arps' method 
to tight gas wells. For example, in1985, Maley was one of the first scientist who applied Arps' equation in tight reservoir. He declared "There is no theoretical basis for limiting the exponent to value less than 1". However, he found in his conclusion that by using exponent greater than 1 , the results showed unreasonable forecasting long well lives. Therefore, he recommended to limit the forecasted production life with some minimum value for the decline rate as attempt to overcome this problem.

Though, in reality, many oil \& gas professionals and engineers avoided using the Arps equation in unconventional reservoir to avoid the overestimation in the investment decisions. Some of them used approach that assumed decline shape composed of a series of straight line segment. This approach is apparently arbitrary [Maley, 1985].

Consequently, in recent studies, several new techniques for the decline curve analysis have been developed to improve the accuracy and reliability of the reserves estimates in unconventional reservoirs and to avoid the overestimation results. For instance, Power Law Exponential (PLE) method which proposed by Ilk et al. [2008] to estimate the ultimate recovery for unconventional reservoir. The interesting side of this model that this model is flexible to model transient, transition, and boundary-dominated flow [Ilk et al., 2008].

Further, other method has been suggested by Valko (2009) which is Stretched Exponential Production Decline (SEPD). The most significant advantage of this model that is the bounded nature of estimated ultimate recovery (EUR) without limits on time or rate [Valko, 2010]. In addition, in 2010, Duong has developed a new empirical model based on long-term liner flow in tight gas reservoir. Duong considers his approach easy and simple to use for predicting the future rate and EUR. Moreover, this model is capable to provide a statistical method to analyze production forecasts of resource and establish a range of results for these forecasts, including probability distribution of the reserves [Duong, 2010].

In this study, these new and the traditional methods have been applied to compute the EUR for more than 200 shale wells. The results of EUR will be subjected to study and condition with reservoir properties and completion's design parameters. The focus is on finding relevant and reasonable trends between these parameters that mentioned above and EUR. In other words, the efforts in this study is to investigate which parameter has significant impacts on future production. 
In addition, it will be determined from this study whether reservoir or completion parameters have the most impact on EUR

Unconventional reservoirs are characterized by very complicated fluid flow because of the complexity in petrophysical and mechanical properties of their rocks. For instance, reservoir characteristics of shale vary quite significantly for same production area. Therefore, using conventional statistical analysis (such as cross-plots and regression) to find relation or patterns in data sets in these reservoirs might be challenging due to their inherent nonlinear behavior [Mohaghegh, 2015]. To overcome this problem, Advance data mining technology has been used to find patterns or relation between the parameters and EUR.

In this study, Advance data mining technology called Fuzzy Pattern Recognition since it is based on Fuzzy theory [Mohaghegh, 2000] has been employed to discover any hidden patterns or reasonable trend between parameters such as porosity, total organic carbon, net thickness, water saturation, well trajectories and hydraulic fracturing design with EUR.

Pattern Recognition is a branch of artificial intelligence used for classification or description of observations. The objective of the Pattern Recognition is to classify data based on the statistical information that extracted from the pattern. 


\section{Chapter 3: Background}

\subsection{Introduction}

In any Reservoir Management or Development, the main question will be risen that how much oil and/or gas this reservoir be able to produce? How many years this well will be able to produce? Defiantly, the forecasting and simulation studies will be employed to answer these questions. In general, there are three techniques that used for forecasting such as Volumetric, Material balance and decline curve analysis. As mentioned earlier, using same conventional tools and techniques that have been used in conventional reservoirs to forecast production in unconventional reservoirs is not successful idea [Mohaghegh, 2014].

In addition, Duong, 2010, mentioned in that some assumption that used in the modeling conventional reservoirs are inconsistent with real field data of unconventional reservoirs such as pressure initialization and radial transient flow. Moreover, most the results of estimation future production obtained by using reservoir numerical modeling on unconventional reservoirs are unreasonable. Therefore, estimated ultimate recovery by using decline curve analysis is common method in the oil industries because it is simple to use, requires less data and provides reasonable results.

\subsection{Decline Curve Analysis}

Decline curve analysis is an empirical method used in reservoir engineering to extrapolate tendencies in the production data from oil and gas wells and to make a forecast of coming production rates in addition to determine the Estimated Ultimate Recovery (EUR). The decline curve analysis was performed by plotting rate versus time or rate versus cumulative production. The most traditional methods of decline curve equations are those first notarized by J.J. Arps (1945). These methods are only valid during Boundary-Dominated Flow (BDF) that typically occurs in conventional reservoir when transient flow period ends after a few days. During transient flow, these methods do not apply (see figure 3).

In contrast, unconventional tight reservoirs, that have low permeability and require extensive fracture networks to produce, can have transient periods between several months to several years 
till reach the BDF. As a result, Arps' equations do not work perfectly in unconventional reservoirs and could result overestimate EUR (see figure 4).

Consequently, in recent studies, many researchers have tried to solve this problem by derivative equations that involve both transient and boundary-dominated flow. In other word, these equations are particularly applicable to tight unconventional reservoirs because, as mentioned, the transient flow is often the dominant flow regime, and the boundary dominated flow may not be reached until after many years.

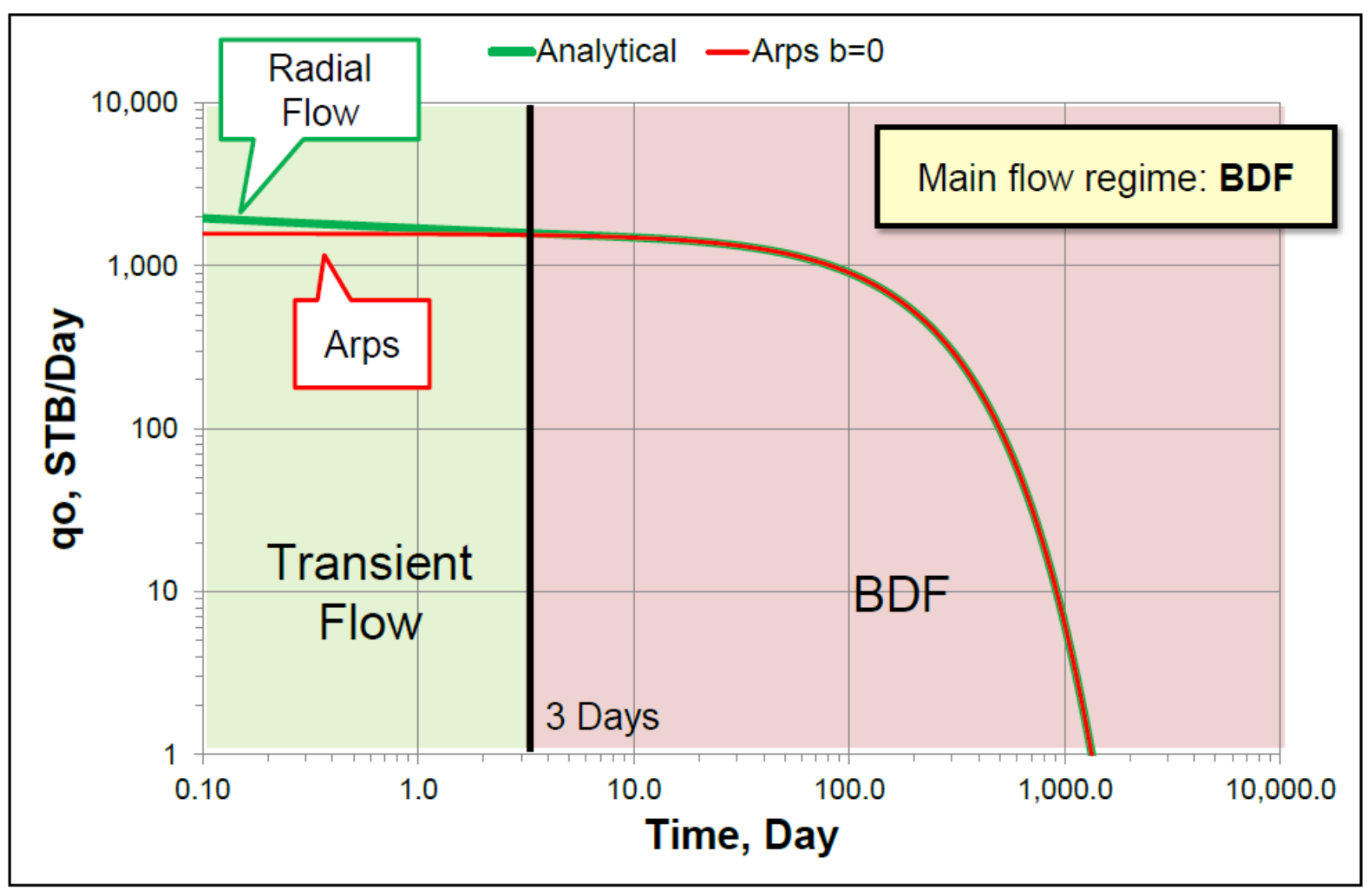

Figure 3: Applying Arps' equations in conventional reservoir [Courtesy of Reference 1] 


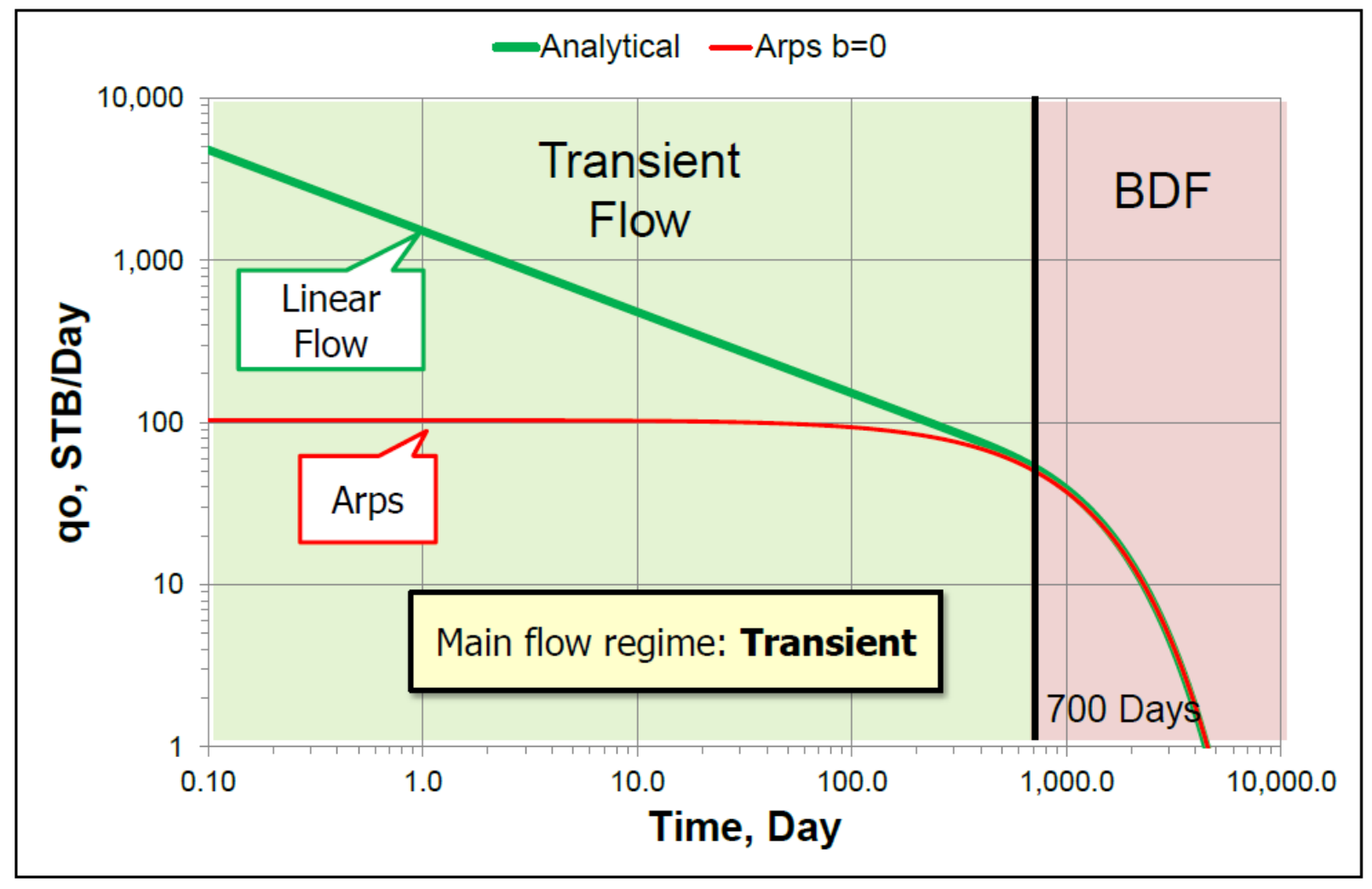

Figure 4: Applying Arps' equations in unconventional reservoir [Courtesy of Reference 1]

\subsubsection{Traditional Arps Decline curve Analysis}

Arps's equations, published by J.J. Arps in 1945, are the most common traditional techniques used to calculate Estimated Ultimate Recovery (EUR) for conventional reservoirs because they are simple to use and require less data. Arps classified three types of production rate decline during BDF (Exponential, Hyperbolic and Harmonic) depending on the way that the nominal decline rate (D) changes with rate in a semi-log plot (log q vs. t). In other word, the changing in the decline degree base on the value of the decline exponent constant $b$. Arps derived the equations for each type by using the concept of loss-ratio and its derivative.

Loss ratio:

$$
\frac{1}{D}=-\frac{q}{d q / d t}
$$


The loss ratio derivative:

$$
b=\frac{d}{d t}\left[-\frac{q}{d q / d t}\right]
$$

The flow rate equation:

$$
q=q_{i}\left(1+\mathrm{b} D_{i} \mathrm{t}\right)^{-1 / b}
$$

In above equations, "q" refers to flow rate, "qi " refers to initial flow rate, "D" is how steep the decline is, "b" is the leveled out portion of the curve, and " $\mathrm{t}$ " is the production time such as day or month.

Arps declared that the value of $\mathrm{b}$ should lie between 0 and 1.0 and did not discuss of possibility $\mathrm{b}$ greater than 1. Mathematically, the value of $b$ determines the degree of curvature of the decline. Start from straight line (exponential decline) when $b=0$ to increasing curvature at higher value of b. (see table 1)

\begin{tabular}{|l|l|l|l|}
\hline & \multicolumn{1}{|c|}{\begin{tabular}{c} 
Exponential \\
\cline { 2 - 4 }
\end{tabular}} & \multicolumn{1}{c|}{ Hyperbolic } & \multicolumn{1}{c|}{ Harmonic } \\
\cline { 2 - 4 } & $\mathrm{D}=$ constant & $\mathrm{D}=\mathbf{b} \leq \mathbf{1}$ & $\mathrm{D}=$ changing \\
\hline Flow rate equations & $q=q_{0} e^{-t D_{i}}$ & $q=q_{0}\left(1+b D_{i} t\right)^{\frac{-1}{b}}$ & $q=q_{0}\left(1+b D_{i} t\right)^{\frac{-1}{b}}$ \\
\hline Cumulative equations & $Q=\frac{\left(q_{0}-q\right)}{D_{i}}$ & $Q=\frac{q_{0}{ }^{b}}{D_{i}(1-b)}\left(q_{0}^{1-b}-q^{1-b}\right)$ & $Q=\frac{q_{0}}{D_{i}}\left(\log q_{0}-\log q\right)$ \\
\hline
\end{tabular}

Table 1: Arps' equation based on b values

However, for unconventional reservoirs, it is often observed that values of $b$ is greater than 1.0. In 1985, Maley applied Arps' equations for tight gas (unconventional reservoir) and mentioned in his conclusions that the exponent $b$ in Arps' equations often does exceed 1.0. Moreover, many studies show that applying Arps' equations in unconventional reservoirs give $b$ value greater than 1.00 such as Ilk, in 2008, stated that often observe b value greater than 1.0 when applied Arps' 
equation for tight gas. In addition, in this study Arps' equations have been applied on more than 200 shale gas wells and show b- exponent value much greater than 1 .

As mentioned before that $b$ value determines the curvature for the decline. Therefore, when $b$ value is greater than 1.0, the decline curve decreases and, in consequence, the results of the forecasting show overestimation. To overcome these problems, recently, new models of decline curve have been developed to estimate ultimate reserve of tight gas shale or in general unconventional reservoirs.

\subsubsection{The New Decline Curve Models}

As discussed before, the Arps' equations are not valid to apply in unconventional reservoirs for different reasons (the long period of the transit flow in unconventional reservoir and the range of b-exponent should stretch between 0 and 1 for Arps equations). However, the studies observed that b-exponent value is greater than 1 for unconventional reservoirs. Consequently, many researches tried to develop new equations that have $b$-exponent changes with time and are able to handle $b$ greater than 1.0. Two of those new methods will be covered in this section, Power Law Exponential Decline by Ilk, 2008 and Stretched Exponential Production Decline by Valko, 2009.

\subsubsection{Power Law Exponential Decline Models (PLE)}

Ilk et al. (2008) established the Power Law Exponential (PLE) decline model, which is an improvement of Arps' exponential decline, to get better fit and forecast of EUR specifically for low permeability (unconventional reservoirs).

PLE loss-ratio,

PLE derivative of loss-ratio,

$$
\mathrm{D}=\mathrm{D}_{\infty}+\mathrm{n} \mathrm{D}_{\mathrm{i}} \mathrm{t}^{\mathrm{n}-1}
$$

$$
\mathrm{b}=\frac{\mathrm{d}}{\mathrm{dt}}\left(\frac{1}{\mathrm{D}}\right)=\frac{-\mathrm{n}(\mathrm{n}-1) \mathrm{D}_{\mathrm{i}} \mathrm{t}^{\mathrm{n}-1}}{\mathrm{D}^{2}}
$$

PLE rate-time relation,

Where:

$$
q=q_{i} \exp \left(-D_{\infty} t-\frac{D_{1}}{n} t^{n}\right)
$$

$\mathrm{q}_{\mathrm{i}}=$ rate intercept at $\mathrm{t}=0$

$\mathrm{D}_{\infty}=$ decline constant at infinite time

$\mathrm{D}=$ decline constant

$\mathrm{n}=$ time exponent 


\subsubsection{Stretched Exponential Production Decline Model (SEPD)}

Valk (2009) developed a new equation which has a different technique to handle the exponent value. This equation is called Stretched Exponential Production Decline Model (SEPD). When nexponent (equation 8) is set equal to one in SEPD, the equation results in an exponential decline and by decreasing $n$-exponent value the depletion gets slower which is completely opposite as what is found when using the Arps decline method (see figure 5). All these characteristics give the SEPD method a more rational estimate of EUR compared with other methods.

SEPD rate-time relation,

$$
q=q_{i} \exp \left[-\left(\frac{t}{\tau}\right)^{n}\right]
$$

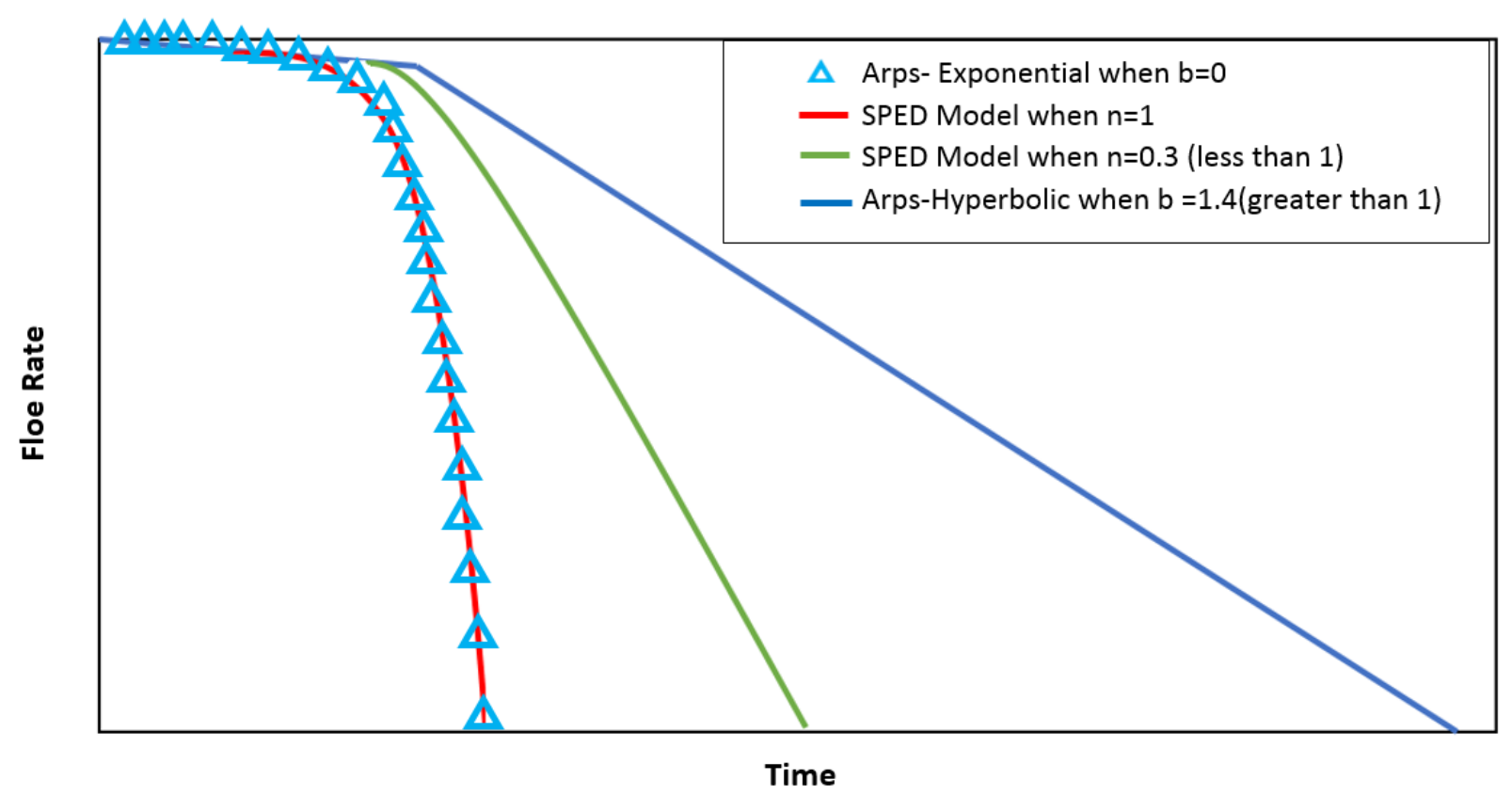

Figure 5: Stretched Exponential Decline Model Behavior [Courtesy of Reference 2] 


\subsection{Artificial Neural Networks}

The failure in solving complex problems by using conventional digital computers have brought a new technique called Artificial Neural Networks. An Artificial neural networks (ANNs) are defined as model designed to simulate the way that the human brain processes information. The concept of ANNs comes from how the human brain is capable of solving complex problems without using a mathematical model but rather through using cognitive observation and learning from experiences. Thus, The Artificial Neural Networks do not use algorithmic processes that used in conventional algorithmic computers, instead, they react to things learned through experience. Accordingly, it is important to provide the ANNs with sufficient examples to learn the neural networks.

The Artificial Neural Networks are designed in a similar way to how neurons in human brain process the information. In the human brain, the neuron gathers signals from other neurons through a network of fine structure called dendrites. Then, the neuron sends waves of electrical pulses through long and thin strands within the brain called axon. This axon is split into branches and at the end of each branch are the synapse. This synapse translates the electrical pulse from the axon and returns another electrical pulse that will speed up or slow activity in the axon and thus, the connected neurons. It is at this time, if the neurons receive enough input, they will send electrical activity back through the axon. Learning actually happens by changing the productiveness of the synapses in order to change the influence of one neuron on another. The result of that an intelligent brain capable of learning, prediction and recognition. Figure (6) shows schematic of a biological neuron.

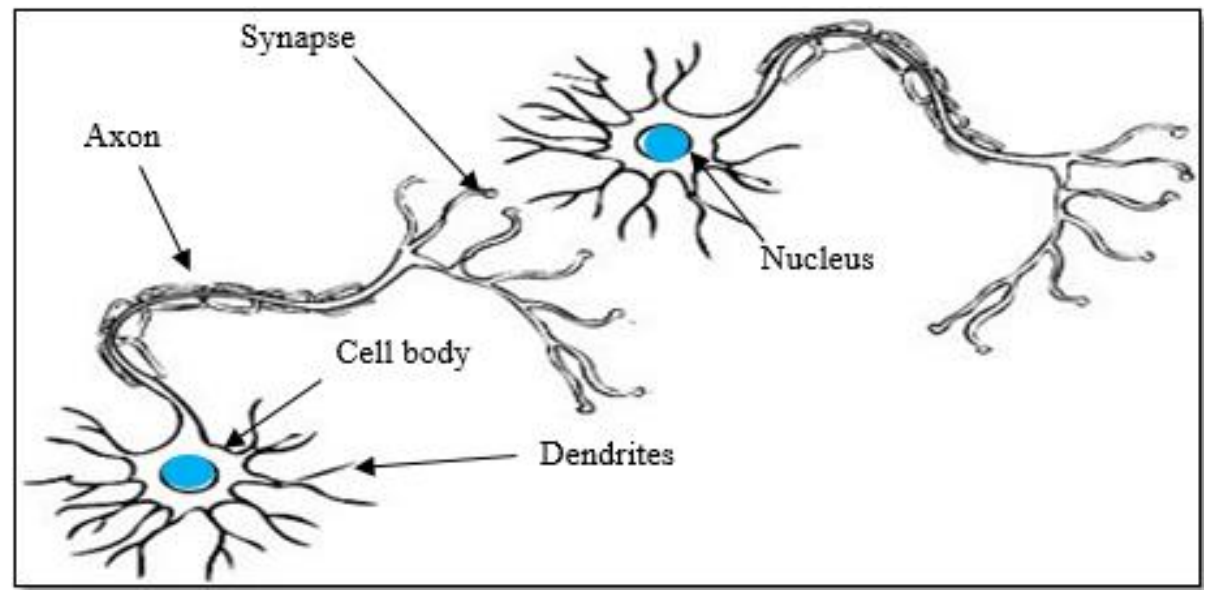

Figure 6: Neuron Cell (https://sites.google.com/site/drhuntersanatomyandphysiology/neurons) 
In other hand, the Artificial Neural Networks basically have neurons and each single neuron consist of inputs (like synapses). The inputs are multiplied by weights, and then summed by a this activation function which determines the activation of the neuron. After that, they passed through a transfer function (which called transfer function) to produce the output for that single neuron. It has to mention here that the computation of the neuron are different because they are impacted by the weight. Therefore, to achieve the desired output for specific input, the weight should be adjusted. Figure (7) shows mathematical structure of neuron.

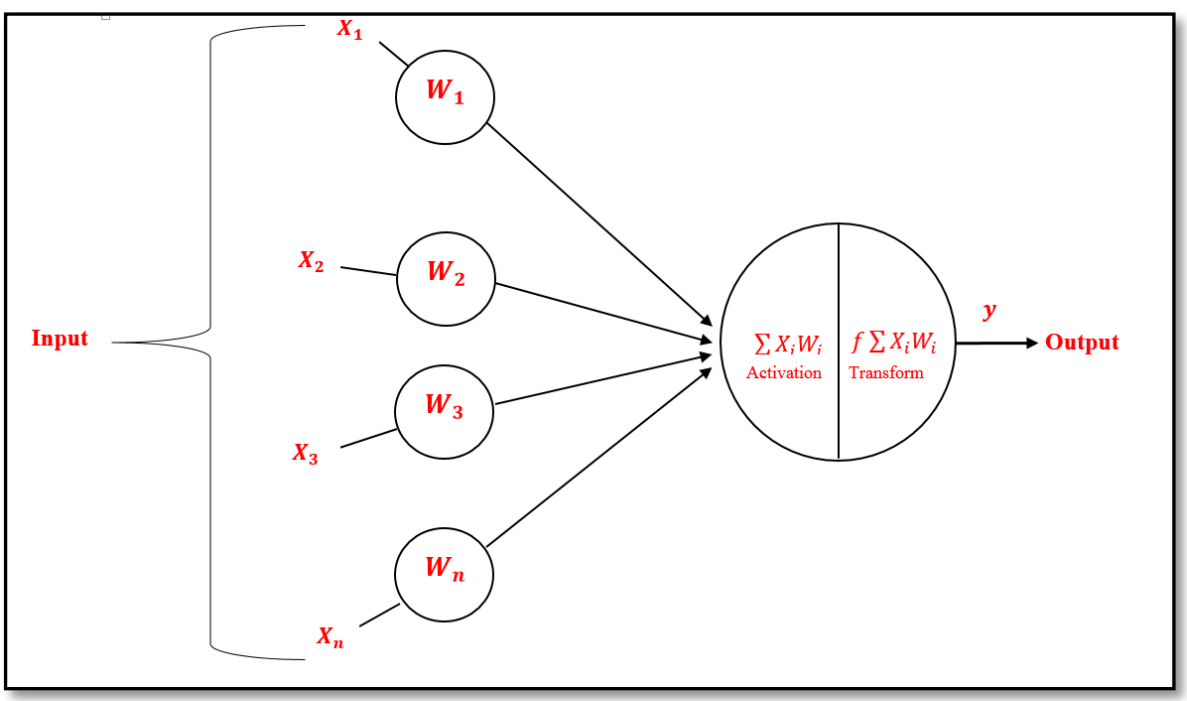

Figure 7: Mathematical structure for neuron of Artificial Neural Networks

The neurons are connected to each other by different ways. These connection ways have significant effect on the operation of artificial neural network. The Feedback and Feedforward are the most popular connection ways that used in the ANNs. In the Feedback, the outputs of one layer (neurons are arranged in one layer) send back to the input of a previous layer, or to same layer (see figure 8).

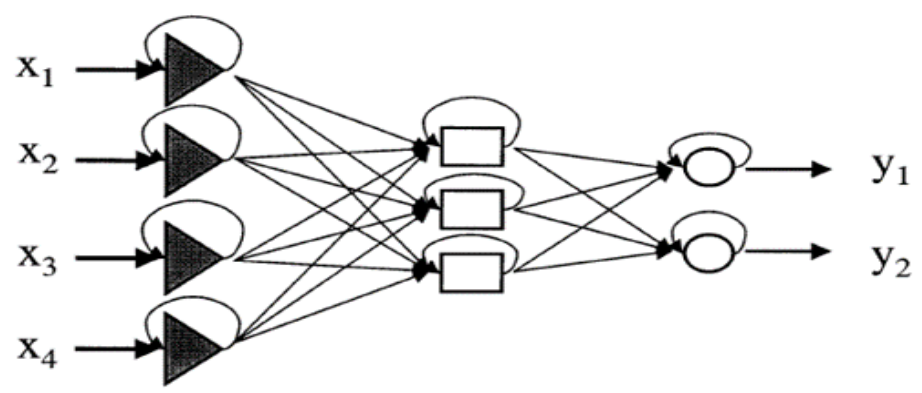

Figure 8: Feedback network (www.elsevier.com:locate:jpba) 
In other side, Feedforward are not designed to send the output back to the input neurons as can be seen in Feedback process. Therefore, the Feedforward cannot retain its earlier output values. Figure (9) shows diagram of Feedforward.

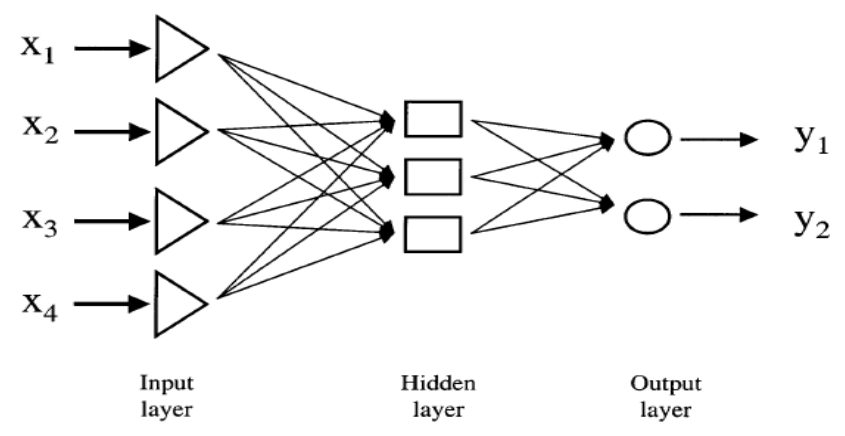

Figure 9: Feedforward network (www.elsevier.com:locate:jpba)

As human's brain the neurons are trained and learned by experiences, the ANNs have to be learned and trained by sufficient training. The neural network, with sufficient number of neuros and sufficient training, can model any nonlinear functions [Cybenko, 1989: Funahashi, 1989].

One of the most often technique that used to learn the network is Backpropagation. Data from inputs are fed forward the neural network to optimize the weight through the neurons. Then, the backward propagation is used to optimize the weight by minimizing the error during the training or learning process. The progress of Backpropagation starts when the ANNs read the inputs and outputs in the training process then alter the weight to reduce the error of the different between the predicted or calculated value by ANNs and the output that inserted to ANNs. This error is minimized as much as possible by training cycles till reach very small different between the predicted and the actual (output )values. Then, the ANNs are called trained enough for using. Figure (10) shows diagram of Backpropagation process.

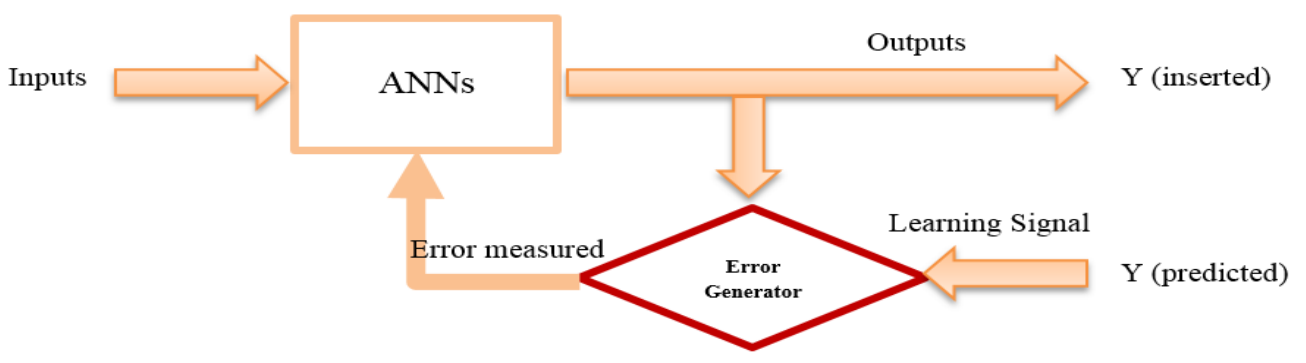

Figure 10: Backpropagation Training Diagram 
The important applications of neural networks are pattern recognition and data classification. The pattern recognition technique can be used to extract pattern or hidden trend from complex data when the computer techniques are unable to find it. For example, in oil\& gas industries, studies have been shown that unconventional reservoirs have very complex properties, unlike the conventional reservoirs, and the correlations that used in conventional reservoirs cannot be applied in unconventional reservoirs.

Therefore, this complex system, nonlinear system, needs new technique that is capable of processing extensive amounts of complex dataset. In other words, new technique has the ability to find trends and useful information from large datasets. As a result, using the Artificial Neural Network as new technique (compared with conventional digital computers) in these reservoirs shows successful approach because the Artificial Neural Network has proven to have potential in solving problems that require pattern recognition.

In this study, Fuzzy Trend Analysis (FTR) and Well Quality Analysis (WQA) which are branches of Artificial Neural Network concerned with classification and observations are employed to discover any hidden but useful patterns among non-linear and interdependent parameters involve in the shale gas datasets. 


\section{Chapter 4: Methodology}

Before starting in the methodology of this study, it is necessary to remark some important points. First of all, as mentioned earlier, the main focus of this thesis is to condition the Estimated Ultimate Recovery (EUR) with reservoir and completion parameters. In other words, the objective of this study is to find trend or relation between these parameters and EUR.

This study differs from other published studies in that the focus was not so much on making comparison in decline curve methods or their results of Estimated Ultimate Recovery (EUR), but rather was on investigating the impacts of different parameters on EUR values.

Another aspect of this study which differs from other published studies is that the study is applied on real field data and, therefore, based on fact. In this case, the process is dealing with highly variable flow rates (noisy data), different operation condition and other factors that have effects on production behavior.

The following procedure that explain in the below flowchart (figure 11) is designed to give consistent, reasonable results:

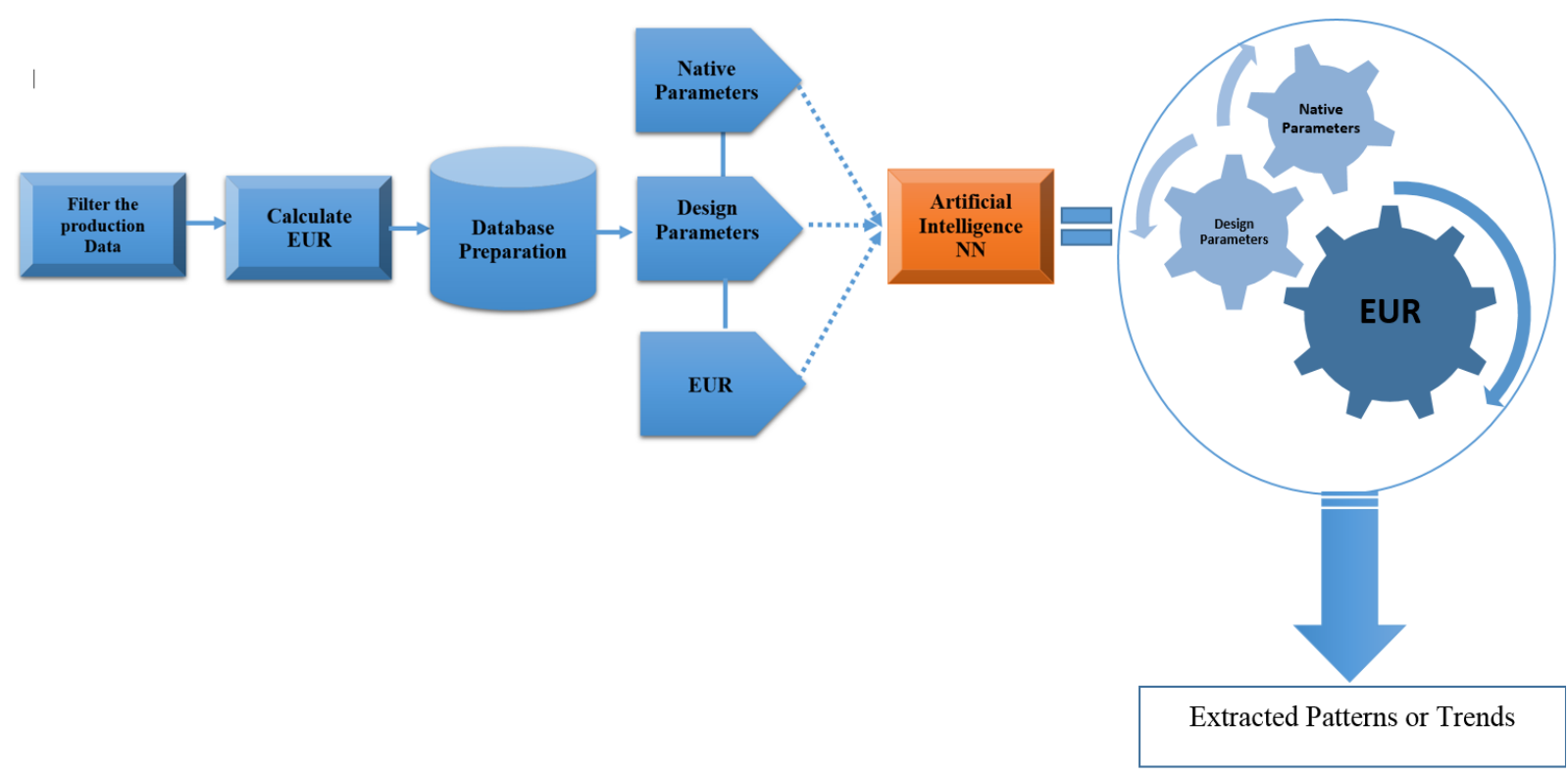

Figure 11: Flowchart shows the steps of the methodology 


\subsection{Filtering the Production Data}

The EUR quality of both the traditional and the new models is limited by the quality of the analyzed data. In practice, the single well production is subjected to different factors that effect on the flow rate of the well such as changing production strategies or changing operation conditions. Since, this study is applied on real field data, it is hard to find reasonable trend of flow rate decline profile. Therefore, the big challenge here is to make the noisy data reasonable data.

For all these points that mentioned above, filtering the Production Data is considered to be the rock stone of getting accurate EUR in any study. The following steps are applied on the every single well before calculate the EUR.

$\square$ Highlight the reasonable trend

$\square$ Ignore any points from different production strategies or changing operation conditions

$\square$ Ignore points that considered producing below the production capacity of the well in just portion of the month. 


\subsection{Calculating the Estimated Ultimate Recovery (EUR)}

Estimated ultimate recovery have been calculated for more than 200 shale gas wells by using decline curve analysis to predict production for 10, 30 and 50 years. With these large number of wells, the study becomes more reasonable and logical. Both the traditional and the new models of the decline curve analysis that mentioned earlier have been used in this study to calculate EUR. In this way, this study will be based on both traditional and modern techniques of calculating EUR. The Optimism and Pessimism in Decision Making is Based on the way that EUR calculated.

The two traditional models:

- Hyperbolic Model (HB)

- $\quad$ Tail End Exponential Model (TED)

$$
\begin{aligned}
& q=q_{i}\left[\left(1+b D_{i} t\right)^{(-1 / b)}\right] \\
& q=q_{i} \operatorname{Exp}\left[-t D_{i}\right]
\end{aligned}
$$

The two new models:

- $\quad$ Power Law Exponential Model (PLE)

- Stretched Exponential Production Decline Model (SEPD)

$$
\begin{aligned}
& q=q_{i} \operatorname{Exp}\left(-D_{\infty} t-\frac{D_{1}}{n} t^{n}\right) \\
& q=q_{i} \operatorname{Exp}\left[-\left(\frac{t}{\tau}\right)^{n}\right]
\end{aligned}
$$

Non-linear regression technique, that introduced by Towler 2002, has been used on an EXCELLSOLVER for determining decline curve parameters. A spreadsheet was set up containing cells for each parameter of every model of the Decline Curve Analysis (DCA). For every model, several columns in Excel Sheet have been created which are actual production data $q$, appropriate production rate equation $q^{-}$, the errors squared $\left(q-q^{-}\right)^{2}$ and total errors squared $\left(q-q_{\text {Average }}\right)^{2}$.

Moreover, a cells for the sum of errors squared $S_{S E}$ and total errors squared $S_{S T}$ were created. Finally, a cell for the regression coefficient was generated by using the following formula:

$$
R^{2}=1-\frac{S_{S E}}{S_{S T}}
$$


By using Microsoft Excel's built-in Solver function and setting the objective cell containing $R^{2}$ equal to one while changing the appropriate variable cells for each DCA model, the best fit to simulated production data could be obtained.

Once the above columns and cells in Excel are created, the Non-linear regression technique can be applied by using Microsoft Excel's built-in Solver function. This was by setting the objective cell containing $R^{2}$ equal to one. Then, the calculations are started by changing the parameters of each DCA till obtain the appropriate variable cells for each DCA model and as a consequence the best fit to actual production data is found. The following graph show an example of applying the four models and their differences in estimated ultimate recovery for 10, 30 and 50 years.
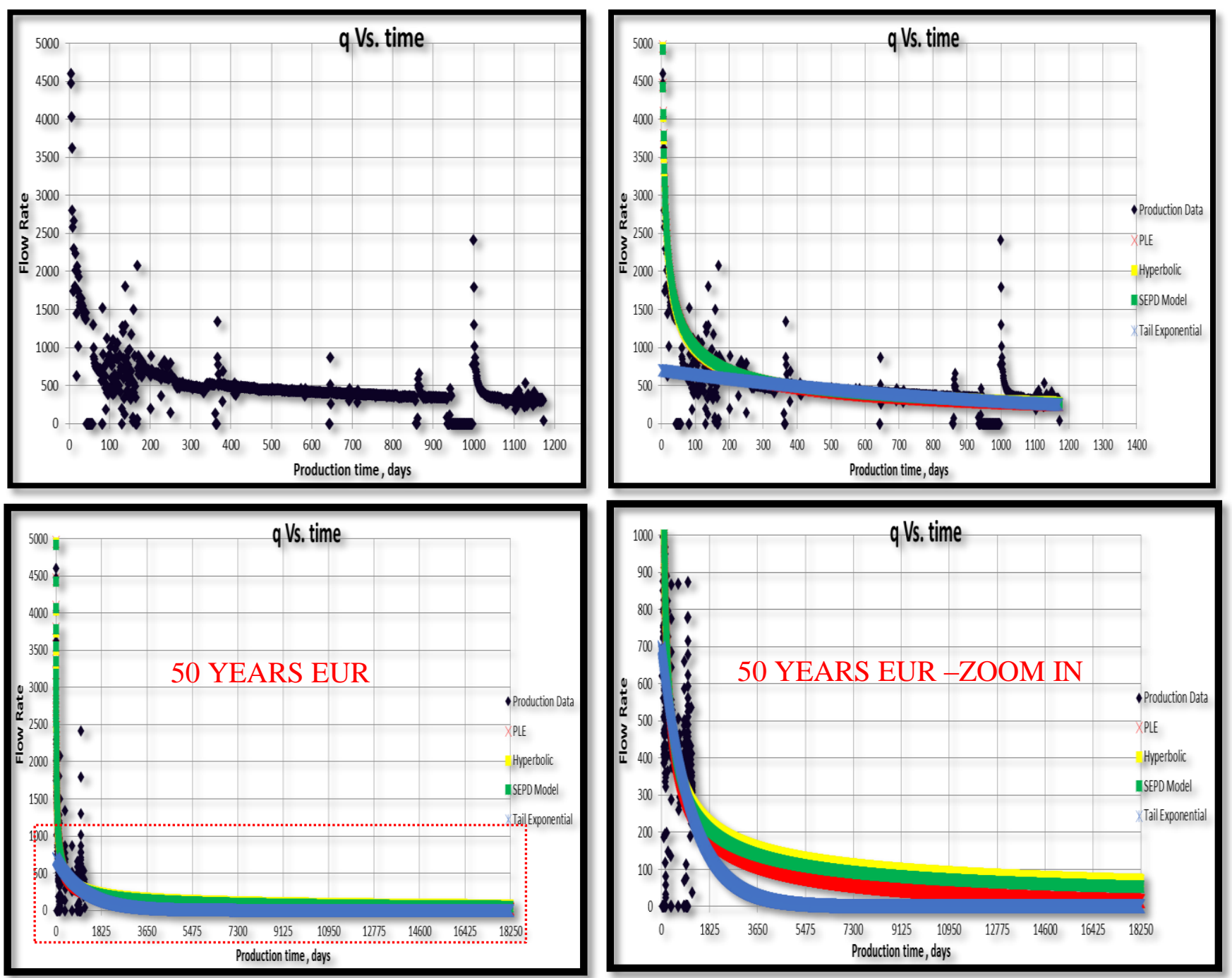

Figure 12: Applying the Four Models on Field Data- Well \#10030-1 
The above figure (12) shows the results of applying the four models on Well \#10030-1. It can be seen that Hyperbolic models give highest EUR. In other hand, Tail End Exponential model provides the lowest value of EUR. Meanwhile, the new models PLE and SEPD predict reasonable results.

\subsection{Preparing the Database}

As mentioned earlier, this study is applied on large number of shale wells. Also, the objective is to condition the Estimating Ultimate Recovery (EUR) of Shale Wells to Reservoir and Completion Parameters. These parameters which considered in this study are taken from large dataset for more than 200 shale wells such as reservoir characteristics, geomechanical properties, completion and stimulation data.

This study is applied on more than 200 shale wells in Marcellus shale located in Pennsylvania. These wells are described by multiple pads, different landing targets, diverse reservoir properties and different completion and stimulation information. The diversity of the wells and reservoir properties give this study comprehensive insight and extensive knowledge for the impact of different parameters on EUR. In other words, this diversity will cover most completion designs and reservoirs properties that are existing in the oil fields.

The dataset that are subjected to study in this research are classified based on the ability to modify them to Design parameters which can be changed and Native parameters which can not be changed. The design parameters include the completion and stimulation design such as the shot density, perforated/stimulated lateral length, number of stages and etc which represent the completion data. The stimulation design are the amount of injected clean water, rate of injection, injection pressure, amount of injected slurry and etc. Because the data was provided on a per well basis, a cumulative total of volumes of fluid and proppant for multiple hydraulic fracture stages were recorded while the pressures for each hydraulic fracture stage were averaged.

In other side, the Native parameters are well information and the reservoir characteristics which are matrix porosity, matrix permeability, pay thickness, net to gross (NTG), initial water saturation and total organic content (TOC). In addition, the geomechanical properties are provided such as 
shear modulus, minimum horizontal stress, young's modulus, and poisson's ratio. All these dataset that mentioned above are supplied for each single well. Figure (13) shows these parameters that are considered in this study.

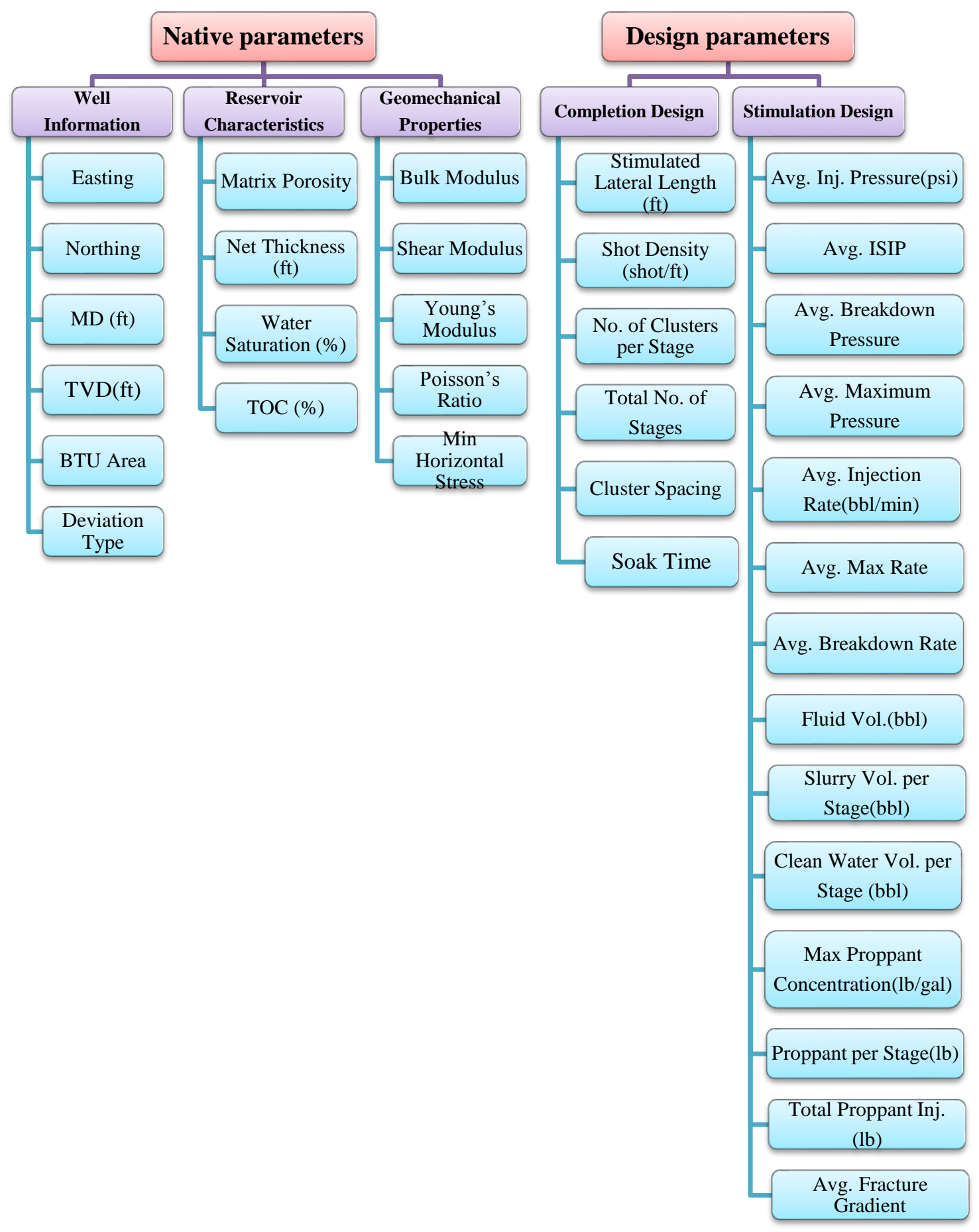

Figure 13: Native and Design Parameters 


\subsection{Artificial Neural Network}

Artificial Neural Network (ANN) was used in this study to find the relation or trend between EUR and parameters. Pattern recognition which is a branch of the artificial intelligent neural network is applied in this research to discover any hidden but potentially useful pattern in the dataset that are subjected to investigate in this study. Improve software has been employed to achieve this goal. IMproveTM software, which was developed for artificial neural network task, was employed to discover the hidden pattern and achieve the goal of this study.

The processes stared by determining the input and output for the model. In this study, 34 parameters are the input and only EUR is set the output. EUR have been calculated by using the four models that are mentioned earlier in the Decline curve section. However, studying the impacts of all these parameters on EUR give very complex model. To avoid the complexities in the model, Key Performance Indicators" analysis was run to determine the influence of each parameter (input). Then, the parameters that have highest impact on EUR are selected.

\subsubsection{Key Performance Indicators}

Key Performance Indicator, or KPI, analysis uses to approximate what level of influence a given input will have on the output (EUR). A value between 0 and 100 is assigned to each input as its "Degree of Influence". In this study, the top parameters that show the highest influence on EUR were subjected to investigate and condition with EUR. Figure (14) shows the KPI result model built. 


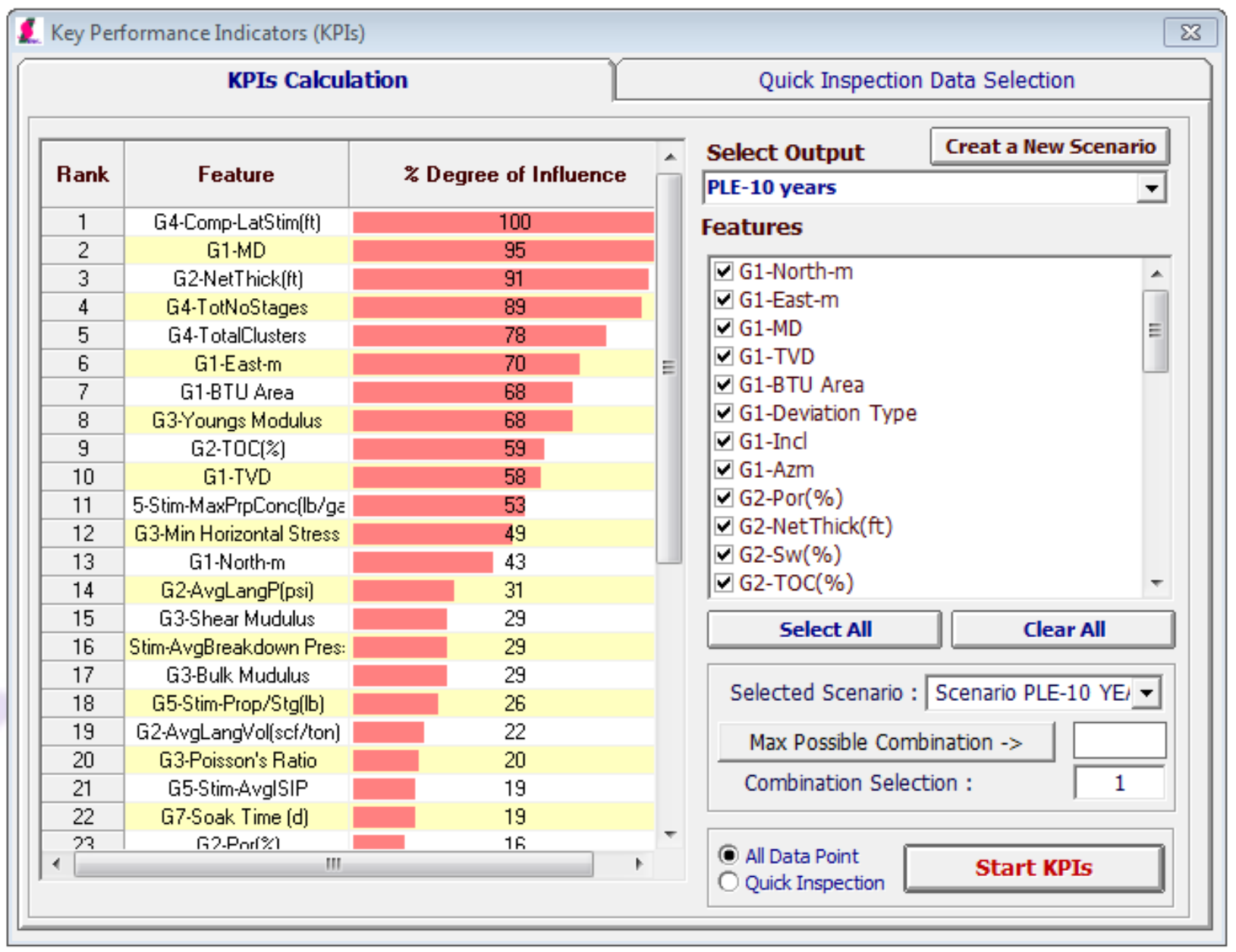

Figure 14: Key Performance Indicators

KPI analysis is an excellent and easy technique to determine what inputs should be selected for the neural network (the inputs have highest impact on output EUR). However, the results of the KPI analysis should not be the final decision when determining which parameters have highest effects on EUR. Some knowledges about reservoir engineering should be used as well. Parameters such as porosity, permeability, water saturation, pay thickness, and so on all play a big role in production and EUR. KPI analysis may not recognize the impact of all these parameters.

IMprove $^{\mathrm{TM}}$ software has another technique used to find hidden pattern and select the input called Fuzzy Pattern Recognition (FPR). All the KPI, FPA and reservoir engineering knowledges are used to determine the most effective parameters as an input for neural network. 


\subsubsection{Data Partitioning and Neural Network Training}

Training the model is very important step in Artificial Neural Networks. In this study, the neural network is prepared for training by selecting the inputs that the KPI analysis showed as having a high influence. In addition, based on knowledges, other inputs that are expected to have a significant influence on EUR are selected.

In Artificial Neural Networks, after selected inputs, the dataset has to be divided into three sets which are Training, Calibration and Verification. This step is called partitions. In this study, 205 wells represent the dataset of the model. Training, Calibration and Verification Sets are created by using an Intelligent Data Partitioning. The data is split into a 10\% Calibration, 10\% Verification and $80 \%$ Training data sets. In this case, the model will be prevented from over training. Once the partitions have been generated, training the neural network can start.

The BackPropagation Neural Network technique was utilized for training mechanism in this research which provided by IMPROVE ${ }^{\mathrm{TM}}$. This technique is one of most popular learning algorithm used in the ANN. In feed-forward backpropagation, there are three parameters are responsible for algorithm convergence which are momentum, learning rate, and weight decay. Momentum and Learning Rate of the neural network have been selected when the BackPropagation Neural Network was designed in this model. IMPROVE ${ }^{\mathrm{TM}}$ defaults these values and the default values are used. Using the default values are recommended because these default values are based on many years of experience in working with different cases of neural network models. As mentioned earlier, usually the neural networks have three layers which are input, output, and hidden layer.

The number of neurons in the hidden layer can also be changed however the default value has been used which was determined by the total number of the inputs parameters. Figure (15) shows the Design of the BackPropagation Neural Network. 


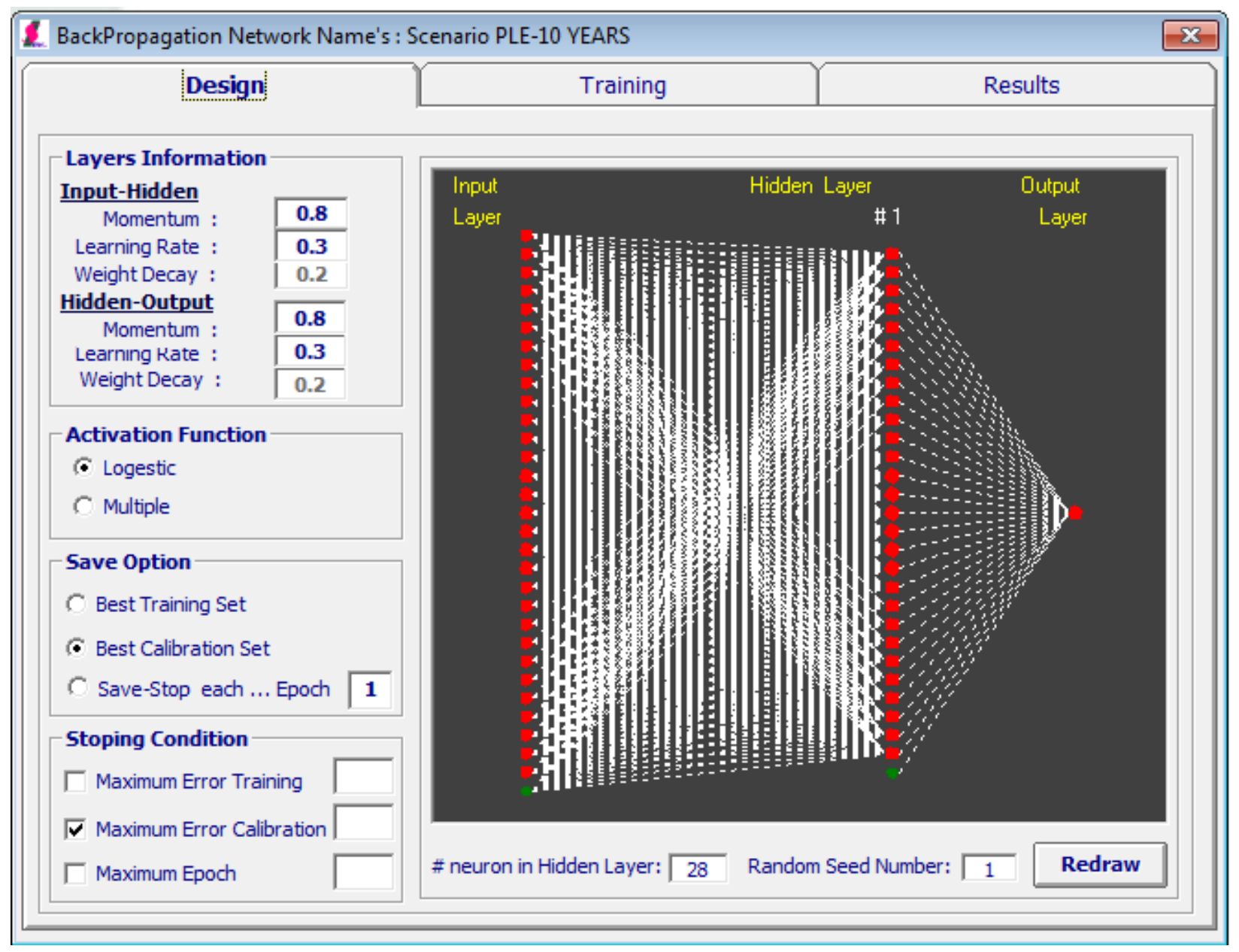

Figure 15: Design for the Back-Propagation Neural Network

Once the design is set, the network model is ready to begin training. As mentioned before, $80 \%$ of data was used for training the neural network and $10 \%$ for calibration and $10 \%$ validation. Iterations or 'epochs' of the NN were established until the error calibration or error training no longer reduces. Figure (16) shows the training process of the Neural Network. 


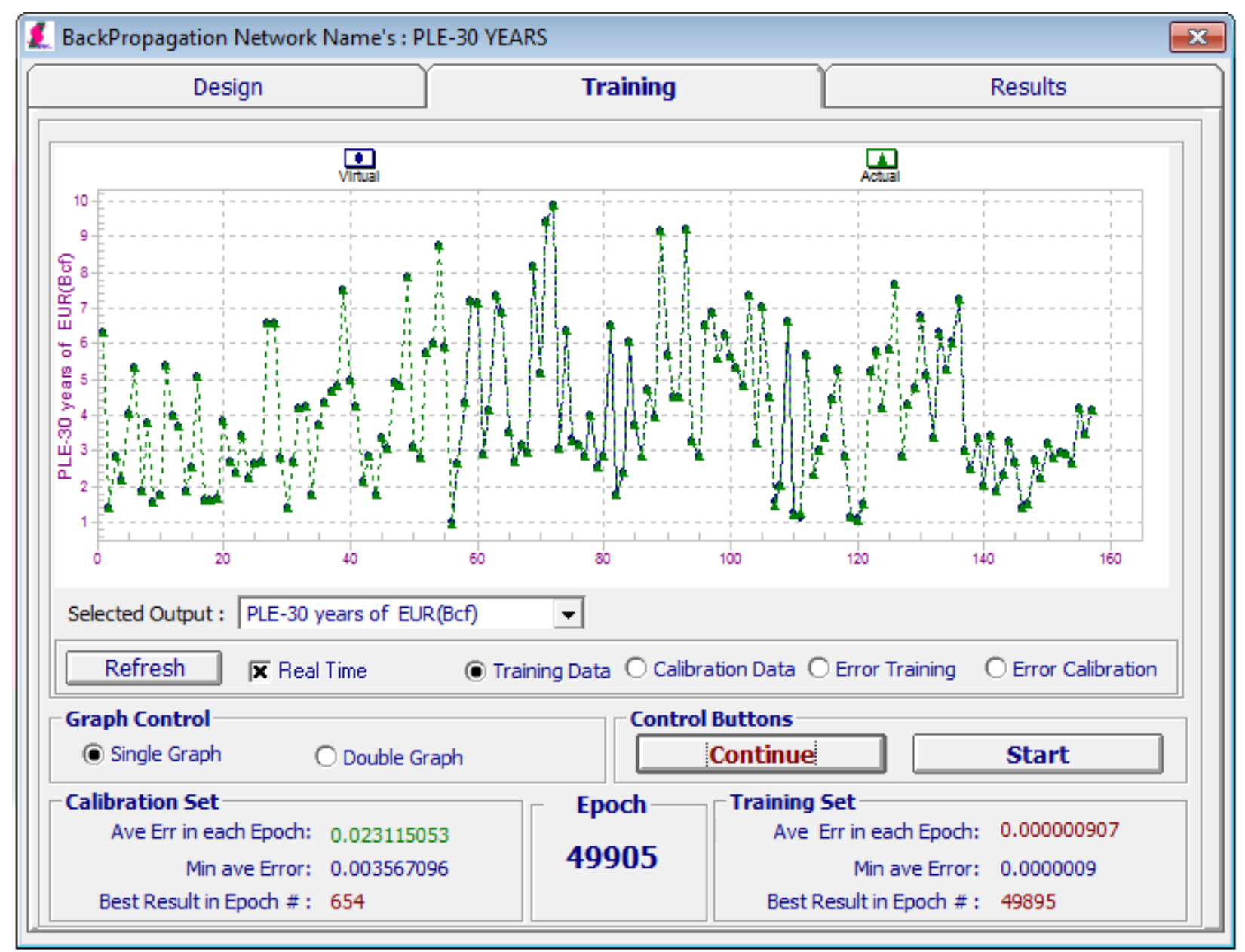

Figure 16: The training process of the Neural Network (PLE MODEL)

The best result of training the Neural Network should show a good match with actual output values. In other words, the acceptable model should have R-squared value above 70\%. By reaching a good match with the actual data or getting high R-squared, the ability of predicting and discovering the hidden trends in the dataset are increased. For this reason, it was important to train the model till get high R-squared.

Figures (17) and (18) illustrate the cross and the scatter plots respectively of a trained neural network for 10-year-EUR with R-squared of 0.86 for all cases. Figures (19) and (20) show the cross and the scatter plots respectively. From these figure (19), (20), it can be seen the quality of training process which has $0.90 \mathrm{R}$-squared. 


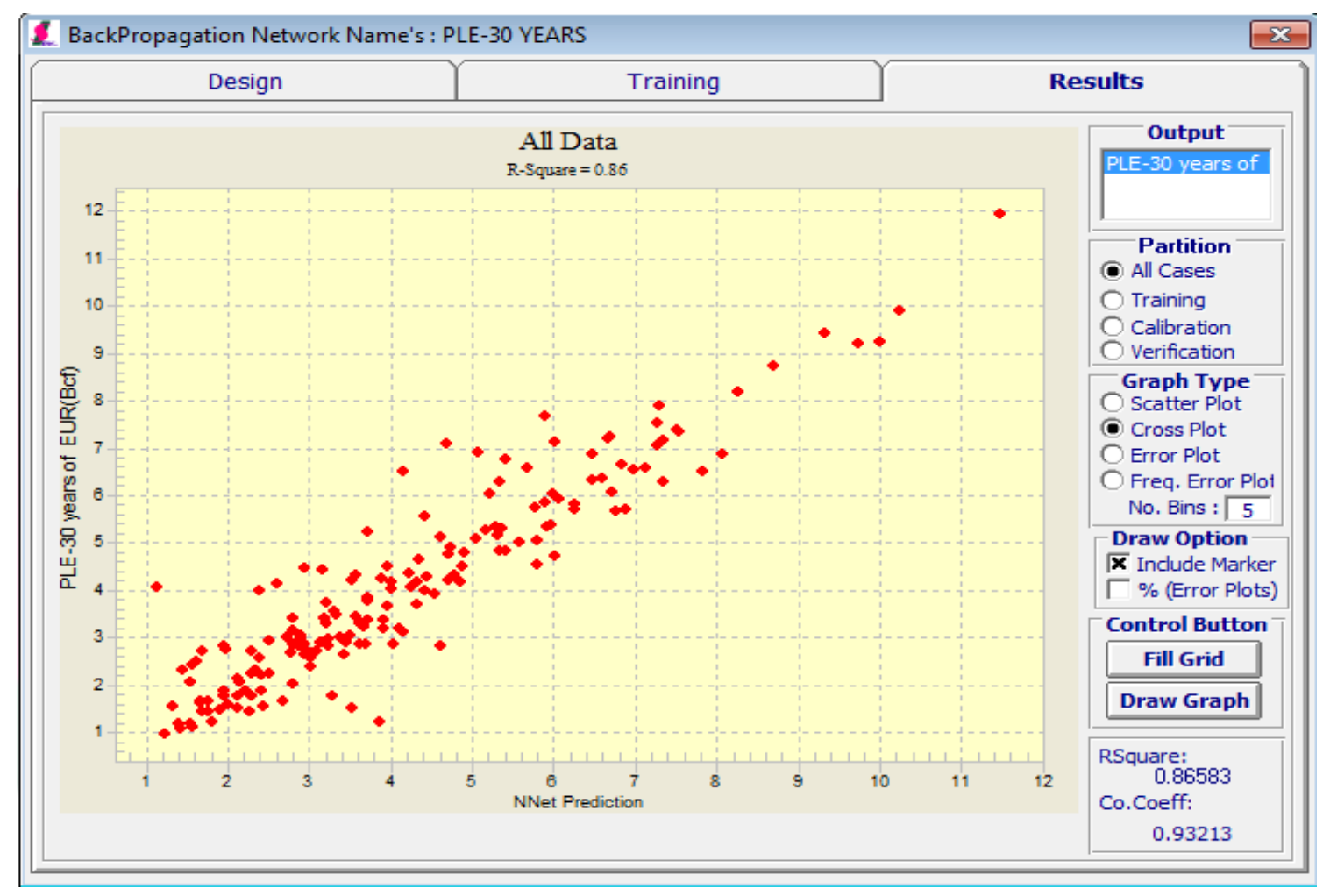

Figure 17: Backpropagation result for the trained NN-All Cases- Scatter Plot

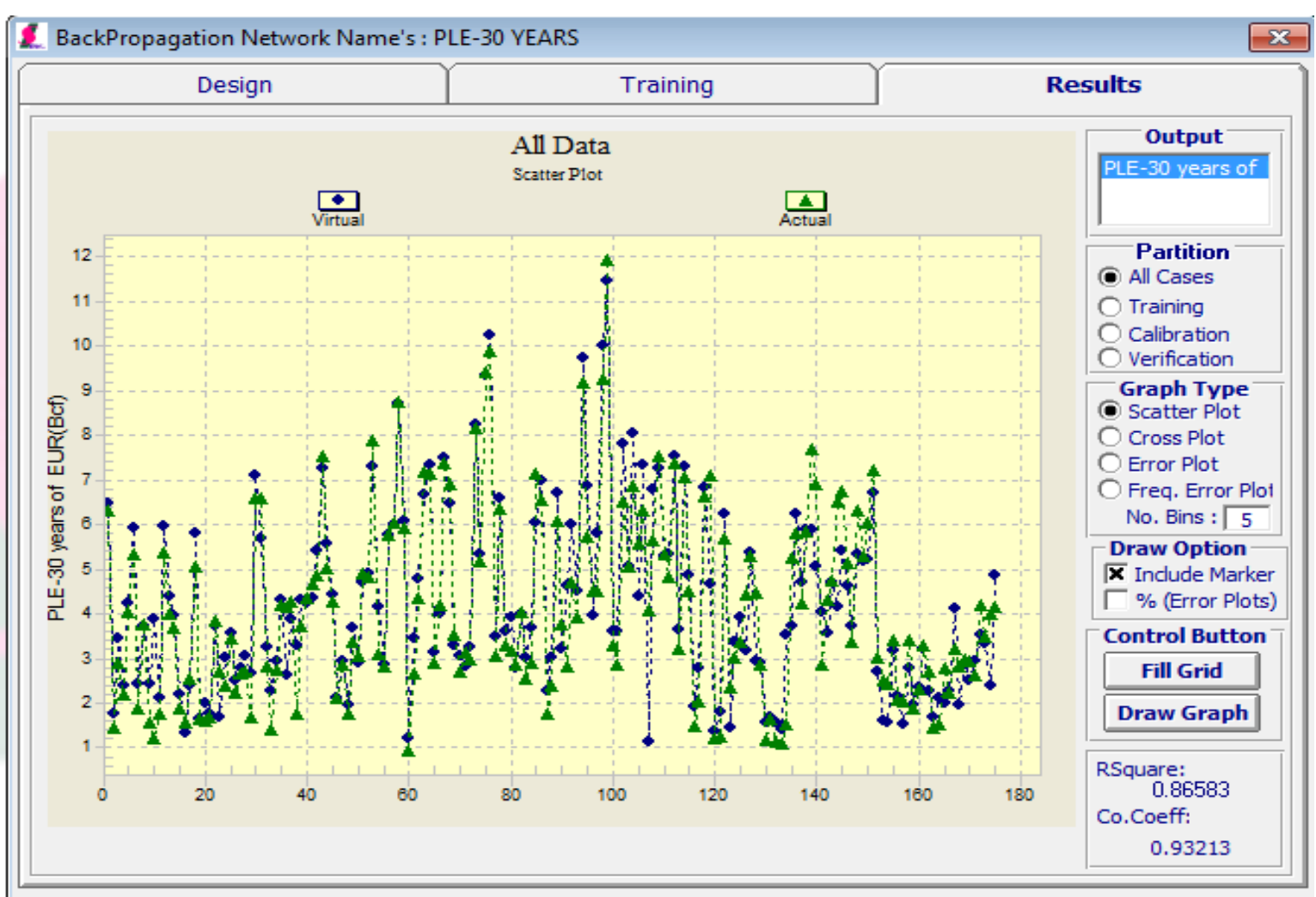

Figure 18: Backpropagation result for the trained NN-All Cases- Scatter Plot 


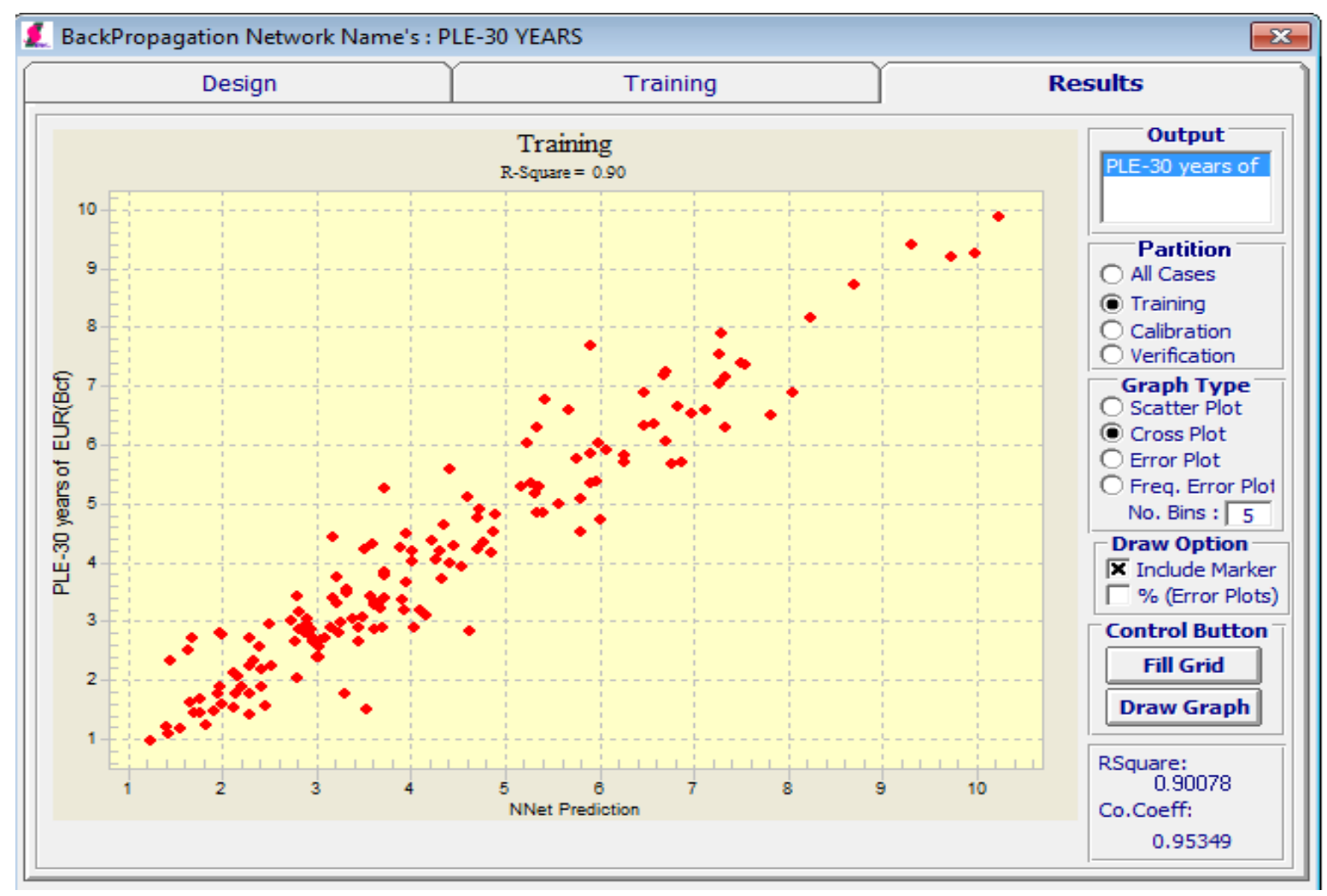

Figure 19: The impact of well location (Easting) on EUR for the models TED, PLE, SEPD and

HB- FTA result

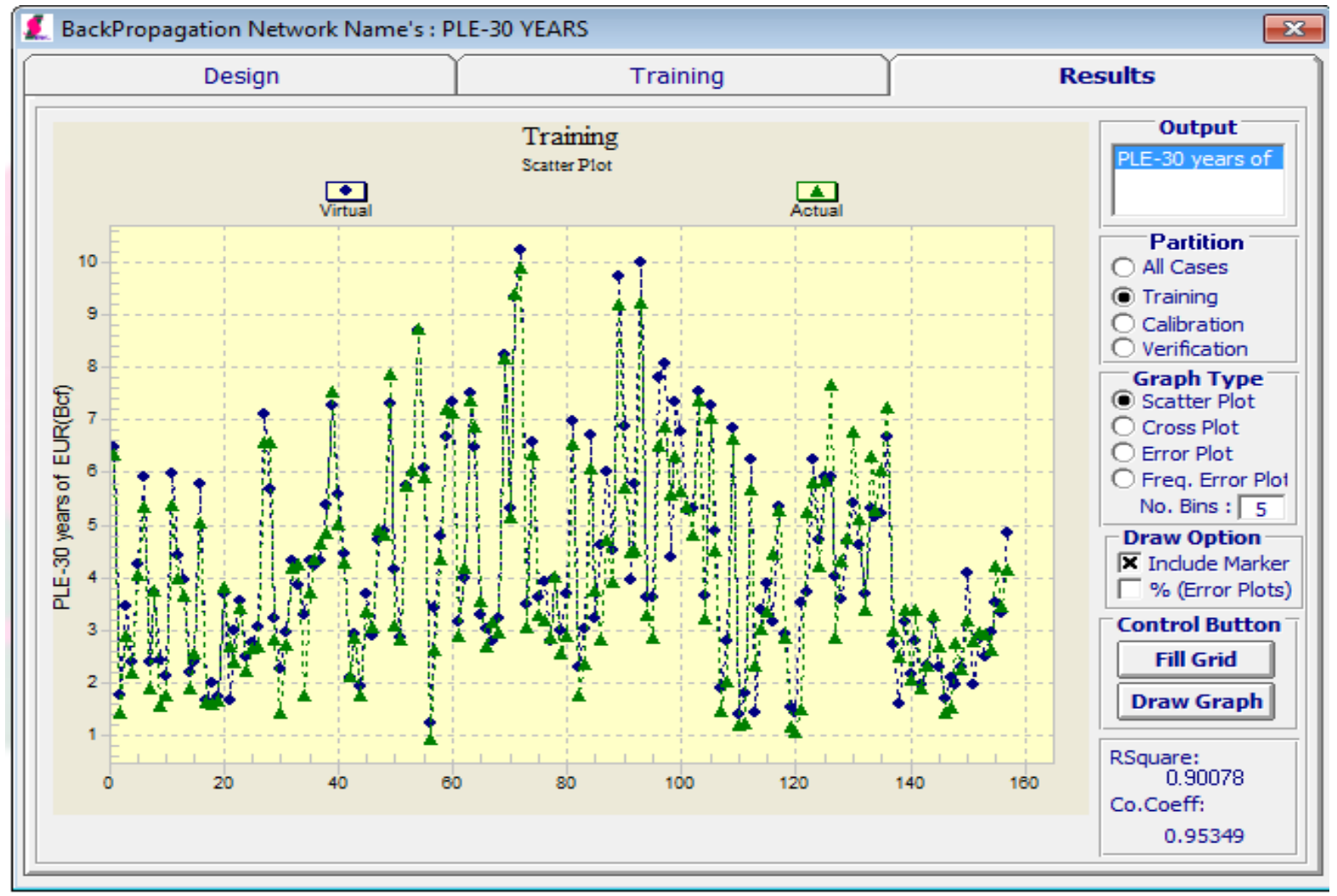

Figure 20: Backpropagation result for the trained NN-Training Data -Scatter Plot 


\subsubsection{Fuzzy Pattern Recognition analyses}

Extracting useful information or finding relation from large datasets of shale gas reservoirs by using same traditional or conventional tools is difficult tasks because of the complexities in petrophysical and mechanical properties of these reservoirs. Alternatively, in this study, the Fuzzy Pattern Recognition technique has been employed to find any desired hidden patterns among the complex dataset that described by non-linear relations.

Fuzzy Pattern Recognition (FPR) is unique and proprietary algorithms tool that is capable of deducing hidden and non-obvious trends and patterns from large complex field datasets. FPR is a branch of artificial intelligence concerned with classification the datasets and it derives from the concept of fuzzy theory. This tool is very widely used in industries because the experiences show that it discovers realistic relations among datasets.

In this study, Well Quality Analysis (WQA), and Fuzzy Trend Analysis (FTA) which are branches of Fuzzy Pattern Recognition (FPR) were applied to generate relevant and reasonable trends between EUR and parameters of reservoirs and completion design. In other words, WQA and FTA are employed to find the impact of these parameters on EUR.

Well Quality Analysis is process of Fuzzy Pattern Recognition but it is applied to a limited number of classes of wells such as Poor, Average, and Good Wells. In other hand, when every single well in the dataset is treated as a potential unique well quality, the result is called Fuzzy Trend Analysis.

Before showing the results of this study, it was important to present Conventional Statistical Analysis and why preferred to avoid using it in shale gas reservoirs. Figure (21) is demonstrating analysis for porosity in Cartesian, Semi-log, Log-Log and histogram. 


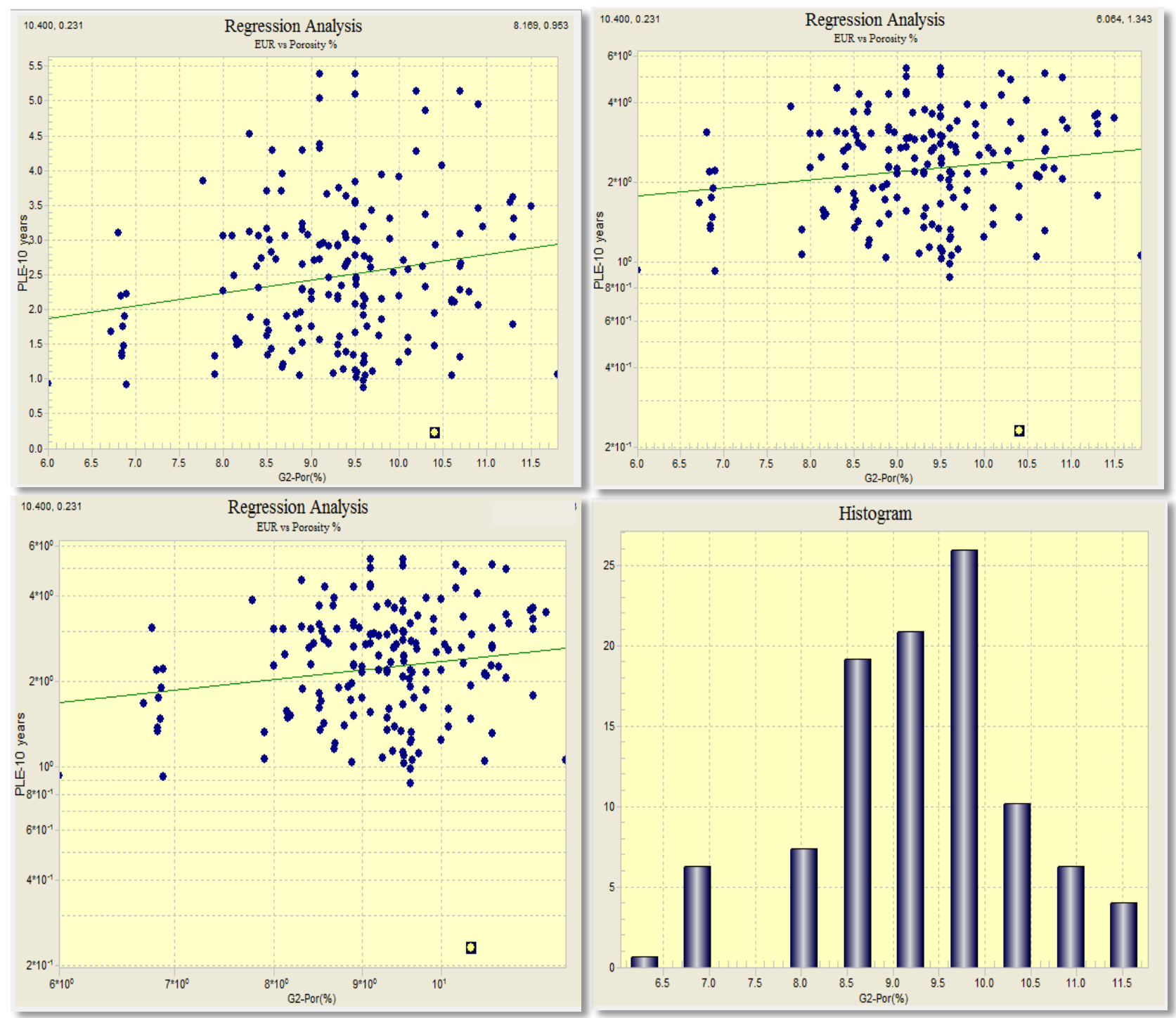

Figure 21: Conventional Statistical Analysis

In figure (21), the conventional statistical methods were used to show their weakness in extracting the trends between data for shale reservoirs. From these results, it can be seen that the conventional statistical was unsuccessful in finding trends or relation between the matrix porosity and EUR. However, it is very obvious that the higher porosity results better production performance and consequently results high EUR, which cannot be recognized by using conventional statistical analysis. In other hand, the Fuzzy Pattern Recognition (FPR) was able to extract the hidden pattern from the dataset. The Fuzzy Trend Analysis (FTA) in figure (22) and Well Quality Analysis(WQA) in figure (23) show the trend or relation between matrix porosity and EUR. 


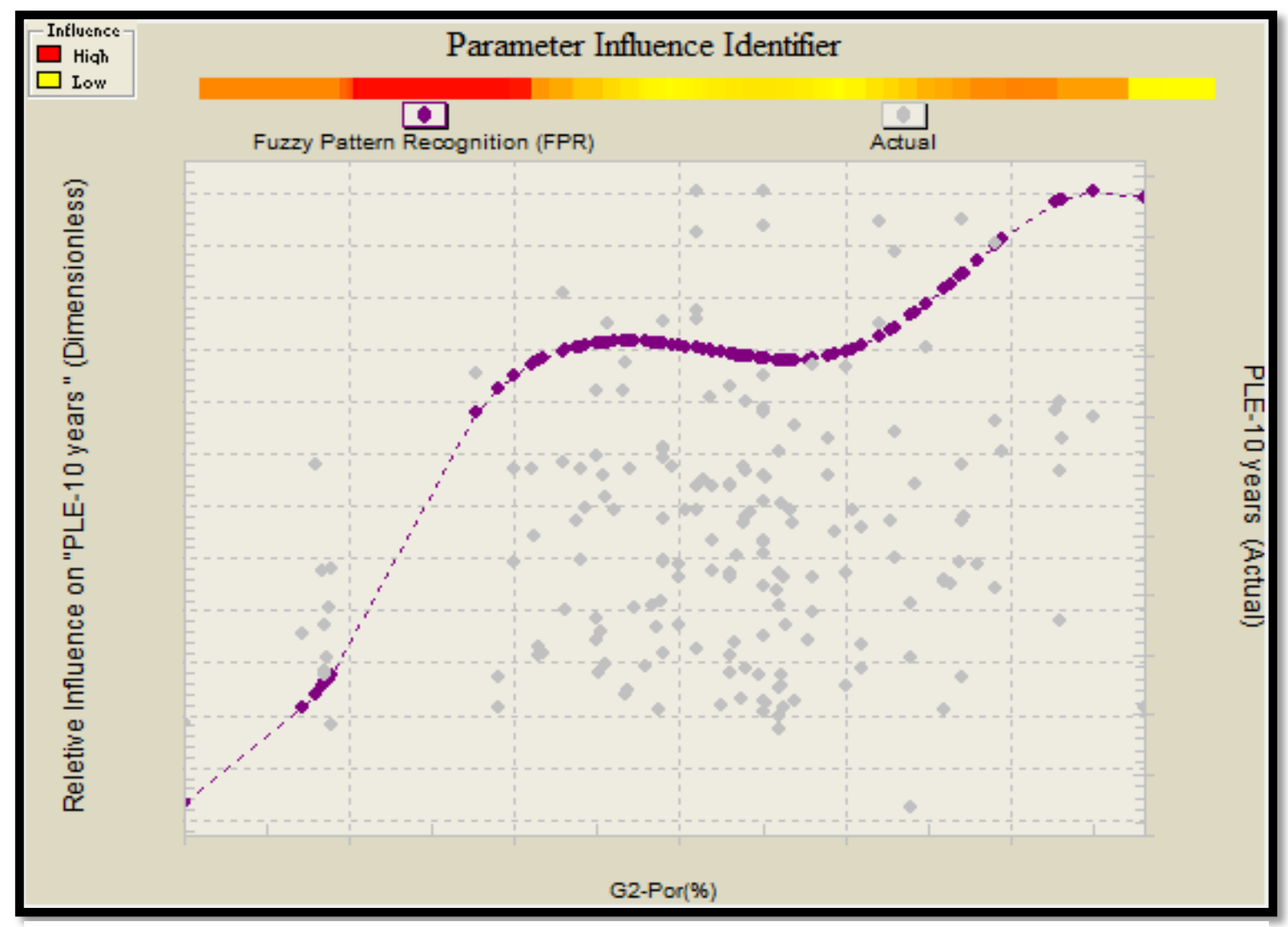

Figure 22: Fuzzy Trend Recognition- 10 years PLE EUR vs. \% Porosity

It can be seen from the above figure (22) that the EUR increase with high value of porosity which is very basic knowledge in reservoir engineering but it can not be found in conventional statistical analysis. In this figure (22) the purple points (or line) and the grey points represent the FTR and actual data respectively. The FTA extracted positive correlation between porosity and EUR.

Next figure (23) shows using WQA technique to classify the data based on EUR. In this figure, the wells have been divided into three groups which are poor, average and good porosity. The wells with good porosity give high EUR 


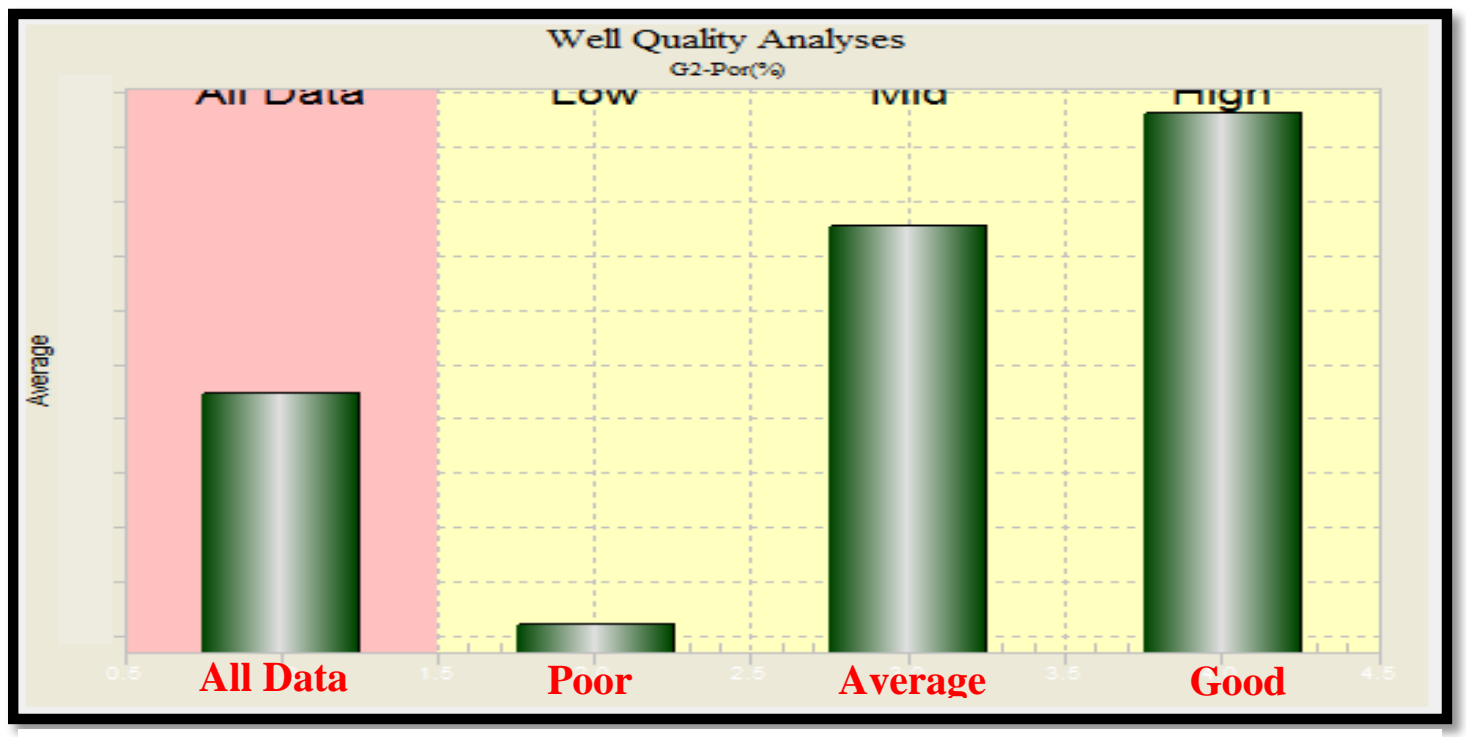

Figure 23: Well Quality Analysis based on porosity and 10 years PLE EUR

\subsubsection{Generating Type Curve}

The developed Models that have been trained by using IMprove Software in this study can be utilized to generate Type Curve. The Type Curve is a graphical technique used to show the impact of two parameters on EUR. In other words, the Type Curve makes a relation between two parameters and EUR by generating a set of curves which display the model behavior when the two parameters are changed. This relation can be employed to assist engineers during the decisionmaking.

In this type curve, the y-axis represent one of the output of the model such as EUR by PLE, SPED, HB or TED. In other side, $\mathrm{x}$-axis is one from the input parameters while the type curve is represented a third parameter from the input parameters. However, all the other input parameters are kept constant as average value of the parameter for all wells.

The evaluation and insight from the Type Curve can be used for optimization in completion plans, future drilling plans and stimulation plans. In addition, it also can be utilized to predict EUR for new wells that have not been drilled yet. For example, the Type Curve in figure() shows useful correlation between EUR, Measured Depth and Porosity. 


\section{Chapter 5: Results and Discussions}

In this section, the results of Fuzzy Trend Analysis (FTA) and Well Quality Analysis (WQA) will be discussed. Both FTA and WQA are employed to discover any hidden patterns in the datasets consequently detected the impacts of the parameters on EUR. In addition, important results were found in this research regarding whether reservoir or completion parameters have the most impact on EUR. This study was applied on more than 200 shale wells located in southwestern Pennsylvania. The same analysis has been performed on other parameters as well and the results are illustrated in Appendix A.

In order to perform the rigorous analysis presented in this study, the Estimated Ultimate Recovery are predicted for 10, 30 and 50 years by using the four techniques (PLE, SPED, HB AND TED). By applying these four techniques to the actual data from more than 200 shale wells, this study will take into account various parameters to insure more comprehensive results for the study.

IMPORTANT NOTE: The exclusive data of this study will not be published in these results. The data will be retained for private use only. 


\subsection{Total Organic Carbon (TOC)}

Total organic carbon (TOC) is one of main parameters to evaluate the potential of shale gas resources. The average TOC of prospective resevoir needs to be greater than $2 \%$. It is commonly believed to be true that a higher TOC value implies a greater production potentional for natural gas and oil. However, this positive correlation cannot be confirmed when considering the actual data which is represented by the grey dots which are the results of the conventional statistical analysis are used in shale gas reservoirs as noted in figure (24). In contrast, the red dots-line represents the Fuzzy Trend Recognition which successfully demonstrates the positive correlation between TOC and greater production potential for natural gas and oil.

Eventhough all of the four techniques of determining EUR ( see figure 24 ) show the same behavior in which EUR is greater when TOC has a higher percentage, the impact of TOC on EUR shows different individual results for each technique. For example, from the figure (24), formation with 3\% TOC has a EUR of 2.1 Bcf by using TED, 4.2 Bcf by using PLE, 5.2 Bcf by using SPED and 5.7 Bcf by using HB. Nevertheless, TED model present more reasonable trend comparing to other models.

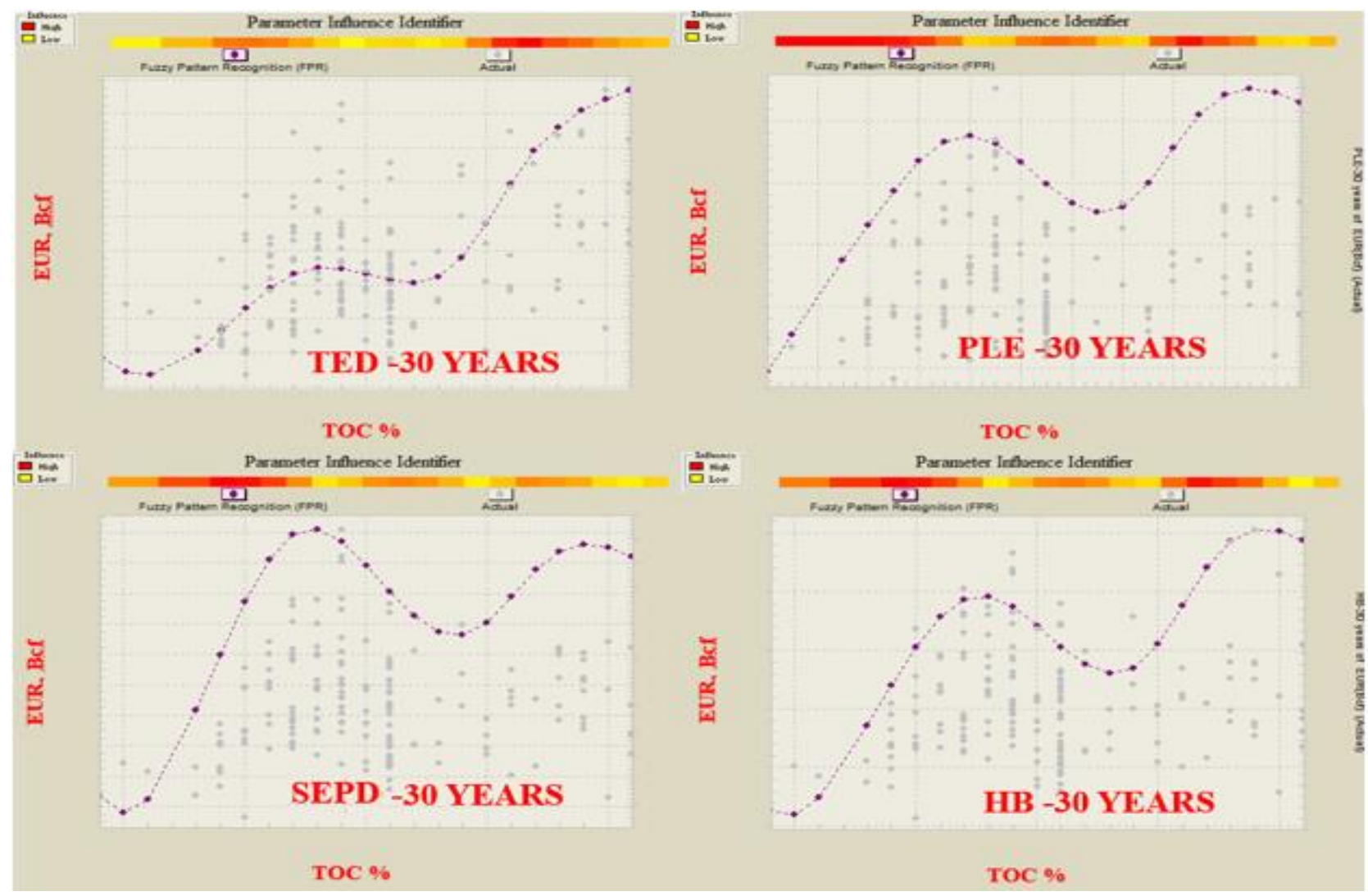

Figure 24: The impact of TOC on EUR for the models TED, PLE, SEPD and HB- FTA result 


\subsection{Young's Modulus}

Young's Modulus is defined as the resistance of rock to elastic deformation under pressure. It is one of the most important parameters that should be considered to achieve fracking efficiency. In general, the rocks with less young's modulus mean more flexible rock and consequently are fractured easily.

Figure (25) shows the impact of Young's modulus on EUR in the four techniques that used to calculate EUR. Based on FTA in this figure, the FTA trend reveals that the wells with higher young's modulus show lower EUR than these with lower young's modulus. Further, PLE model is showing more reasonable trend than the other models.

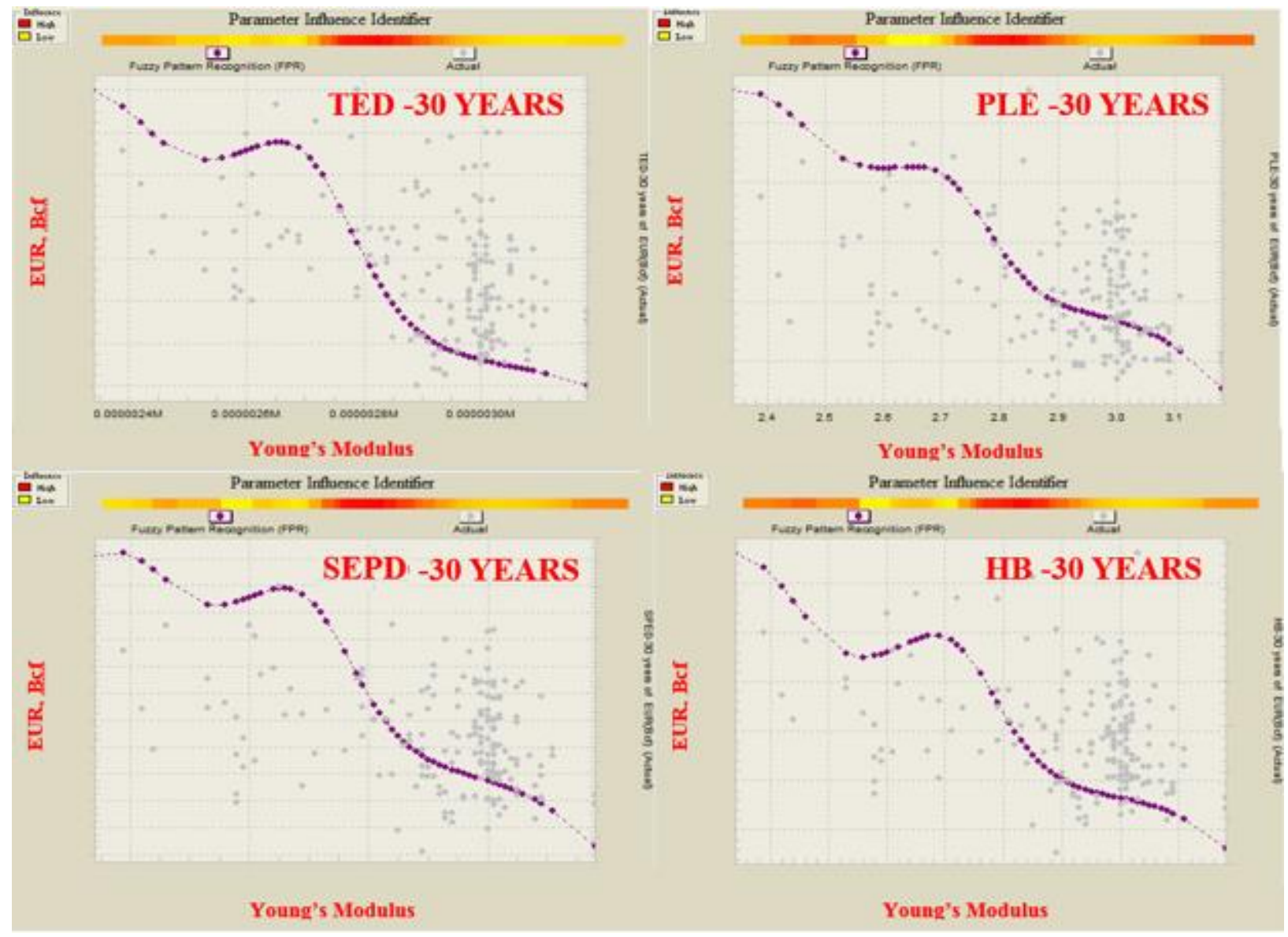

Figure 25: The impact of Young's Modulus on EUR for the models TED, PLE, SEPD and HBFTA result 


\subsection{Stimulated Lateral Length}

It is accepted to be true that drilling a horizontal well with a long lateral length will provide a greater ultimate production. In figure (26), the FTA extracts this positive correlation in Marcellus shale wells dataset for all the four techniques of EUR. However, The PLE and SPED show reasonable trend when combined to other techniques.

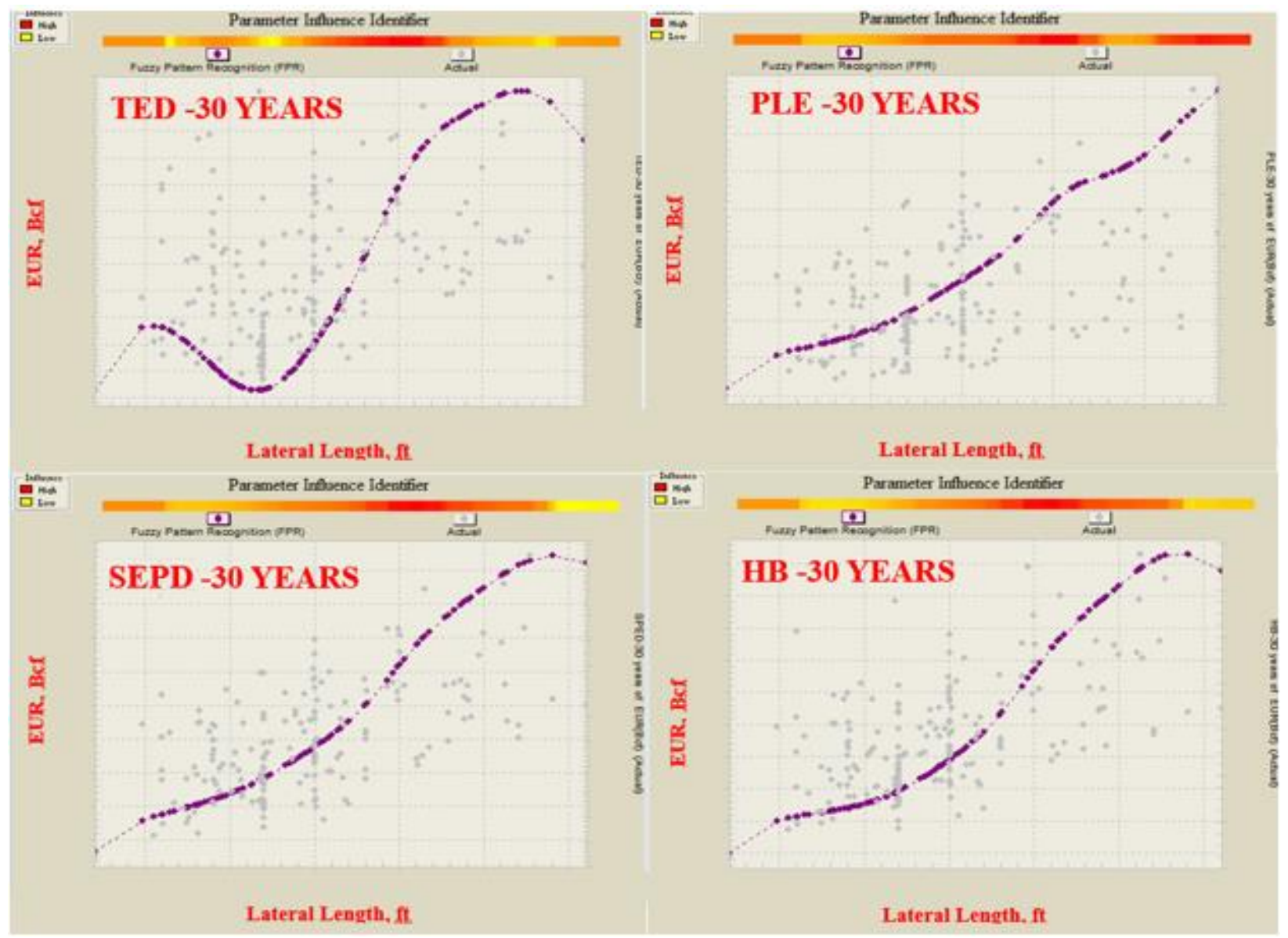

Figure 26: The impact of Stimulated Lateral Length on EUR for the models TED, PLE, SEPD and HB- FTA result 


\subsection{Total Number of Stages}

The actual data (grey dots) was scattered and show no correlation between EUR and number of stages. FTA (see red dots- curve in figure 27) clearly shows that wells provide a higher EUR when completion is designed with more number of stages. However, this is not a new conclusion in petroleum engineering because it has been already proved that productions behavior increase with more number of stages. This study has proved that the impact of increasing number of stages on EUR shows different results for each technique of determining EUR. In addition, it is observed that TED technique was not very good in showing this obvious correlation.

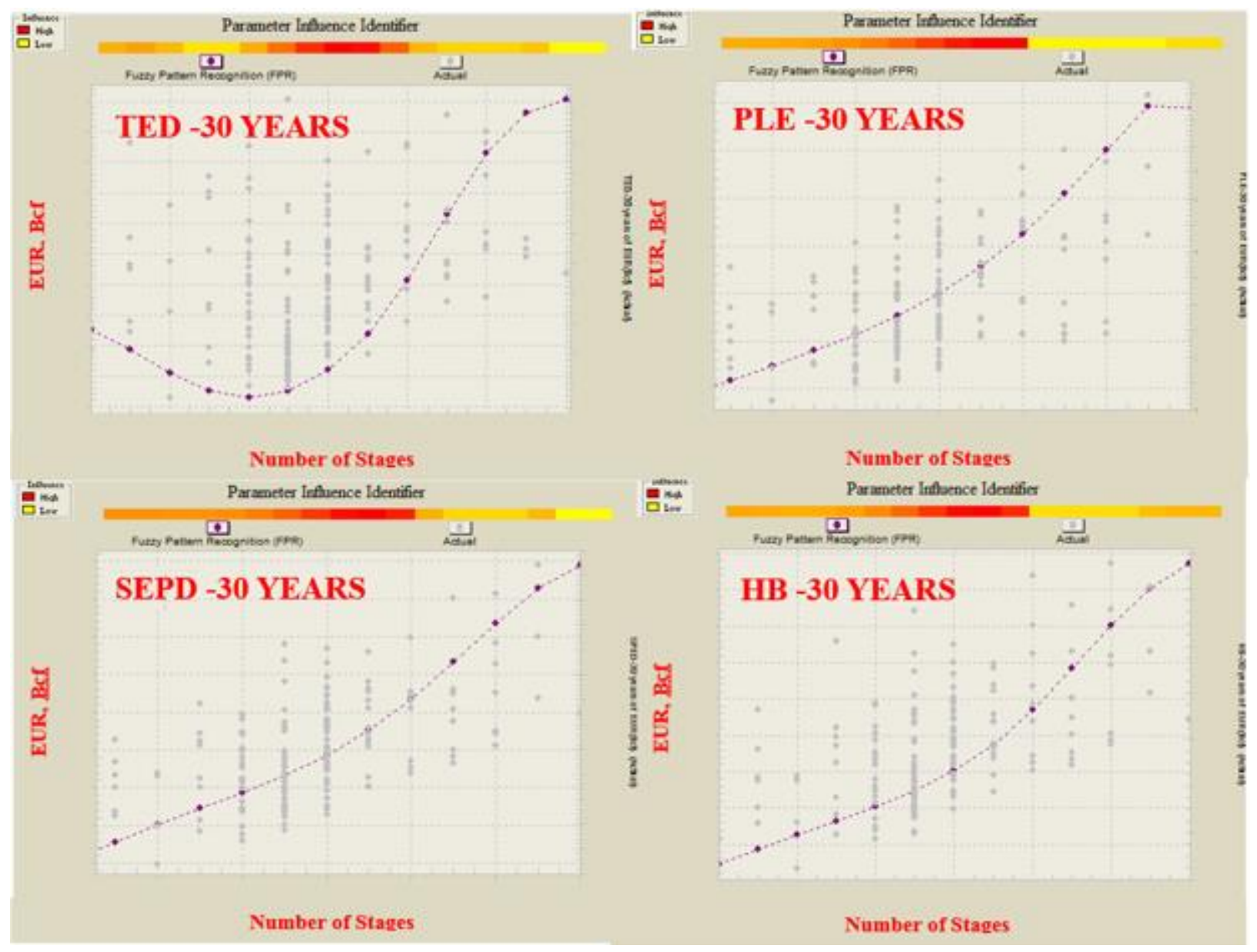

Figure 27: The impact of Number of Stages on EUR for the models TED, PLE, SEPD and HBFTA result 


\subsection{Cluster Spacing}

It can be seen from figures (28) and (29) that when the completion has been designed with close cluster spacing, the well will provide higher EUR than this well with large cluster spacing. The FTA and WQA present this result in figures (28) and (29) respectively. The main reason is that reducing the cluster spacing will increase the conductive transverse fractures.

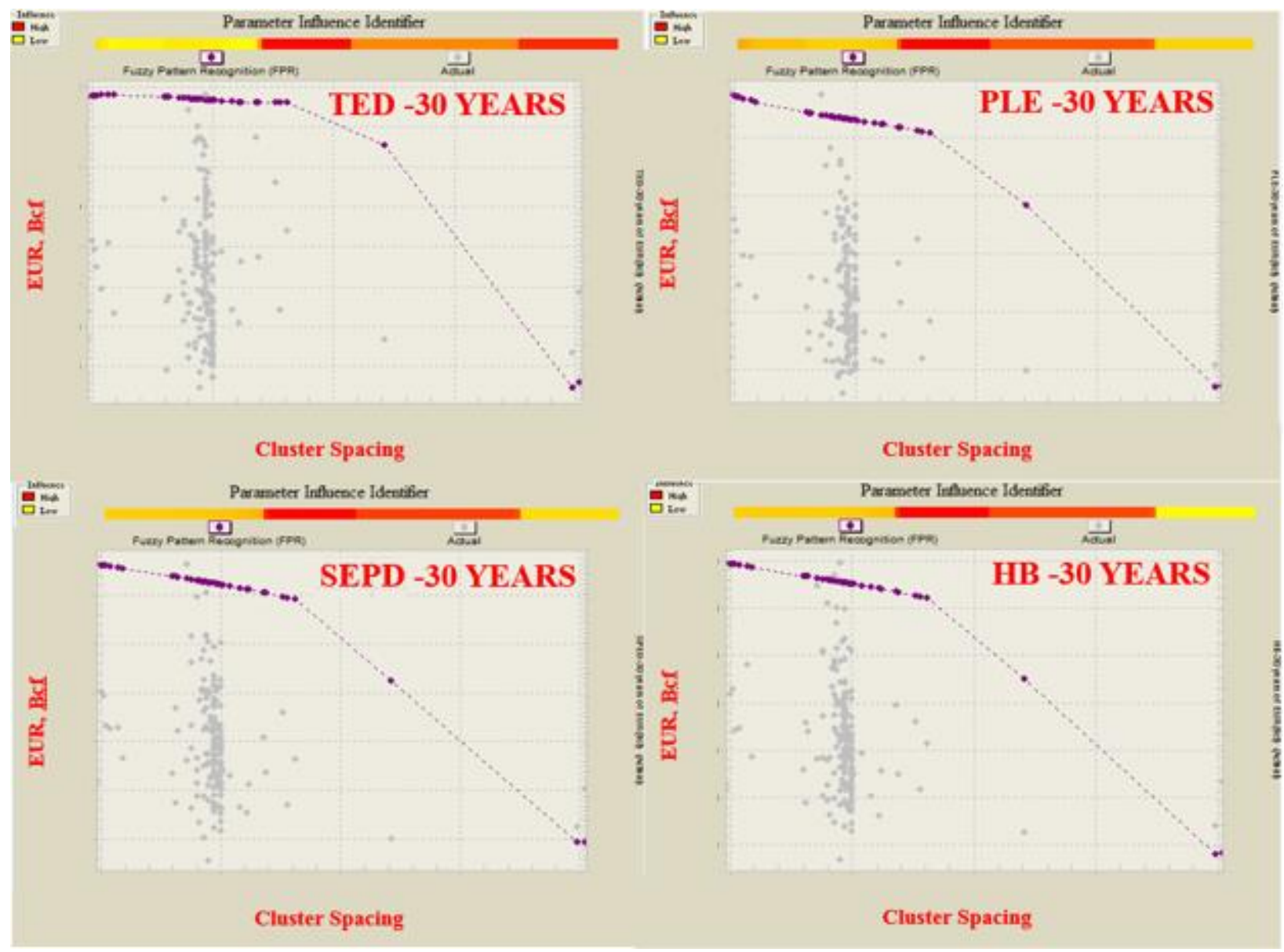

Figure 28: The impact of Cluster Spacing on EUR for the models TED, PLE, SEPD and HBFTA result 


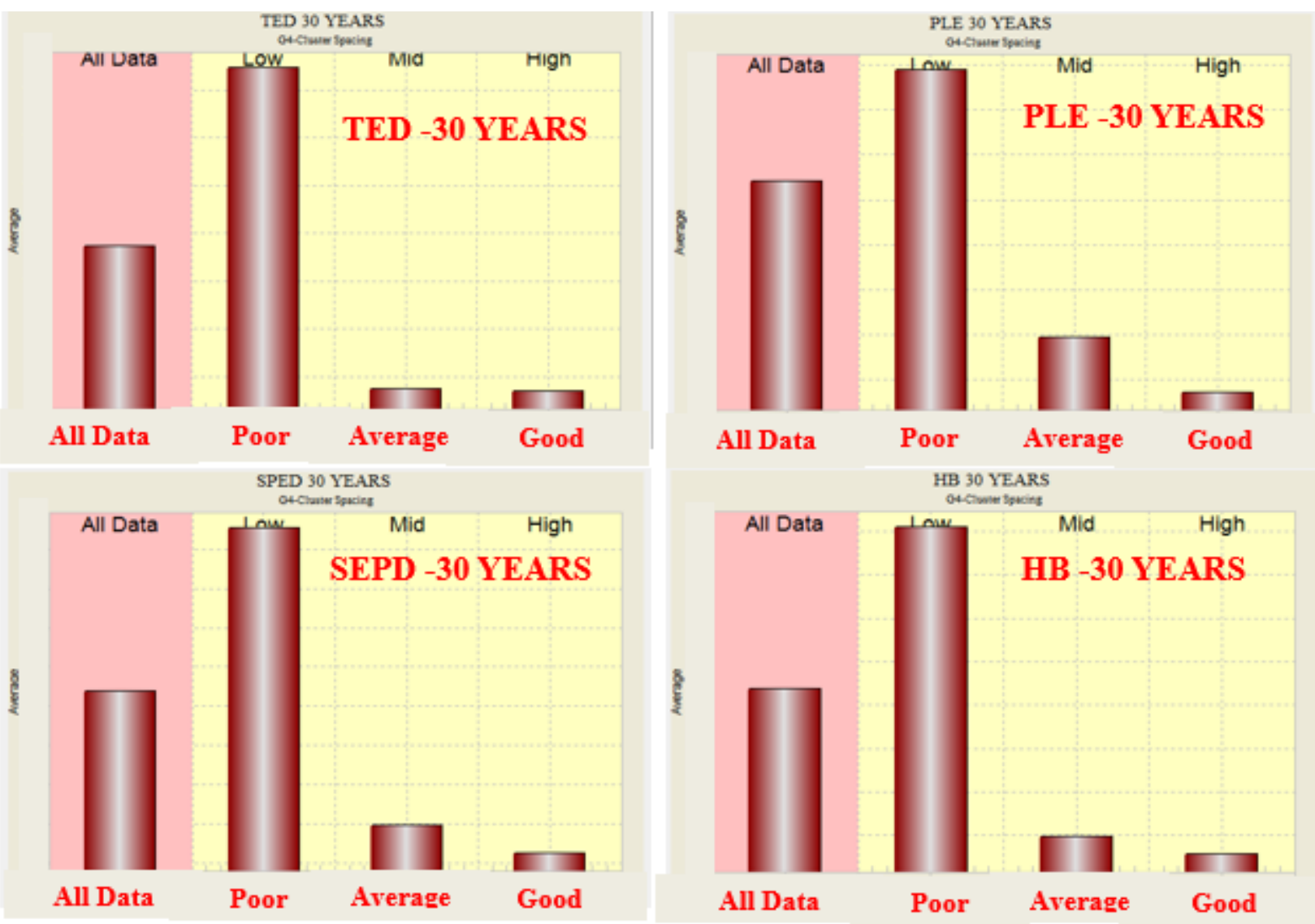

Figure 29: Well Quality Analysis of Cluster Spacing for models TED, PLE, SEPD and HB

\subsection{Injected Proppant per Stage}

A proppant is a solid material that pumped to the formation during or following a fracturing treatment to keep the hydraulic fracture open. Both FTA and QWA, in figure (30) and figure(31), show that treating the wells with more proppant in hydraulic fracturing results high EUR. It can be seen from figure (30) that FTA was more reasonable in TED than the other techniques.

In other side, the WQA (see figure 31) was successful in showing this positive correlation in all the four methods. The high quality wells are those pumped with more proppant during the fracturing treatment. 


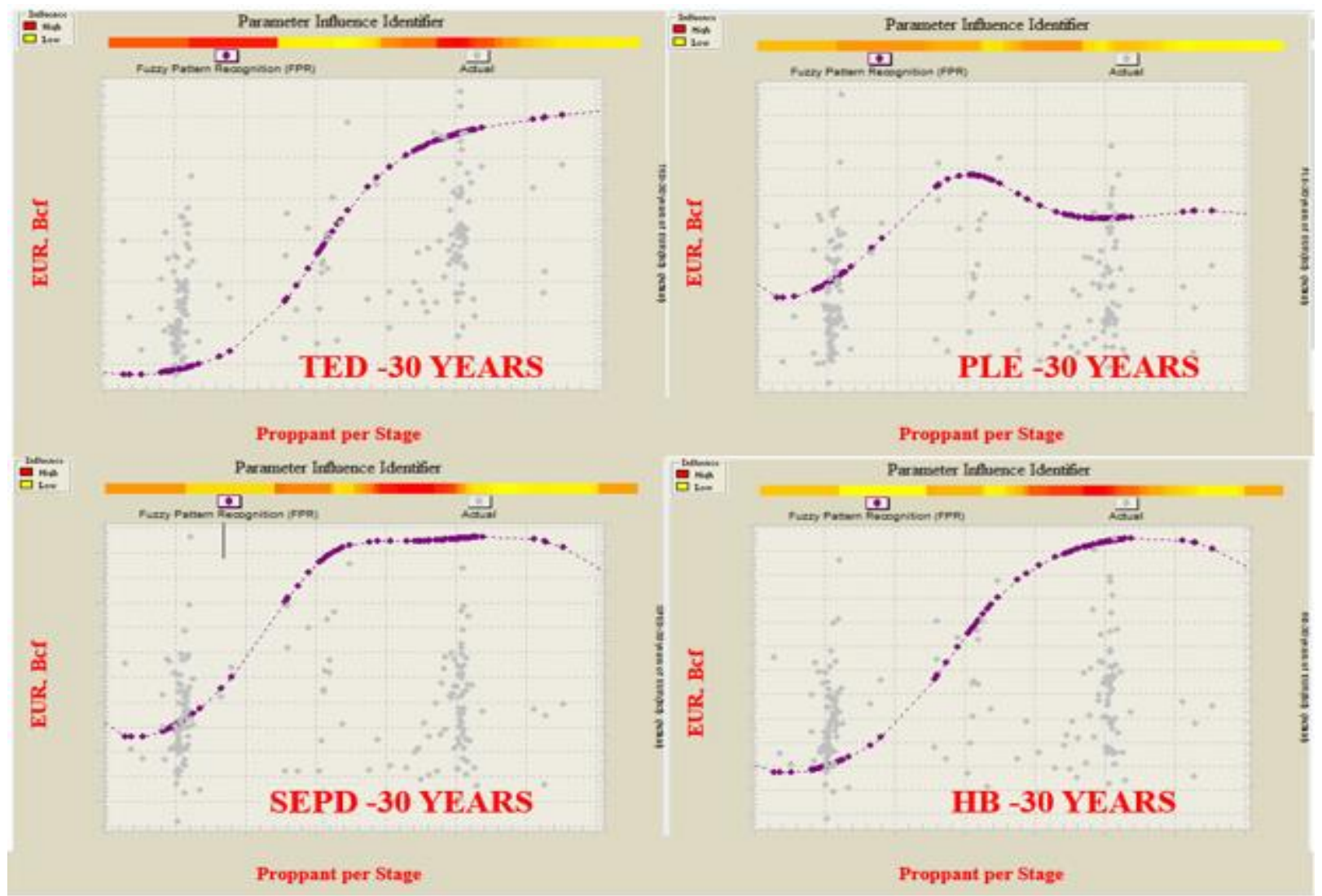

Figure 30: The impact of Injected Proppant per stage on EUR for the models TED, PLE, SEPD and HB- FTA

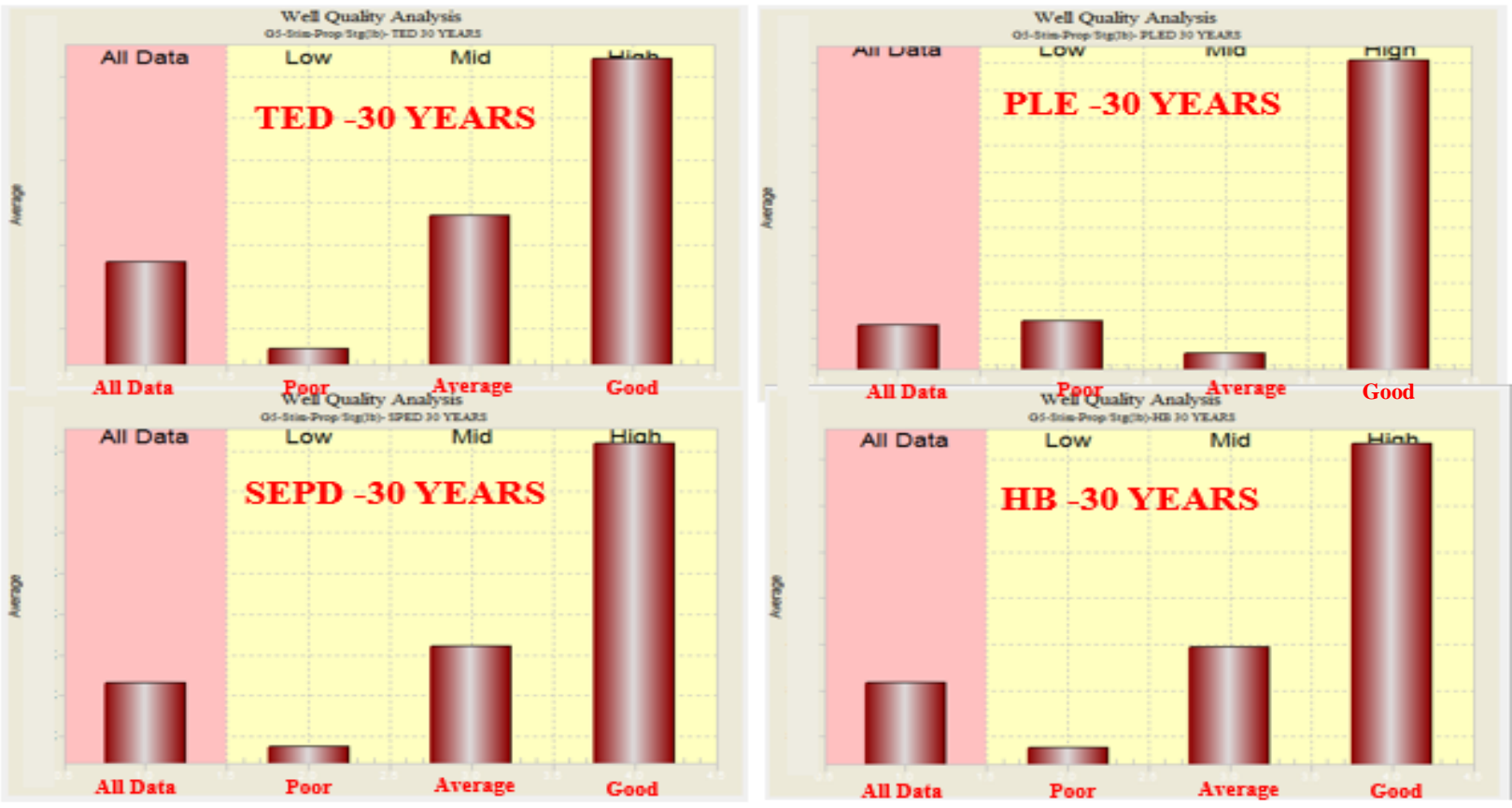

Figure 31: Well Quality Analysis of Injected Proppant per Stage for EUR models TED, PLE, SEPD and HB 


\subsection{Soak Time}

Soak Time is defined as the time between completion the well and when it is opened to production. This parameter plays very important rule in the well life. From FTA (figure 32), it is noted that high EUR results when the well has less soak time. Based on this, commencing production should begin with minimal delay after completion to improve the future production of the well. The reason behind this behavior was explained by [Holditch, 1979] that is injection water after hydraulic fracturing job may reduce the formation permeability in the invaded zone because the clay swelling, scaling and fines migration.

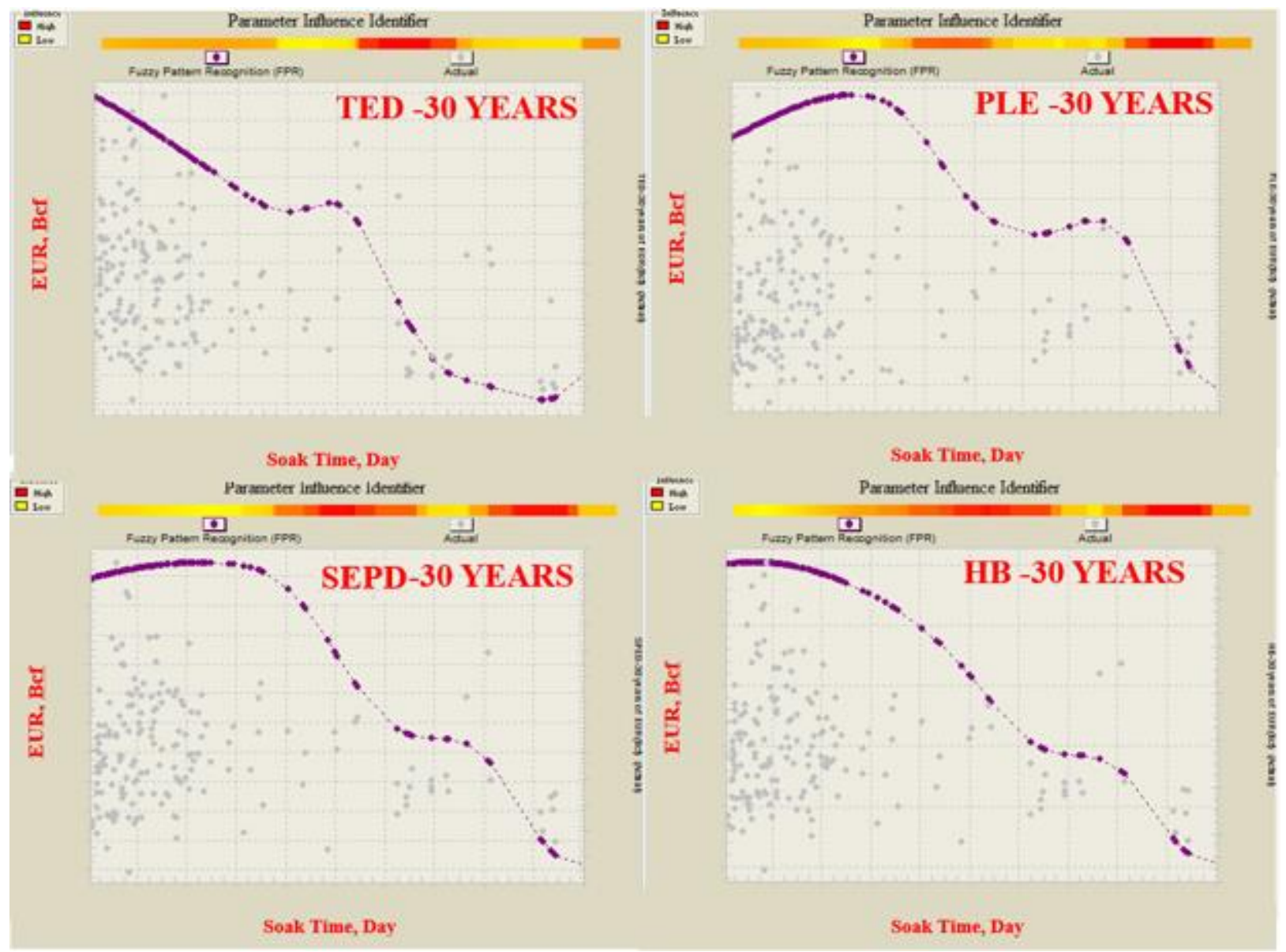

Figure 32: The impact of Soak Time on EUR for the models TED, PLE, SEPD and HB- FTA result

In addition, the WQA (see figure 33) confirms that wells with shorter soak time provide more EUR than these wells with longer soak time. The average soak time of these wells is different based on the technique that used to calculate EUR. However, all the techniques show that the same behavior of having a shorter soak time will improve the ultimate production of the well. 


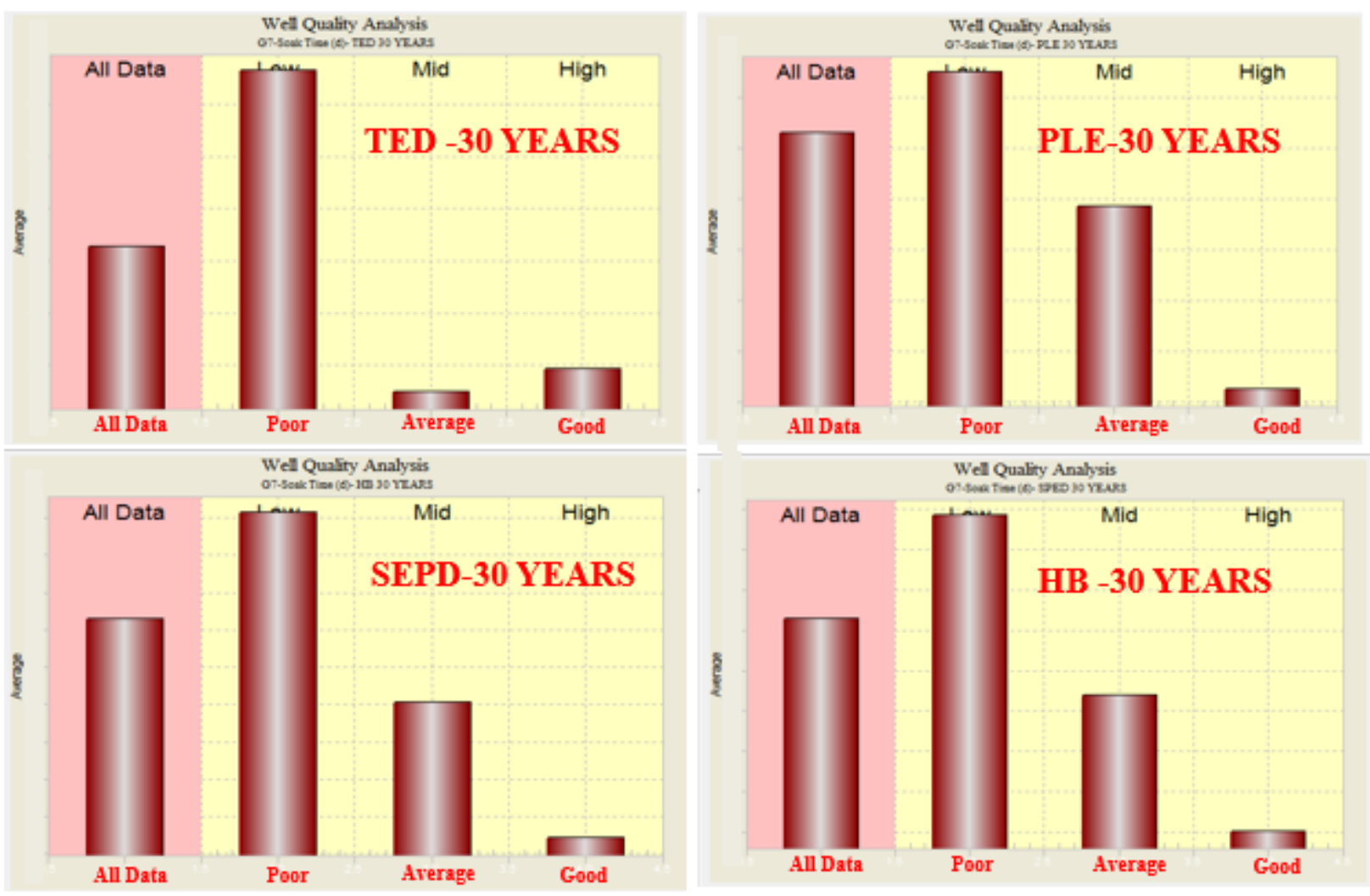

Figure 33: Well Quality Analysis of Soak Time for EUR models TED, PLE, SEPD and HB

\subsection{Thickness of Pay Zone:}

The thickness of the Marcellus Shale formation ranges from less than 5 feet thick to more than 250 feet thick. This thickness increases gradually from the eastern part of the Marcellus Shale formation to the western part. In general, by having greater thickness pay zone, the formation produces more. The FTR (figure 34) extracts this positive correlation by applying the four EUR techniques. It can be seen in this figure that PLE and SEPD show more reasonable trend than the other two models. In addition, it is observed that from the WQA (figure 35) that the wells with greater thickness produce more. 


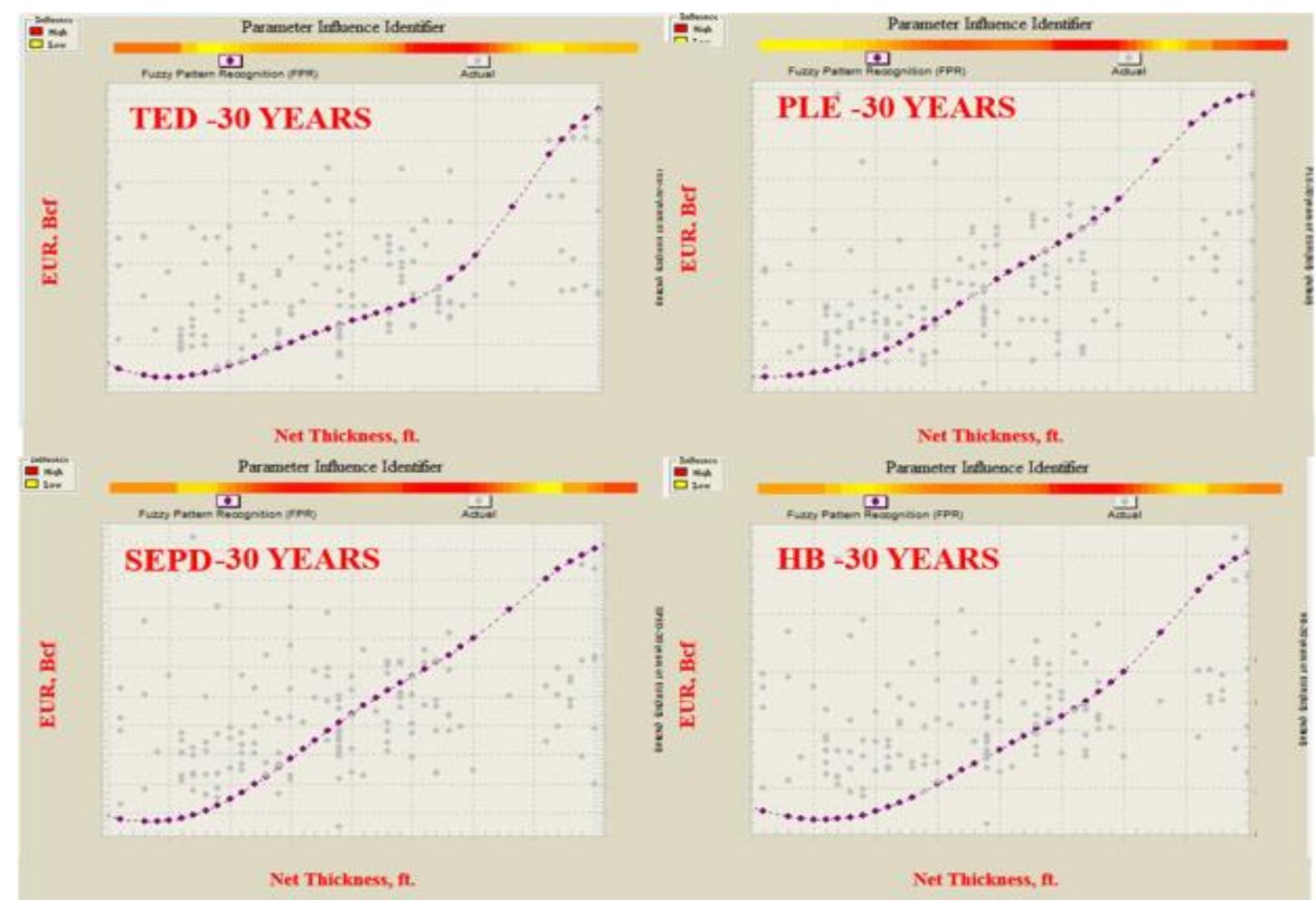

Figure 34: The impact of Net Thickness on EUR for the models TED, PLE, SEPD and HB- FTA result

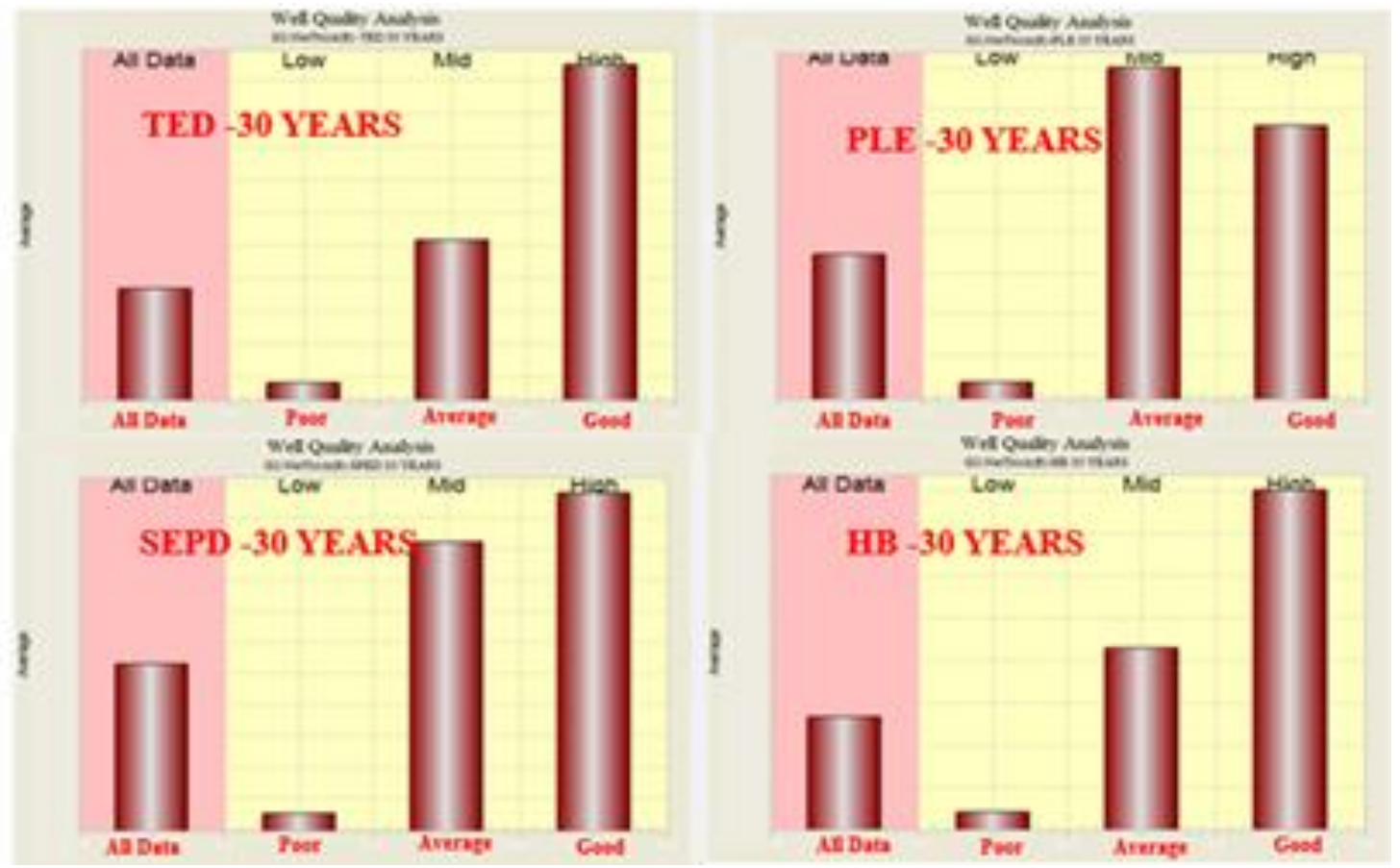

Figure 35: Well Quality Analysis of Net Thickness for EUR models TED, PLE, SEPD and HB 


\subsection{Matrix Porosity}

In Figure 36, gray dots represent the actual data and the red dots-line curve are the result of Fuzzy Trend Analysis (FTA) process. As mentioned before, the conventional statistical analysis cannot extract pattern between the input and output. In this figure, the gray dots represent the actual data scatter between $\mathrm{x}$-axis and $\mathrm{y}$-axis (by using conventional statistical analysis). However, the FTA, the red dots-line curve, finds reasonable trend that by increasing the matrix porosity, the future production will be increased. It has to mention that each method provides different result of EUR when applied to same matrix porosity.

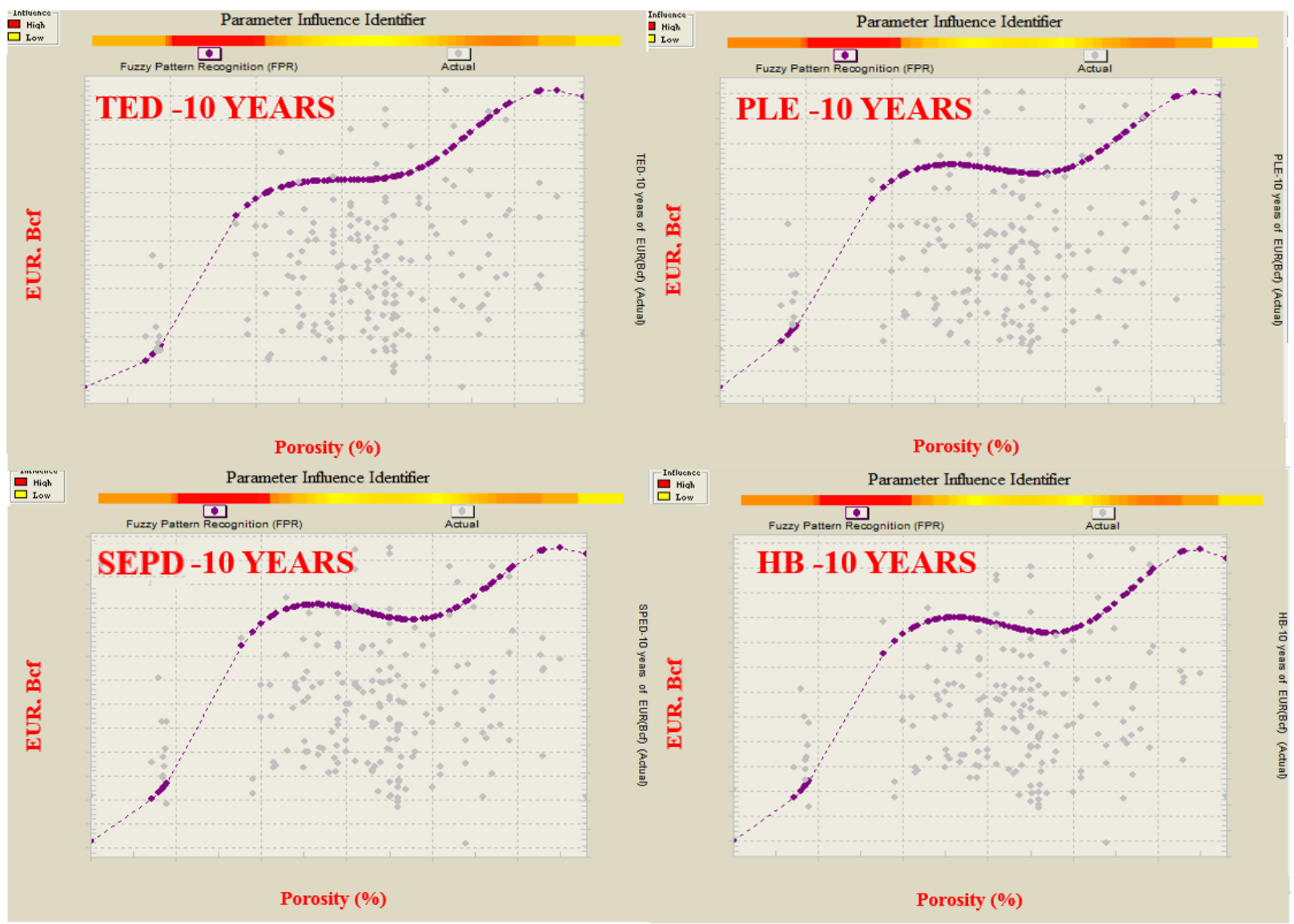

Figure 36: The impact of Matrix Porosity on EUR for the models TED, PLE, SEPD and HBFTA result 


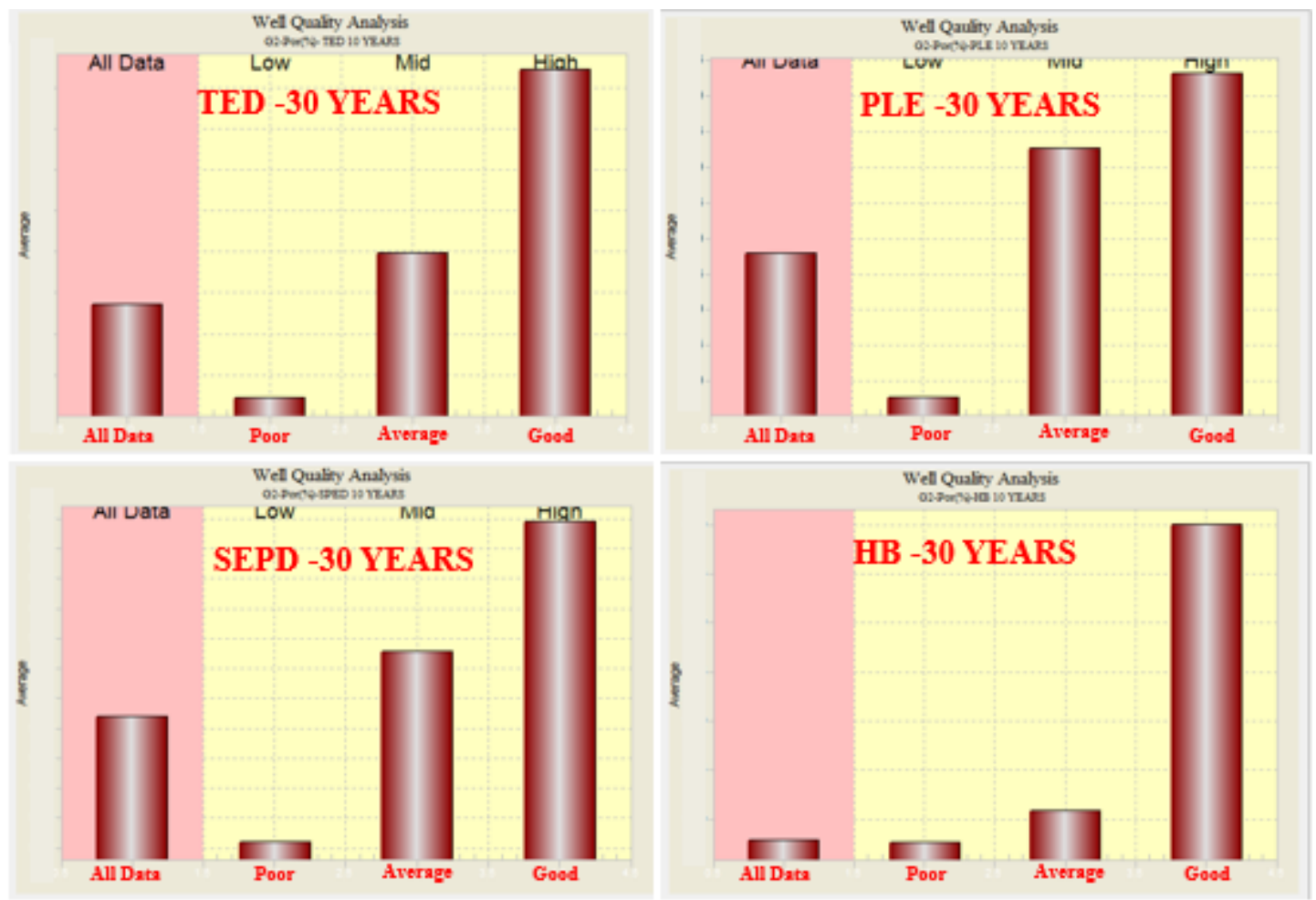

Figure 37: Well Quality Analysis of Matrix Porosity for EUR models TED, PLE, SEPD and HB 


\subsection{Generating Type Curve}

\subsubsection{EUR as a Function of Measured Depth and Porosity in Type Curve}

The Type Curve in figure (38) shows useful correlation between EUR, Measured Depth and Porosity. The Y-axis represents EUR that calculated by TED, PLE, SEPD and HB. While the Xaxis is the Measured Depth (MD) which is changing as a continuous parameter. The third parameter is the Porosity as different discrete values represented by the curves.

This Type Curve can be used to predict EUR for new wells that have not been drilled yet. For example, by drilling new well with $\mathrm{MD}=10000 \mathrm{ft}$., this will provide different values of EUR based on the porosity values of the formation. In this example, by using TED method, the 10 years of the EUR are $2.2 \mathrm{Bcf}$ for 0.06 porosity, 2.3Bcf for 0.0716 porosity, $2.45 \mathrm{Bcf}$ for 0.0832 porosity, $2.6 \mathrm{Bcf}$ for 0.0948 porosity, $2.74 \mathrm{Bcf}$ for 0.106 porosity and 2.88 for 0118 porosity. Different results will be provided when different model is used for same MD.
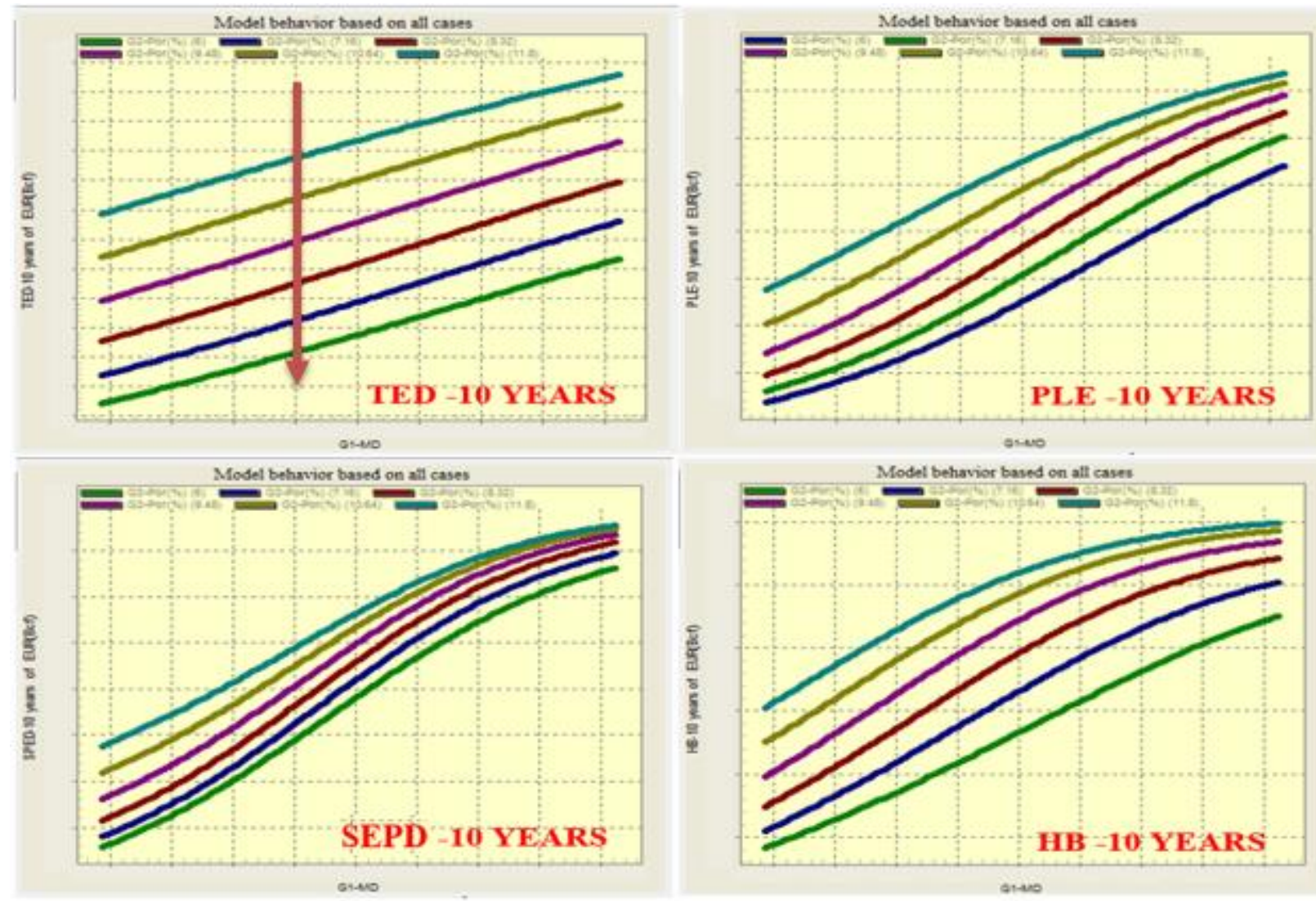

Figure 38: EUR as a Function of Measured Depth and Porosity in Type Curve 


\subsubsection{EUR as a Function of True Vertical Depth and Lateral Length in Type Curve}

Drilling a new horizontal well requires optimization the best lateral length that can be designed to enhance the production. In this Type Curve (see figure 39 ), different laterals length are suggested for each one True Vertical Depth (TVD). For example, by using PLE model, drilling well with TVD $6400 \mathrm{ft}$. and 150ft. lateral length will be estimated to produce $1.6 \mathrm{Bcf}$. While extended the lateral length to $4500 \mathrm{ft}$. will be estimated to produce 4.2 Bcf. However, it can be seen that the other models such as TED, SEPD and HB provide different results.
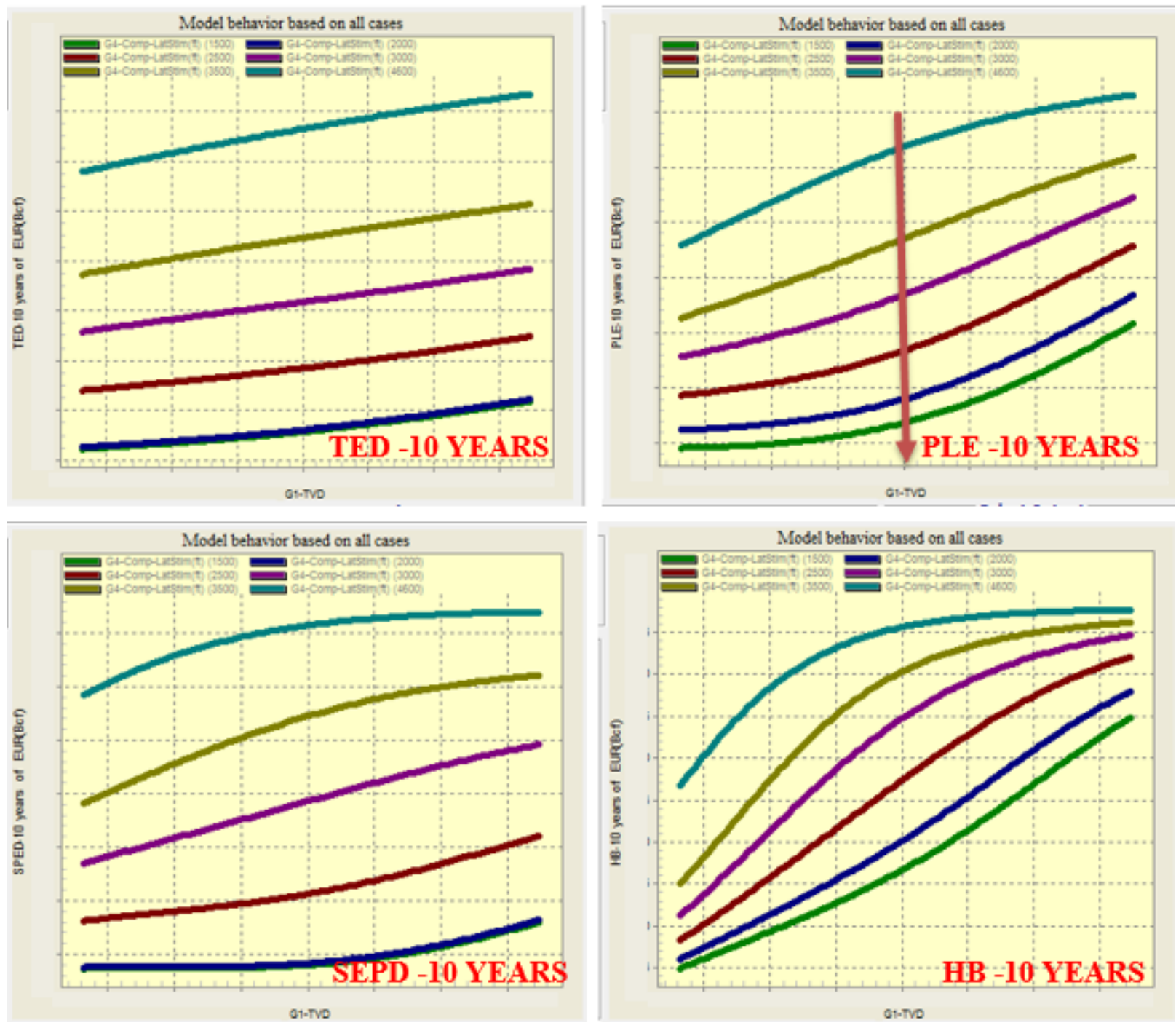

Figure 39: EUR as a Function of True Vertical Depth and Lateral Length in Type Curve 


\subsubsection{EUR as a Function of Lateral Length and Number of Stages in Type Curve}

It is very important to optimize the suitable number of stages for each lateral length in horizontal wells. Figure (40) shows very useful Type Curves that can be used to design the number of stage with each lateral length. Completion well with $3000 \mathrm{ft}$. lateral length and 4 stages will provide 1.5 Bcf for 10 years EUR based on TED model. By increasing the number of stages for the same lateral length, the well will be estimated to produce $1.8 \mathrm{Bcf}$ with 8 stages, $2.4 \mathrm{Bcf}$ with 12 stages and 3.2 Bcf with 22 stages. Though, the other models of EUR show different results.
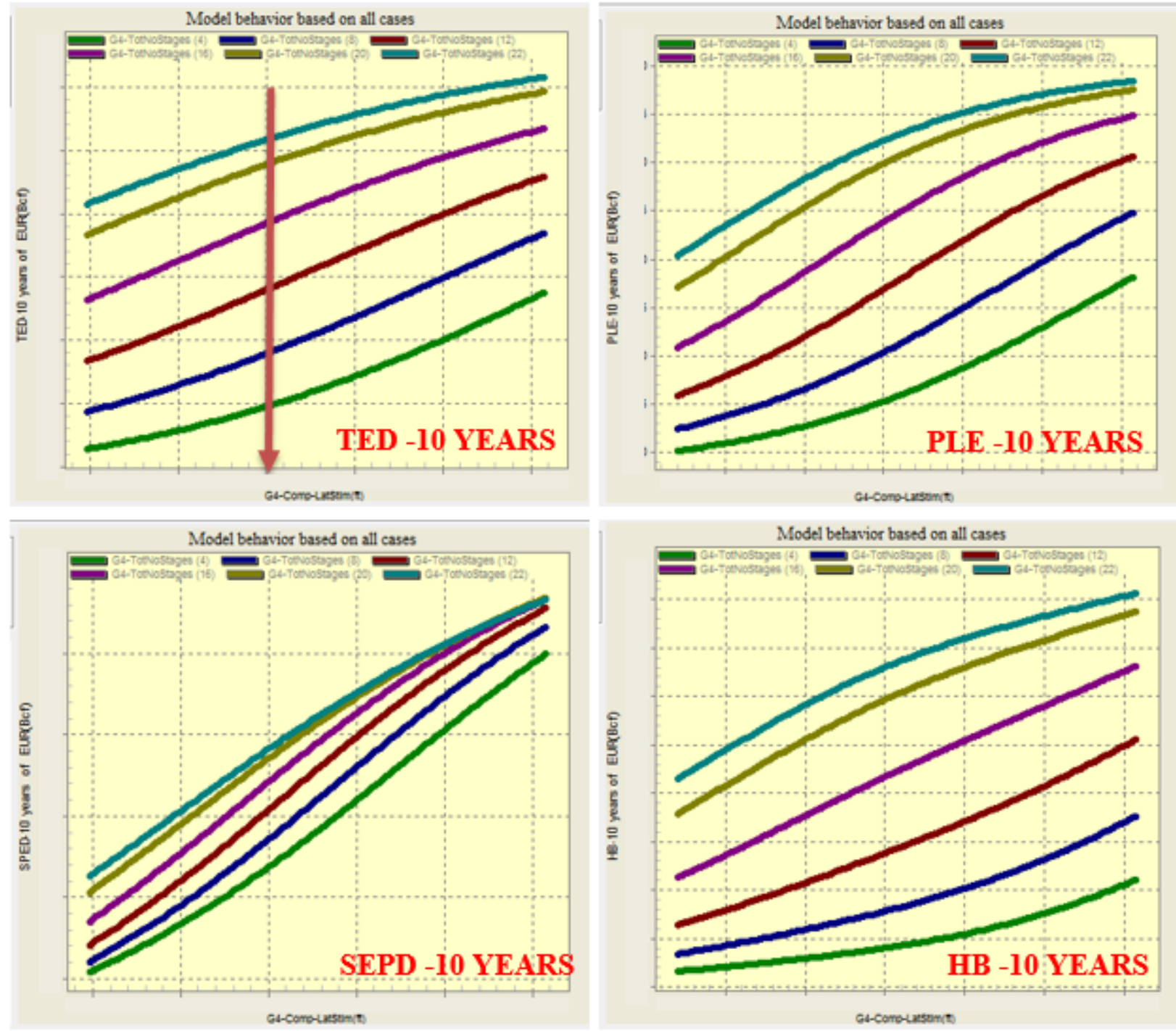

Figure 40: EUR as a Function of Lateral Length and Number of Stages in Type Curve 


\section{Chapter 6: Conclusions and Recommendations}

\subsection{Conclusions}

According to this study, the following results were found:

1) Figure (41) depicts the average influence of Native and Design Parameters on EUR. From this figure, critical facts were found regarding which parameters, Native (reservoir) or Design (completion), have the most impact on EUR. The results of EUR were significantly impacted by design parameters for all four models. However, Native parameters have the most influence on EUR with the TED model. Therefore, to emphasize the impact of the reservoir characteristics on EUR one needs to use the TED techniques and to emphasize the impact of the design characteristics one needs to use HB. Results generated by SEPD and PLE clearly fall between these two decline curve techniques.

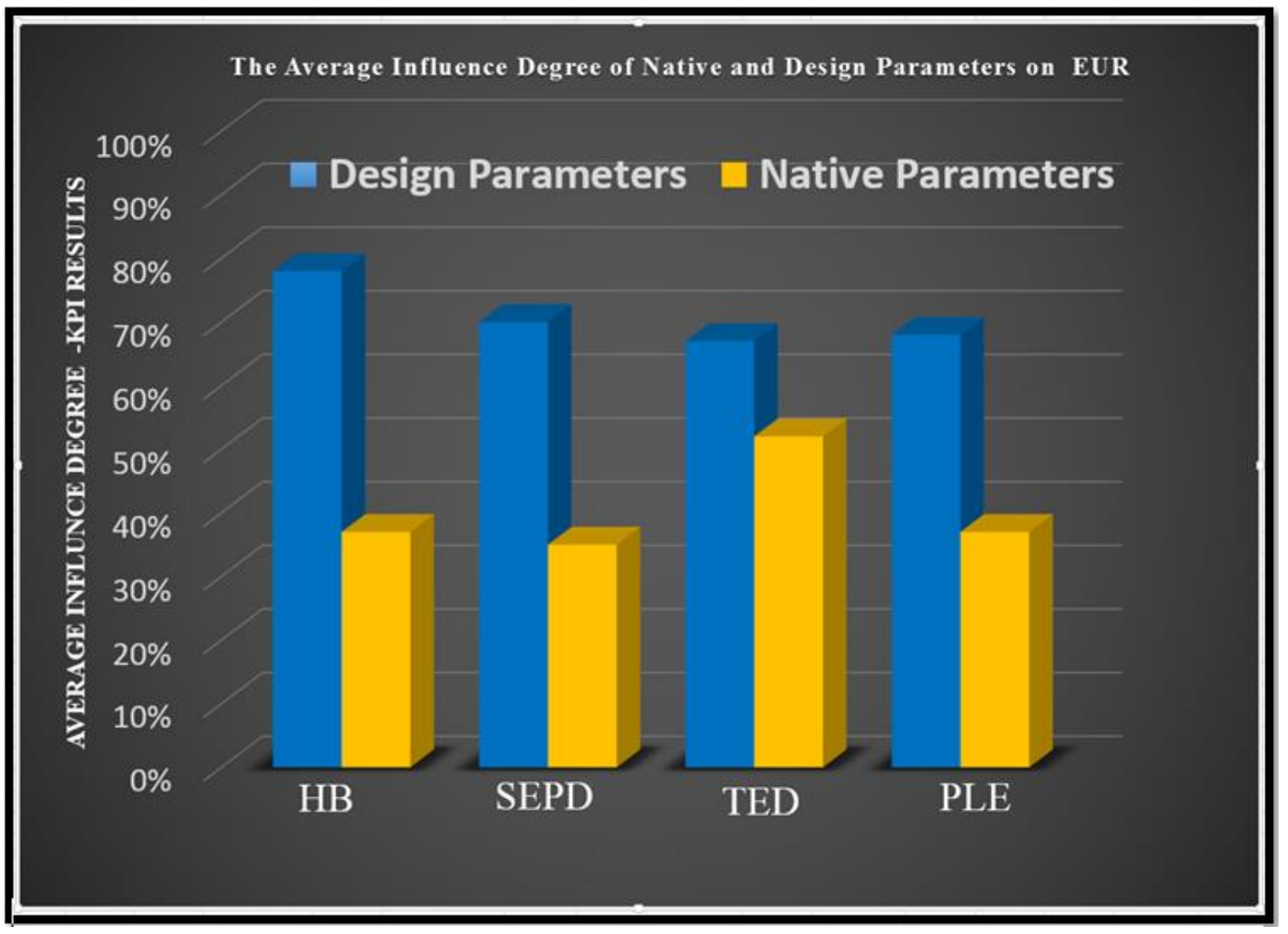

Figure 41: The Average Influence Degree of Native and Design Parameters on EUR 
2) Both Fuzzy Trend Analysis and Well Quality Analysis which are branches of the Fuzzy Pattern Recognition, successfully extract useful relationships between EUR and both reservoir and completion parameters. These relationships can be used as guidelines when developing an exploration project for shale reservoirs and furthermore when designing hydraulic fracture completion.

3) As expected, Total Organic Carbon was an effective indicator of productivity of the reservoirs and consequently had an impact on EUR. Interestingly, the ultimate calculated values for EUR were significantly different for each technique of Decline Curve analysis even though the same TOC percentage was used for all techniques.

4) The results of this study prove that the same general concepts which are applied to conventional reservoirs can also be applied to unconventional reservoirs such as tight gas shale. For example, when the formation exhibits high values of Young's Modulus, the EUR is lower than when the formation exhibits low values of Young's Modulus. In addition, EUR can be positively impacted by lateral length, proppant per stage and the total number of stages within a completion.

5) In order to maximize the ultimate EUR, decreased spacing between clusters should be used in horizontal wells. In general, the thickness and the matrix porosity have a positive impact on EUR. The Fuzzy Pattern Recognition successfully found these relations in shale gas reservoirs after unsuccessful attempts of using Conventional Statistical Analysis.

6) The findings of this study suggest that commencing production should begin with minimal delay after completion to improve the future production of the well.

7) Interestingly, this study shows that TED, a traditional method, is able to provide as reasonable of results when applied to a shale reservoir as when applied to a conventional reservoir.

8) The Artificial Neural Networks Models that built in this research can be used as guidelines when developing an exploration project for shale reservoirs and furthermore when designing hydraulic fracture completion. 
9) In addition, these Artificial Neural Networks Models can be used to predict EUR for wells that have not been drilled yet in this field.

10) IMPORTANT NOTE: Results shown in this study are highly case specific and should not be expected to have similar behavior when applied to another shale (or even a different asset in the same shale play).

\subsection{Recommendations for future works}

- Develop computer model based on the results of this study that has the ability to provide EUR for each single well by supplying the design and native parameters.

- It is recommended to study the impact of the native and the design parameters on EUR for other fields. 


\section{References}

1. Kanfar, Mohammed Sami A. "Comparison of Empirical Decline Curve Analysis for Shale Wells." PhD diss., Texas A\&M University, 2013.

2. Can, Bunyamin .Shah Kabir. "Probabilistic Production Forecasting for Unconventional Reservoirs With Stretched Exponential Production Decline Model." Ms. Thesis, Texas A\&M University, 2011.

3. Maley, S. "The Use of Conventional Decline Curve Analysis in Tight Gas Well Applications." SPE/DOE Low Permeability Gas Reservoirs Symposium. Society of Petroleum Engineers, 1985.

4. Cipolla, Craig L., Elyezer P. Lolon, Jim C. Erdle, and Barry Rubin. "Reservoir modeling in shale-gas reservoirs." SPE reservoir evaluation \& engineering 13, no. 04 (2010): 638653.

5. Ilk, Dilhan, Creties David Jenkins, and Thomas Alwin Blasingame. "Production Analysis in Unconventional Reservoirs-Diagnostics, Challenges, and Methodologies." In North American Unconventional Gas Conference and Exhibition. Society of Petroleum Engineers, 2011.

6. Duong, Anh N. "Rate-decline analysis for fracture-dominated shale reservoirs."SPE Reservoir Evaluation \& Engineering 14, no. 03 (2011): 377-387.

7. Ali, Tariq, James Sheng, and Mohamed Soliman. "New Production-Decline Models for Fractured Tight and Shale Reservoirs." In SPE Western North American and Rocky Mountain Joint Meeting. Society of Petroleum Engineers, 2014.

8. Valentine, Anne P., Alan Brown, Siddhartha Gupta, and Priyank Dwivedi. "Production Forecasting in Shales: A Comparative Field Data Study Using Large Well Counts." In SPE/CSUR Unconventional Resources Conference-Canada. Society of Petroleum Engineers, 2014.

9. McNeil, R., O. Jeje, and A. Renaud. "Application of the power law loss-ratio method of decline analysis." In Canadian International Petroleum Conference. Petroleum Society of Canada, 2009.

10. Mohaghegh, Shahab D. "Formation vs. Completion: Determining the Main Drivers Behind Production From Shale-A Case Study Using Data-Driven Analytics." Unconventional Resources Technology Conference (URTEC), 2015. 
11. Valkó, Peter P. "Assigning Value to Stimulation in the Barnett Shale: A Simultaneous Analysis of 7000 plus Production Histories and Well Completion Records." In SPE Hydraulic Fracturing Technology Conference. Society of Petroleum Engineers, 2009.

12. Ilk, Dilhan, Jay Alan Rushing, and Thomas Alwin Blasingame. "Decline-Curve Analysis for HP/HT Gas Wells: Theory and Applications." In SPE Annual Technical Conference and Exhibition. Society of Petroleum Engineers, 2009.

13. Valkó, Peter P., and W. John Lee. "A better way to forecast production from unconventional gas wells." In SPE Annual Technical Conference and Exhibition. Society of Petroleum Engineers, 2010.

14. Duong, Anh N. "An Unconventional Rate Decline Approach for Tight and FractureDominated Gas Wells." In Canadian Unconventional Resources and International Petroleum Conference. Society of Petroleum Engineers, 2010.

15. Freeborn, Randy, and Boyd Russell. "How to Apply Stretched Exponential Equations to Reserve Evaluation." In SPE Hydrocarbon Economics and Evaluation Symposium. Society of Petroleum Engineers, 2012.

16. Currie, Stephanie Marie, Dilhan Ilk, and Tom Blasingame. "Continuous estimation of ultimate recovery." In SPE Unconventional Gas Conference. Society of Petroleum Engineers, 2010.

17. Ilk, Dilhan, Stephanie Marie Currie, Dave Symmons, Jay Alan Rushing, and Thomas Alwin Blasingame. "Hybrid Rate-Decline Models for the Analysis of Production Performance in Unconventional Reservoirs." In SPE Annual Technical Conference and Exhibition. Society of Petroleum Engineers, 2010.

18. Towler, Brian F., Fundamental Principles of Reservoir Engineering, (Richardson, Texas, USA, 2002) Volume8, 176

19. Shahamat, Mohammad Sadeq, and Roberto Aguilera. "A new method for production decline analysis of tight gas formations." In Canadian Unconventional Resources and International Petroleum Conference. Society of Petroleum Engineers, 2010.

20. Mishra, Srikanta. "A New Approach to Reserves Estimation in Shale Gas Reservoirs Using Multiple Decline Curve Analysis Models." In SPE Eastern Regional Meeting. Society of Petroleum Engineers, 2012.

21. Dutta, Riteja, Marie Meyet, Chris Burns, and Frederik Van Cauter. "Comparison of empirical and analytical methods for production forecasting in unconventional reservoirs: 
Lessons from North America." In SPE/EAGE European Unconventional Resources Conference and Exhibition. 2014.

22. Esmaili, S., S. D. Mohaghegh, and A. Kalantari-Dahaghi. "Which Parameters Control Production in Shale Assets? A Pattern Recognition Study." (2013).

23. Esmaili, S., and S. D. Mohaghegh. "Using Data-Driven Analytics to Assess the Impact of Design Parameters on Production from Shale." In SPE Annual Technical Conference and Exhibition. Society of Petroleum Engineers, 2013.

24. Haghighat, S. Alireza, Shahab D. Mohaghegh, Vida Gholami, and David Moreno. "Production Analysis of a Niobrara Field Using Intelligent Top-Down Modeling." In SPE Western North American and Rocky Mountain Joint Meeting. Society of Petroleum Engineers, 2014.

25. Esmaili, S., S. D. Mohaghegh, and A. Kalantari-Dahaghi. "Shale Asset Production Evaluation by Using Pattern Recognition." In SPE Western Regional Meeting. Society of Petroleum Engineers, 2015.

26. Mohaghegh, Shahab D. "Formation vs. Completion: Determining the Main Drivers Behind Production From Shale-A Case Study Using Data-Driven Analytics." Unconventional Resources Technology Conference (URTEC), 2015.

27. Mohaghegh, S., and S. Ameri. "Artificial neural network as a valuable tool for petroleum engineers." Paper SPE 29220 (1995).

28. Garcia, Anangela, and Shahab D. Mohaghegh. "Forecasting US Natural Gas Production into year 2020: a comparative study." In SPE Eastern Regional Meeting. Society of Petroleum Engineers, 2004.

29. Kalantari-Dahaghi, Amirmasoud, Shahab Mohaghegh, and Soodabeh Esmaili. "Coupling numerical simulation and machine learning to model shale gas production at different time resolutions." Journal of Natural Gas Science and Engineering 25 (2015): 380-392.

30. Can, Bunyamin, and Shah Kabir. "Probabilistic Production Forecasting for Unconventional Reservoirs with Stretched Exponential Production Decline Model." SPE Reservoir Evaluation \& Engineering 15, no. 01 (2012): 41-50.

31. Mohaghegh, Shahab, Scott Reeves, and David Hill. "Development of an intelligent systems approach for restimulation candidate selection." In SPE/CERI Gas Technology Symposium. Society of Petroleum Engineers, 2000. 
32. Fetkovich, M. J. "Decline curve analysis using type curves." Journal of Petroleum Technology 32, no. 06 (1980): 1-065.

33. Javadi, Faegheh. "Estimating Ultimate Recovery in Shale Wells Based on." M.Sc. thesis. West Virginia University, 2014.

34. Gershenson, Carlos. "Artificial neural networks for beginners." arXiv preprint cs/0308031 (2003).

35. T BENEDICT, J. O. N. "The Mathematics of Decline Curves."

36. Huo, Da, Li Li, Xiaodong Fan, Yanmin Yu, and Kewen Li. "Decline curve analysis of oil production in low permeability reservoirs with great heterogeneity." In SPE Asia Pacific Oil and Gas Conference and Exhibition. Society of Petroleum Engineers, 2008.

37. Seshadri, Jagan Nathan, and Louis Mattar. "Comparison of power law and modified hyperbolic decline methods." In Canadian Unconventional Resources and International Petroleum Conference. Society of Petroleum Engineers, 2010.

38. Can, Bunyamin, and Shah Kabir. "Probabilistic Production Forecasting for Unconventional Reservoirs with Stretched Exponential Production Decline Model." SPE Reservoir Evaluation \& Engineering 15, no. 01 (2012): 41-50. 


\section{Appendix}

\section{Well Location (Easting)}

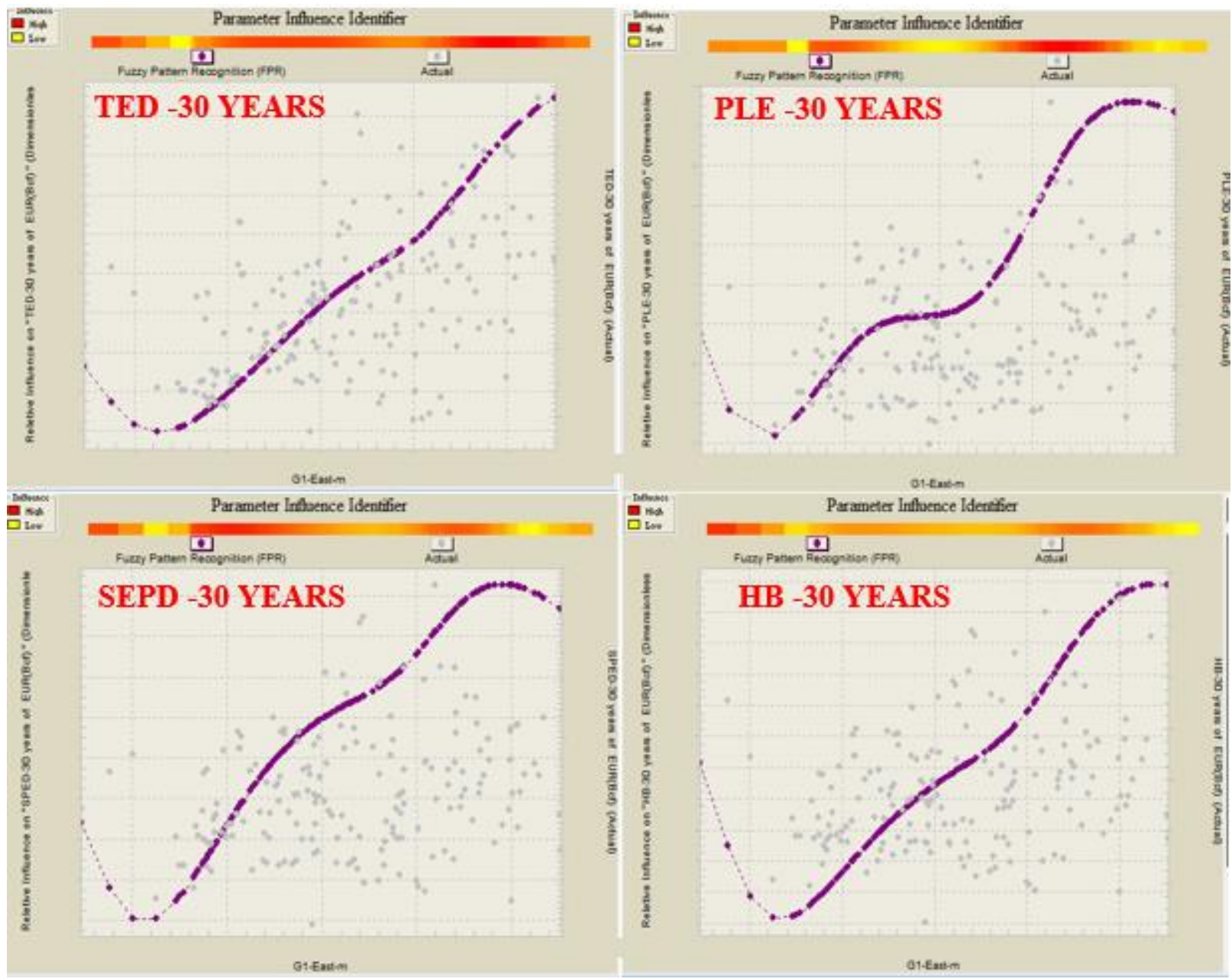

Figure 42: The impact of well location (Easting) on EUR for the models TED, PLE, SEPD and HB- FTA result 


\section{Poisson's Ratio}

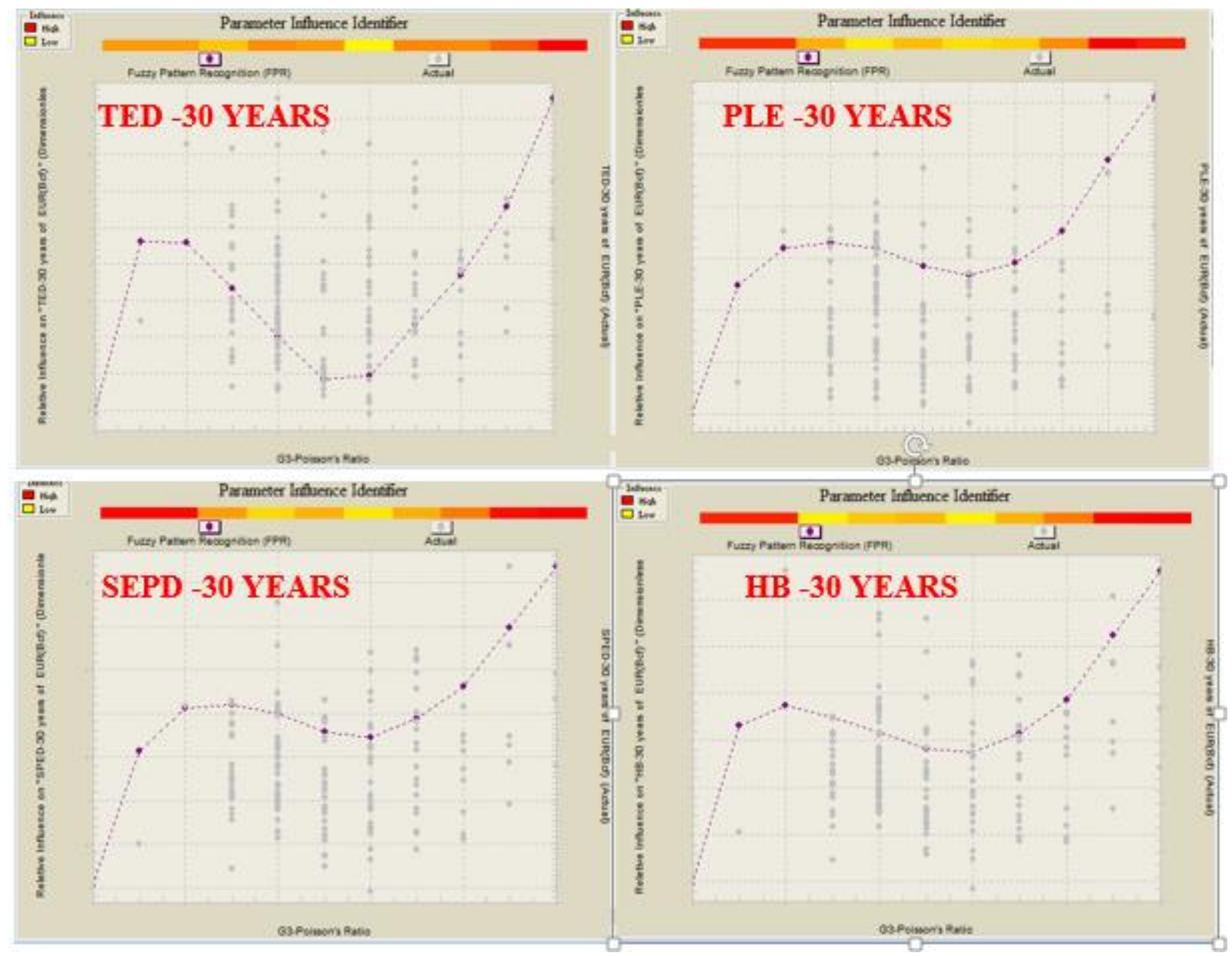

Figure 43: The impact of Poisson's Ratio on EUR for the models TED, PLE, SEPD and HBFTA result 


\section{Total Organic Carbon (Well Quality Analysis)}

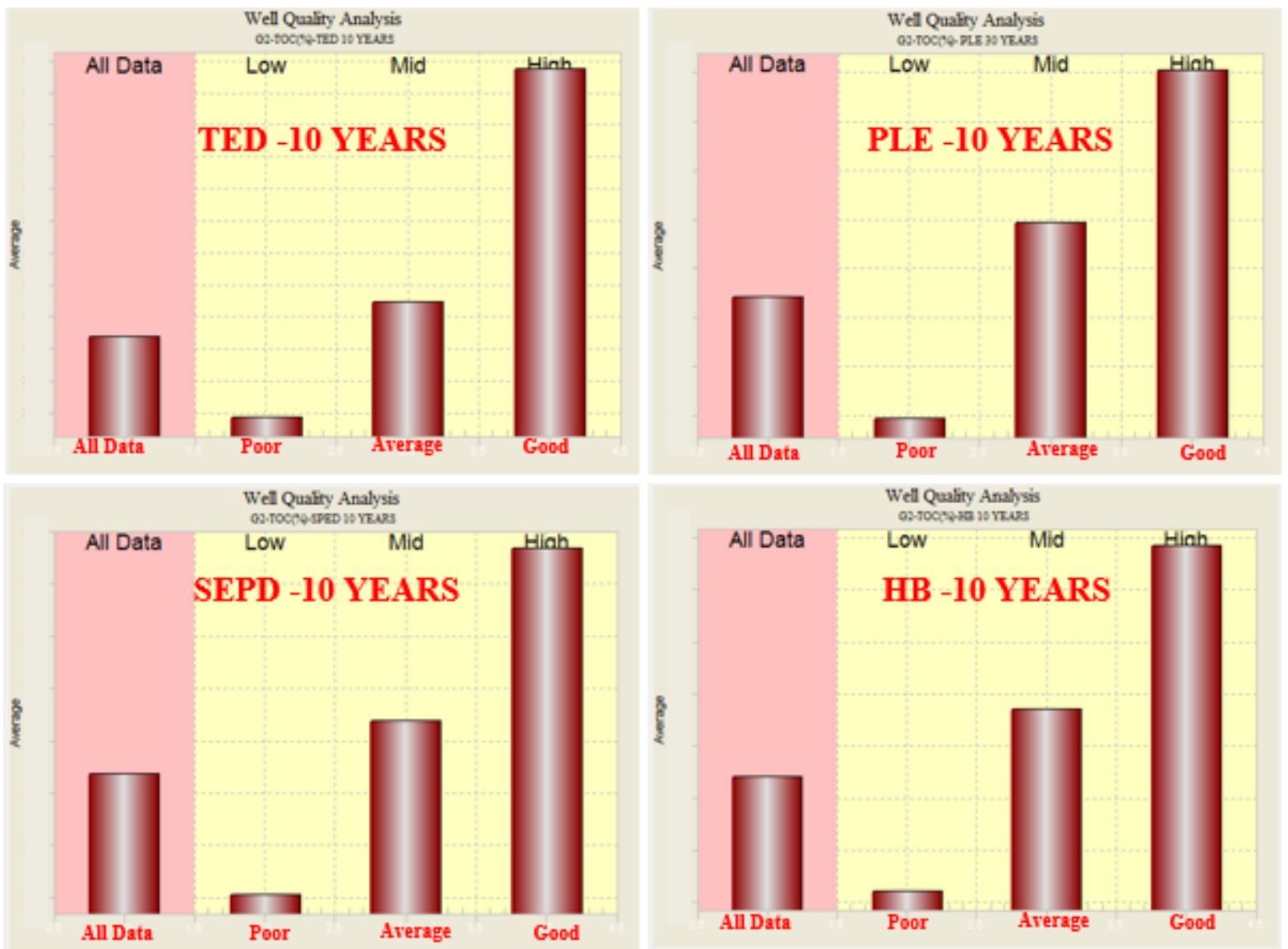

Figure 44: Well Quality Analysis of Total Organic Carbon for EUR models TED, PLE, SEPD and $\mathrm{HB}$ 


\section{Young's Modulus (Well Quality Analysis)}
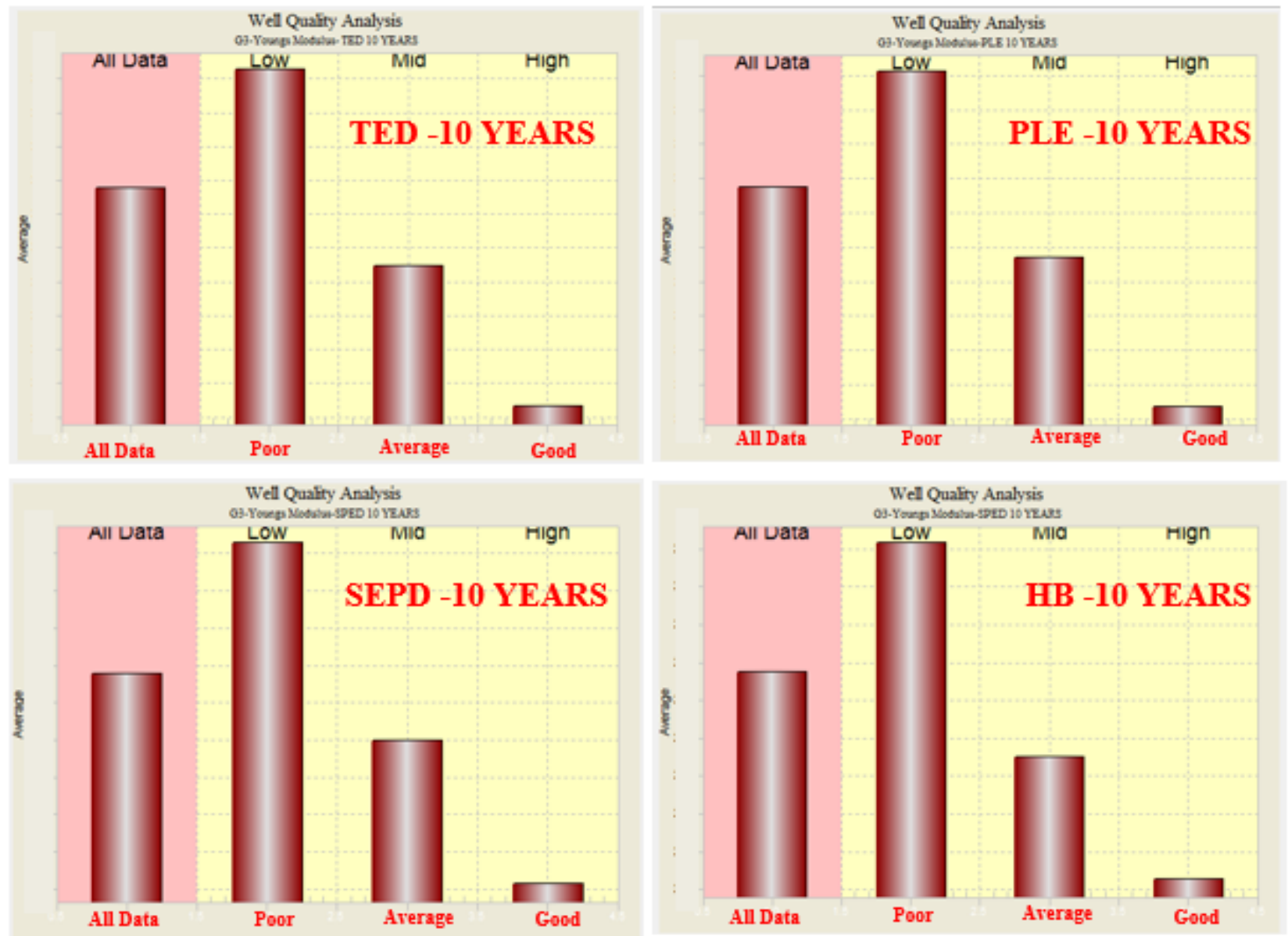

Figure 45: Well Quality Analysis of Young's Modulus for EUR models TED, PLE, SEPD and $\mathrm{HB}$ 


\section{Stimulated Lateral Length (Well Quality Analysis)}

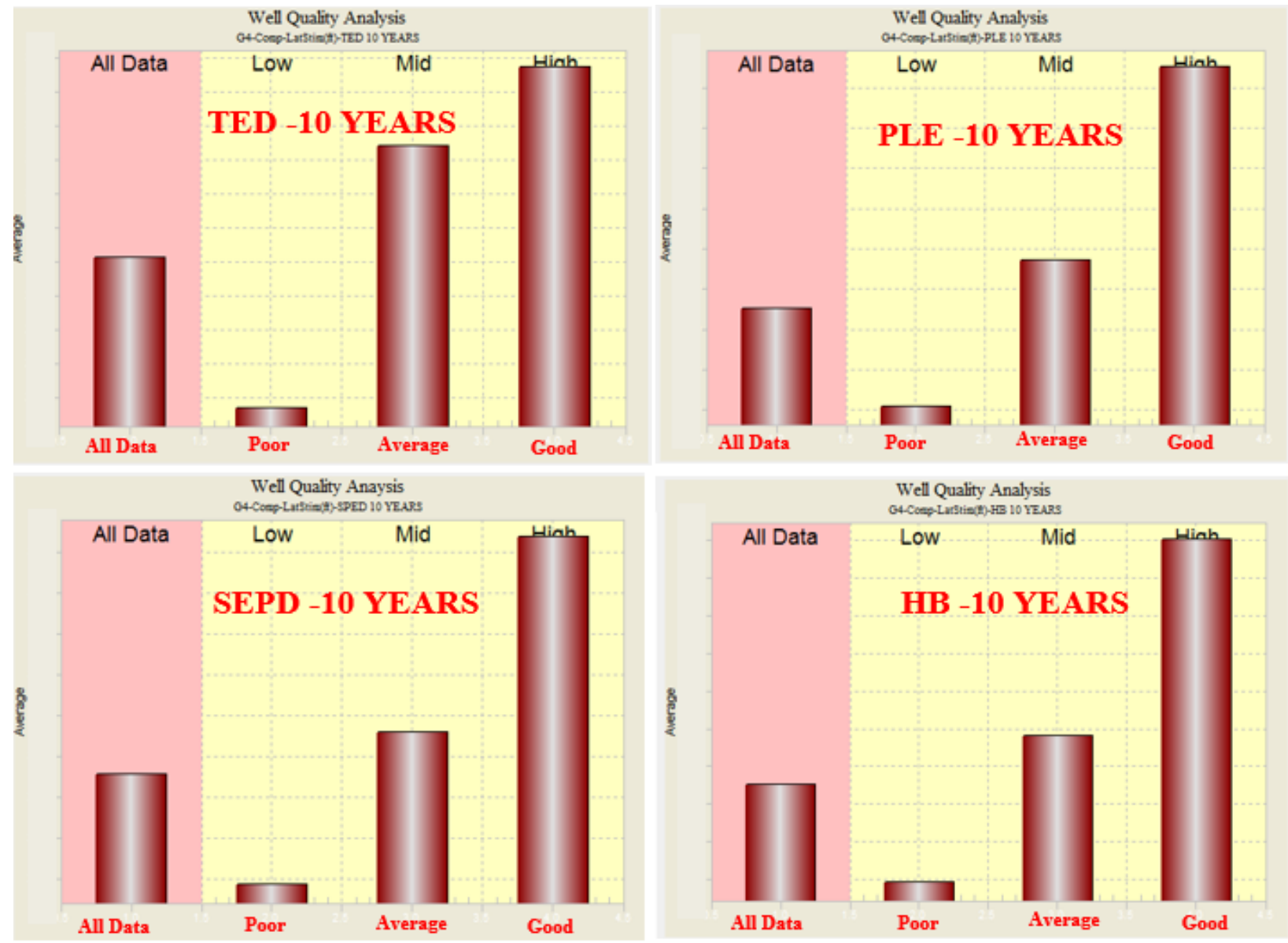

Figure 46: Well Quality Analysis of Lateral Length for EUR models TED, PLE, SEPD and HB 


\section{Number of Stages (Well Quality Analysis)}
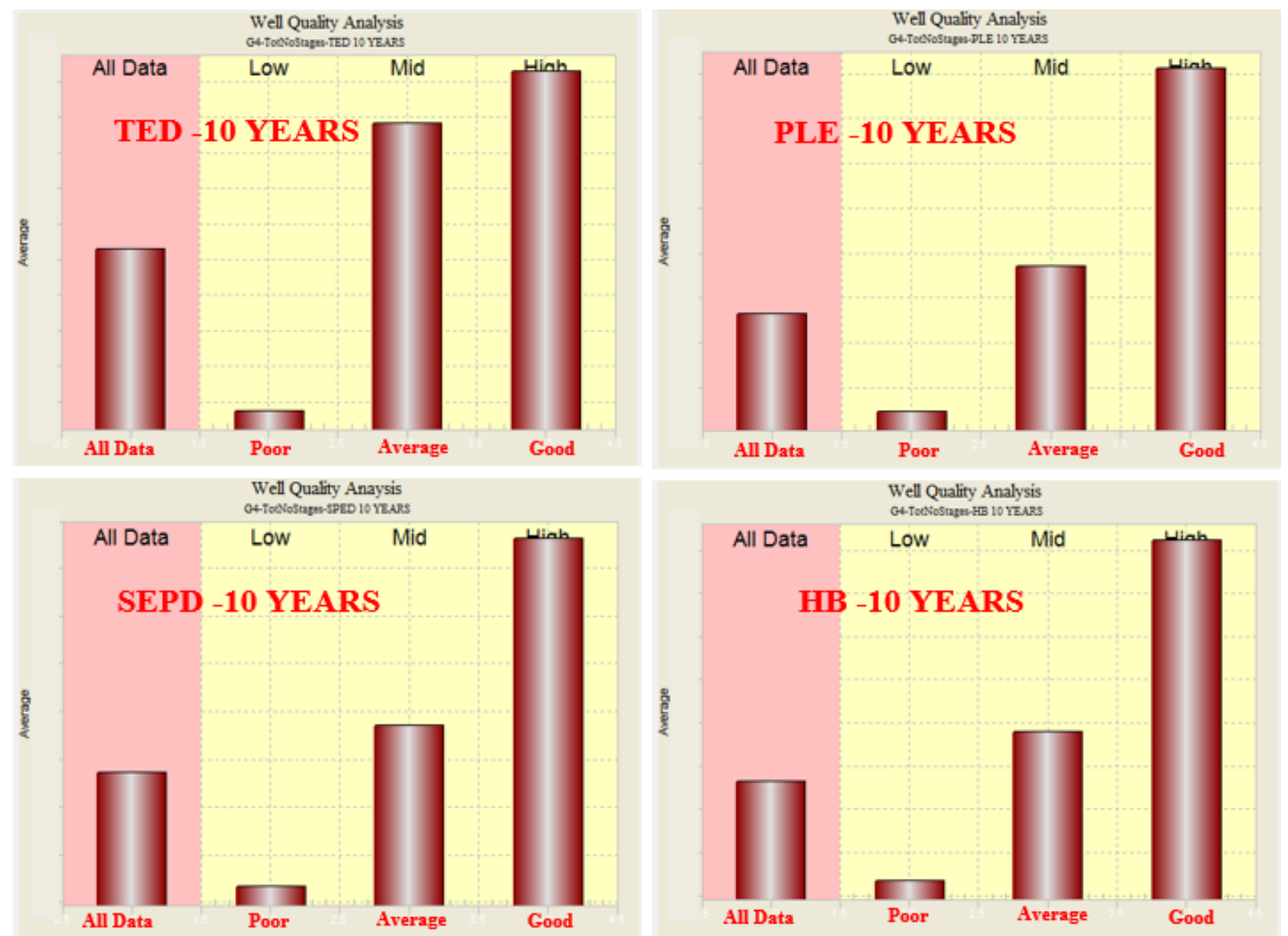

Figure 47: Well Quality Analysis of Number of Stages for EUR models TED, PLE, SEPD and $\mathrm{HB}$ 


\section{Injected Clean Volume per Stage}

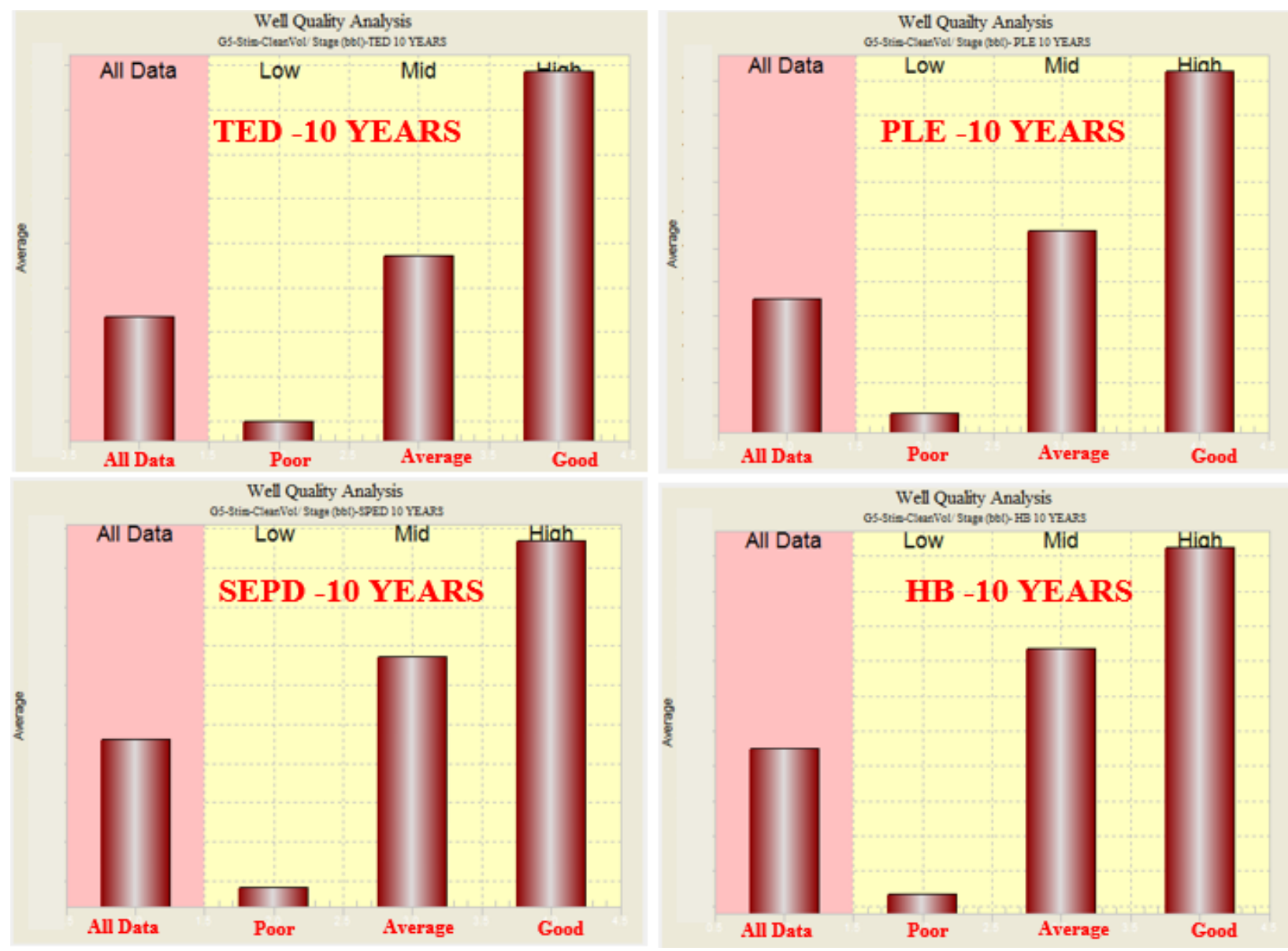

Figure 48: Well Quality Analysis of Injected Clean Volume per Stage for EUR models TED, PLE, SEPD and HB 


\section{EUR as a Function of Lateral Length and Cluster Spacing in Type Curve:}
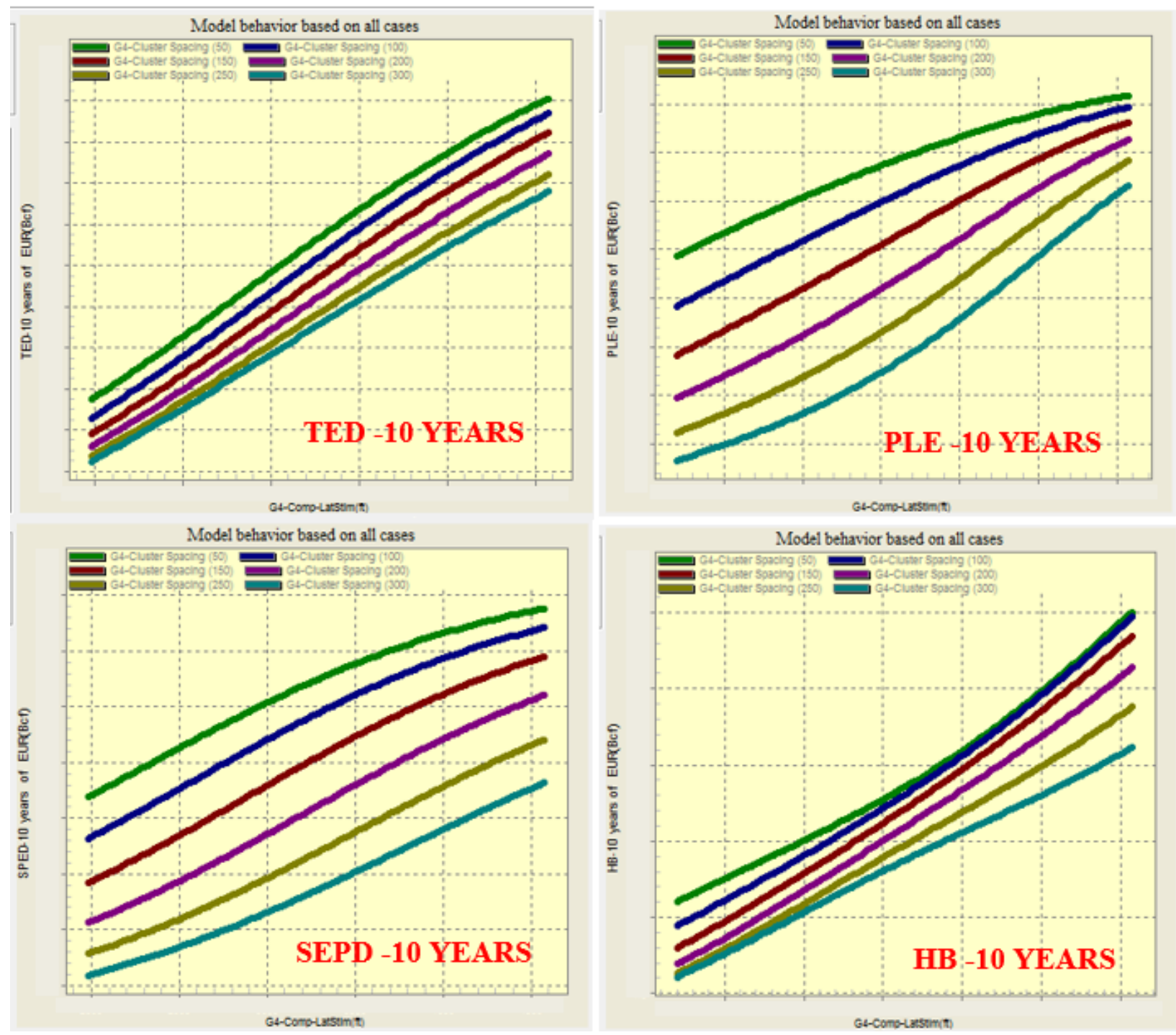

Figure 49: EUR as a Function of Lateral Length and Cluster Spacing in Type Curve 


\section{EUR as a Function of Lateral Length and Clean Volume in Type Curve:}
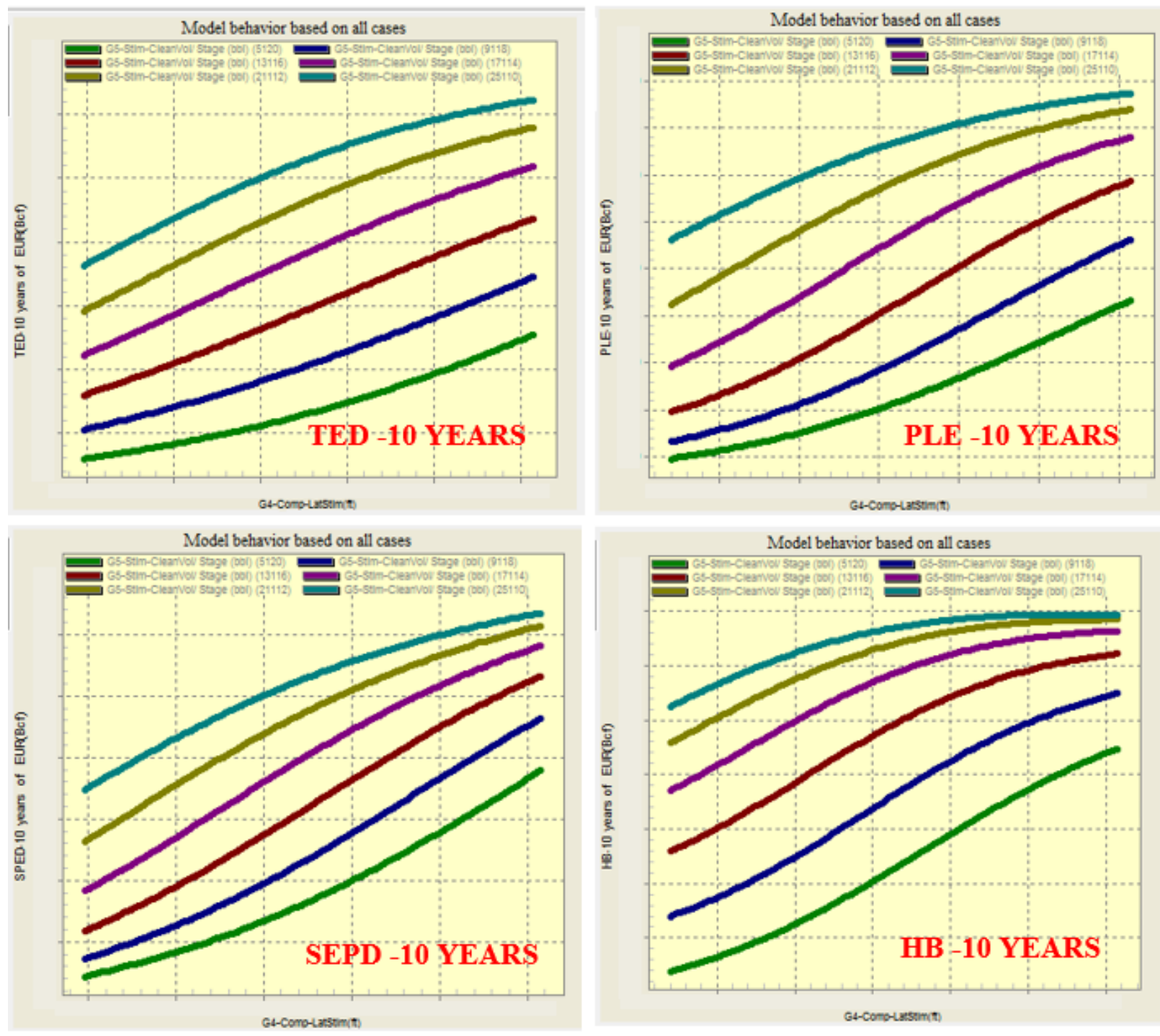

Figure 50: EUR as a Function of Lateral Length and Clean Volume in Type Curve 


\section{EUR as a Function of Lateral Length and Net Thickness in Type Curve:}
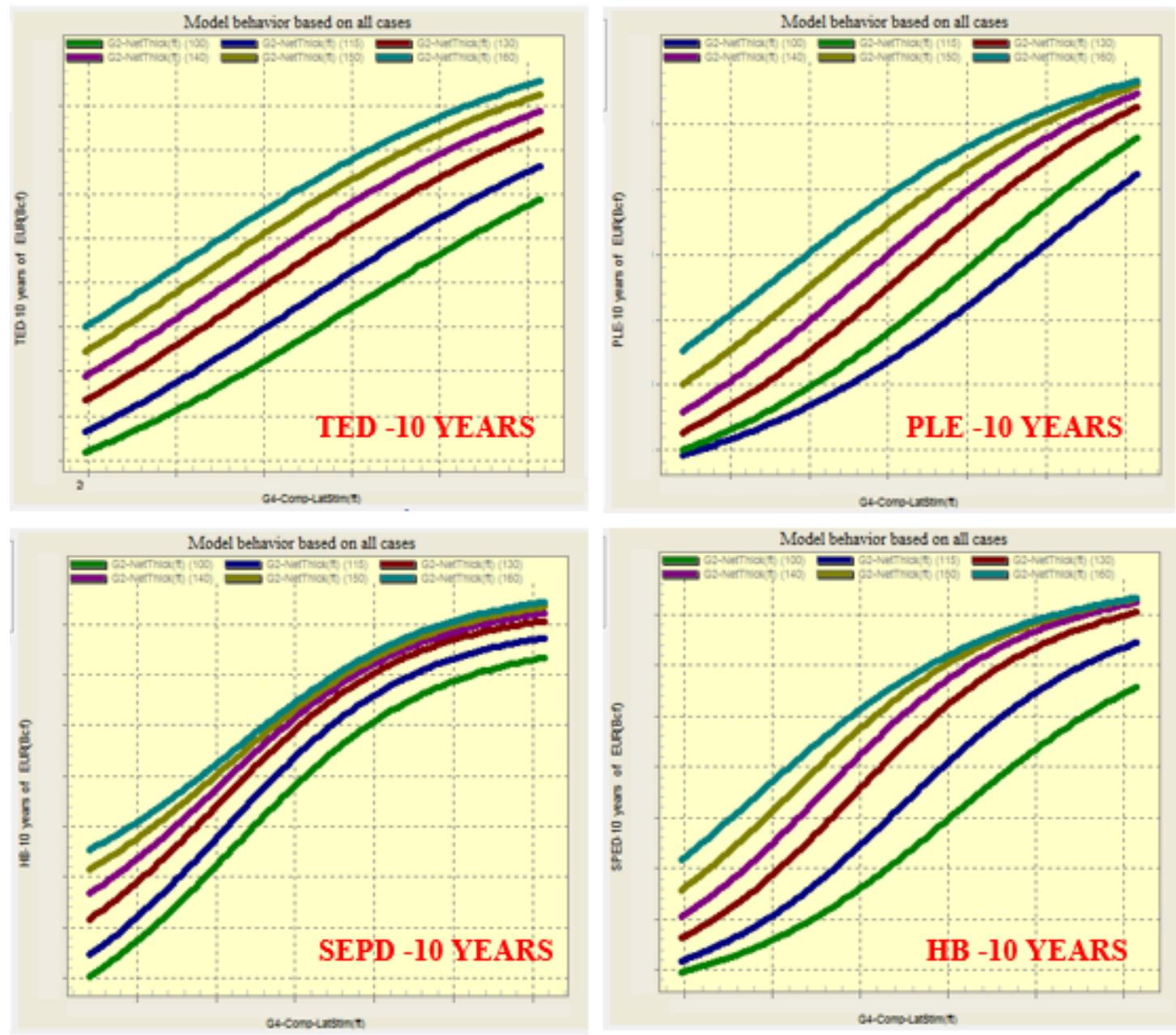

Figure 51: EUR as a Function of Lateral Length and Net Thickness in Type Curve 